\title{
The Dynamics of Polarized Beliefs in Networks Governed by Viral Diffusion and Media Influence
}

\author{
by
}

Mohammad Reza Sanatkar

Department of Electrical and Computer Engineering

Duke University

Date:

Approved:

Henry Pfister, Supervisor

Guillermo Sapiro

\begin{tabular}{c} 
Galen Reeves \\
\hline
\end{tabular}

Stacy Lynne Tantum

Patrick Charbonneau

Dissertation submitted in partial fulfillment of the requirements for the degree of Doctor of Philosophy in the Department of Electrical and Computer Engineering in the Graduate School of Duke University

2016 


\title{
$\underline{\text { ABSTRACT }}$ \\ The Dynamics of Polarized Beliefs in Networks Governed by Viral Diffusion and Media Influence
}

by

\author{
Mohammad Reza Sanatkar \\ Department of Electrical and Computer Engineering \\ Duke University
}

Date:

Approved:

\begin{tabular}{c}
\hline Henry Pfister, Supervisor \\
\hline Guillermo Sapiro \\
\hline Galen Reeves \\
\hline Stacy Lynne Tantum \\
\hline Patrick Charbonneau
\end{tabular}

An abstract of a dissertation submitted in partial fulfillment of the requirements for the degree of Doctor of Philosophy in the Department of Electrical and Computer Engineering

in the Graduate School of Duke University

2016 
Copyright (c) 2016 by Mohammad Reza Sanatkar All rights reserved except the rights granted by the Creative Commons Attribution-Noncommercial Licence 


\section{Abstract}

The multidimensional joint distributions that represent complex systems with many interacting elements can be computationally expensive to characterize. Methods to overcome this problem have been introduced by a variety of scientific communities. Here, we employ methods from statistics, information theory and statistical physics to investigate some approximation techniques for inference over factor graphs of spatially-coupled low density parity check (SC-LDPC) codes, estimation of the marginals of stationary distribution in influence networks consisting of a number of individuals with polarized beliefs, and estimation of per-node marginalized distribution for an adoption model of polarized beliefs represented by a Hamiltonian energy function.

The second chapter introduces a new method to compensate for the rate loss of SC-LDPC codes with small chain lengths. Our interest in this problem is motivated by the theoretical question of whether or not the rate loss can be eliminated by small modifications to the boundary of the protograph? We tackle this question by attaching additional variable nodes to the check nodes at the chain boundary. Our

goal is to increase the code rate while preserving the BP threshold of the original chain.

In the third chapter, we consider the diffusion of polarized beliefs in a social network based on the influence of neighbors and the effect of mass media. The adoption process is modeled by a stochastic process called the individual-based (IN-STOCH) 
system and the effects of viral diffusion and media influence are treated at the individual level. The primary difference between our model and other recent studies, which model both interpersonal and media influence, is that we consider a third state, called the negative state, to represent those individuals who hold positions against the innovation in addition to the two standard states neutral (susceptible) and positive (adoption). Also, using a mean-field analysis, we approximate the IN-STOCH system in the large population limit by deterministic differential equations which we call the homogeneous mean-field (HOM-MEAN) and the heterogeneous mean-field (HET-MEAN) systems for exponential and scale-free networks, respectively. Based on the stability of equilibrium points of these dynamical systems, we derive conditions for local and global convergence, of the fraction of negative individuals, to zero.

The fourth chapter also focuses on the diffusion of polarized beliefs but uses a different mathematical model for the diffusion of beliefs. In particular, the Potts model from statistical physics is used to model the joint distribution of the individual's states based on a Hamiltonian energy function. Although the stochastic dynamics of this model are not completely defined by the energy function, one can choose any Monte Carlo sampling algorithm (e.g., Metropolis-Hastings) to define Markov-chain dynamics. We are primarily interested in the stationary distribution of the Markov chain, which is given by the Boltzmann distribution. The fraction of individuals in each state at equilibrium can be estimated using both Markov-chain Monte Carlo methods and the belief-propagation (BP) algorithm. The main benefit of the Potts model is that the BP estimates are asymptotically exact in this case. 


\section{Contents}

Abstract iv

List of Tables viii

List of Figures $\quad$ ix

1 Introduction 1

1.1 Increasing the Rate of Spatially-Coupled Codes via Optimized Irregular Termination . . . . . . . . . . . . . . . . . 1

1.2 The Dynamics of Polarized Beliefs in Networks Governed by Viral Diffusion and Media Influence . . . . . . . . . . . . . . . 2

1.3 Modeling the Dynamics of Polarized Beliefs in Networks Governed by Viral Diffusion and Media Influence Using the Potts Model . . . . . . 4

2 Increasing the Rate of Spatially-Coupled Codes via Optimized Irregular Termination $\quad 6$

2.1 Introduction $\ldots \ldots \ldots \ldots \ldots \ldots \ldots \ldots \ldots \ldots \ldots \ldots \ldots \ldots \ldots$

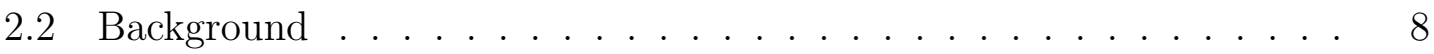

2.3 Code Design . . . . . . . . . . . . . . . . . . . . . . . 10

2.4 Density Evolution Equations . . . . . . . . . . . . . . . . . 11

2.5 Degree Distribution Optimization . . . . . . . . . . . . . . . 14

2.5.1 Equivalent LP formulation . . . . . . . . . . . 15

2.6 Results and Discussion . . . . . . . . . . . . . . . . . . 18

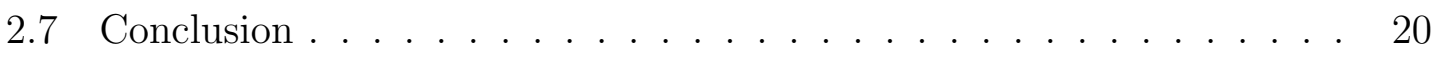


3 The Dynamics of Polarized Beliefs in Networks Governed by Viral Diffusion and Media Influence $\quad 22$

3.1 Introduction . . . . . . . . . . . . . . . . . . . . . . 22

3.2 Individual-Based Stochastic System . . . . . . . . . . . . . . . 28

3.3 Exponential Networks $\ldots \ldots \ldots$. . . . . . . . . . . . . . . 31

3.3 .1 Equilibrium Points . . . . . . . . . . . . . . . 33

3.3.2 Stability of Equilibrium Points . . . . . . . . . . . . . . 34

3.4 Scale-Free Networks . . . . . . . . . . . . . . . . . . . . . . . . 43

3.4.1 Barabasi-Albert Networks . . . . . . . . . . . . . . . . . 47

3.5 Simulation Results . . . . . . . . . . . . . . . . . 50

3.6 Conclusion . . . . . . . . . . . . . . . . . . . . . 57

4 Modeling the Dynamics of Polarized Beliefs in Networks Governed by Viral Diffusion and Media Influence Using the Potts Model 60

4.1 Introduction $\ldots \ldots \ldots \ldots \ldots \ldots \ldots \ldots \ldots \ldots \ldots \ldots \ldots \ldots$

4.2 Estimation of Node-Type Marginal Distributions using Belief Propa-

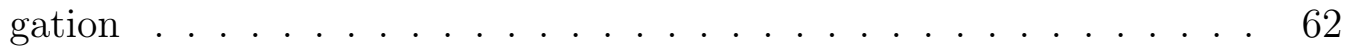

4.3 Belief Propagation Equations _. . . . . . . . . . . . . . . 63

4.4 Fixed-Point Belief-Propagation Equations . . . . . . . . . 65

4.5 Density Evolution for Erdős-Reńyi Random Graphs . . . . . . . . . 67

4.6 Results . . . . . . . . . . . . . . . . . . . . . . . . 69

4.7 A Comparison Between Different Scenarios . . . . . . . . . . . 72

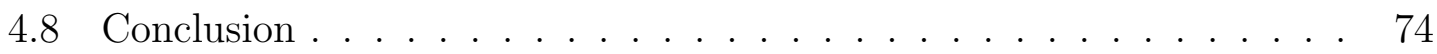

$\begin{array}{ll}\text { Bibliography } & 78\end{array}$

$\begin{array}{ll}\text { Biography } & 83\end{array}$ 


\section{List of Tables}

2.1 Code rates and the $\mathrm{BP}$ thresholds corresponding to constant node degree distributions for $(3,6,40)$ protograph. . . . . . . . . . . . . 19

2.2 Code rates and the BP thresholds corresponding to constant node degree distributions for $(5,10,40)$ protograph. . . . . . . . 20 


\section{List of Figures}

$2.1 \quad(3,6,5)$ spatially-coupled protograph. . . . . . . . . . . . . 9

2.2 Irregular variable nodes with degree distribution $\lambda(x)$ attached to the folded $(3,6,12) \mathrm{SC}$ chain. . . . . . . . . . . . . . . . 11

2.3 Irregular and spatially-coupled subsystems. . . . . . . . . . . . . . 15

$2.4 \delta(z)$ versus $z$ for the $(3,6,40)$ and $(5,10,40)$ ensembles. . . . . . . . 16

3.1 Flowchart of the individual-based stochastic system. A neutral node may become positive with a probability $\beta$ per its positive neighbors and because of media influence with a probability $\alpha$. A positive node may return to the neutral state with a probability $\gamma$. A neutral node may become negative with a probability $\mu$ per its negative neighbors. A negative node may return to the neutral state with a probability $\theta$ and to the positive state with a probability $\delta \alpha \ldots \ldots . . . . . . .28$

3.2 Final fraction of negative individuals for WS networks as a function of $\mu / \beta$. Solid lines are theoretical predictions. Dashed lines are simulation results. Parameter values: $\theta=0.01, \beta=0.001, \alpha=0.01$, $\delta=0.4$, and $\gamma=0.01 . \ldots \ldots \ldots \ldots$. . . . . . . . . 50

3.3 Final fraction of negative individuals for random regular networks as a function of $\mu / \beta$. Solid lines are theoretical predictions. Dashed lines are simulation results. Parameter values: $\theta=0.01, \beta=0.001$,

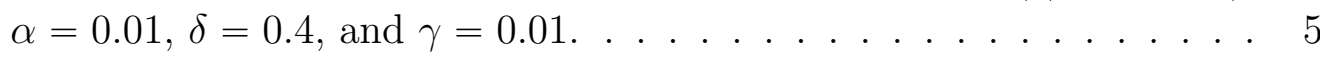

3.4 Comparison between viral diffusion and media influence over the WS network (Parameter values: $\theta=0.0001, \delta=0.05, \gamma=0.5$, and

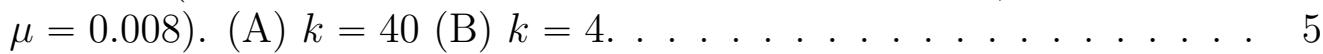

3.5 Final fraction of negative individuals for BA networks as a function of $\mu / \beta$. Solid lines are theoretical predictions. Dashed lines are simulation results. Parameter values: $\theta=0.01, \beta=0.001, \alpha=0.01$, $\delta=0.4$, and $\gamma=0.01 \ldots \ldots \ldots \ldots$. . . . . . . . . . . 54 
3.6 Histogram of node degrees of the ego-Facebook network. . . . . . . . 55

3.7 Final fraction of negative individuals in the ego-Facebook network as a function of $\mu / \beta$. The solid line is the theoretical predictions. The dashed line is the simulation results. Parameter values: $\theta=0.01$, $\beta=0.001, \alpha=0.01, \delta=0.4$, and $\gamma=0.01 \ldots \ldots . . . . . .$.

4.1 Subgraph with depth 1 of the factor graph of the proposed model for

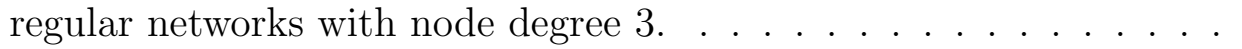

4.2 Node-type marginal of stationary distribution using the $\mathrm{MH}$ algorithm for regular networks with node degree $k=4$ as a function of $J_{N U} / J_{N P}$. Parameter values: $J_{N P}=1.0, J_{P U}=2.0, h_{N}=1.5, h_{P}=1.0, h_{U}=$ 1.0 , and $\beta=1.0 . \ldots \ldots \ldots \ldots$

4.3 The sample autocorrelation for the $\mathrm{MH}$ algorithm for regular networks with node degree $k=4$. Parameter values: $J_{N P}=1.0, J_{P U}=2.0$, $h_{N}=1.5, h_{P}=1.0, h_{U}=1.0$, and $\beta=1.0 \ldots \ldots . . . . .$.

4.4 Estimation of node-type marginal distribution using the BP algorithm for regular networks with node degree $k=4$ as a function of $J_{N U} / J_{N P}$. Parameter values: $J_{N P}=1.0, J_{P U}=2.0, h_{N}=1.5, h_{P}=1.0, h_{U}=$ 1.0 , and $\beta=1.0 . \ldots \ldots \ldots \ldots$

4.5 (left) Node-type marginal of stationary distribution using the $\mathrm{MH}$ algorithm and (right) node-type marginal of stationary distribution using the BP algorithm for Erdős-Reńyi random networks with 1000 nodes and $p=0.004$ as a function of $J_{N U} / J_{N P}$. Parameter values: $J_{N P}=1.0, J_{P U}=3.0, h_{N}=4.0, h_{P}=3.0, h_{U}=2.0$, and $\beta=1.0 .$.

4.6 Estimation of Node-type marginal of stationary distribution using the DE analysis of BP algorithm for Erdős-Reńyi random networks with 1000 nodes and $p=0.004$ as a function of $J_{N U} / J_{N P}$. Parameter values: $J_{N P}=1.0, J_{P U}=3.0, h_{N}=4.0, h_{P}=3.0, h_{U}=2.0$, and $\beta=1.0 . \quad$.

4.7 Estimation of node-type marginal distribution using the BP algorithm for regular networks with node degree $k=4$ (left) and $k=12$ (right) as a function of $J_{N U} / J_{N P}$. Parameter values: $J_{N P}=1.0, J_{P U}=2.0$, $h_{N}=1.5, h_{P}=1.0, h_{U}=1.0$, and $\beta=1.0 . \ldots \ldots \ldots$

4.8 Estimation of node-type marginal distribution using the BP algorithm for regular networks with node degree $k=36$ as a function of $J_{N U} / J_{N P}$. Parameter values: $J_{N P}=1.0, J_{P U}=2.0, h_{N}=1.5$, $h_{P}=1.0, h_{U}=1.0$, and $\beta=1.0 \ldots \ldots \ldots$. . . . . . . 76 
4.9 Estimation of node-type marginal distribution using the BP algorithm for regular networks with node degree $k=4$ (left) and $k=12$ (right) as a function of $J_{N U} / J_{N P}$. Parameter values: $J_{N P}=1.0, J_{P U}=1.0$, $h_{N}=1.5, h_{P}=1.0, h_{U}=1.0$, and $\beta=1.0 \ldots \ldots \ldots \ldots$

4.10 Estimation of node-type marginal distribution using the BP algorithm for regular networks with node degree $k=36$ as a function of $J_{N U} / J_{N P}$. Parameter values: $J_{N P}=1.0, J_{P U}=1.0, h_{N}=1.5$, $h_{P}=1.0, h_{U}=1.0$, and $\beta=1.0 \ldots \ldots \ldots \ldots$ 


\section{Introduction}

In this thesis, we consider inference problems from three different areas that are based on different mathematical models. They do share, however, a common property: An analytical closed-form solution for the distribution of interest cannot be computed efficiently. Throughout this thesis, we address issue with a variety of analytical and numerical methods borrowed from statistics, information theory, and statistical physics.

\subsection{Increasing the Rate of Spatially-Coupled Codes via Optimized Irregular Termination}

Low-density parity-check (LDPC) codes and the belief-propagation (BP) decoding were invented by Gallager in 1963 (Gallager (1963)). Luby et al. employed irregular graphs and optimized degree distributions to construct capacity-achieving LDPC codes for the binary erasure channel (BEC) (Luby et al. (1997)). Urbanke et al. introduced a method called density evolution (DE) to design long irregular LPDC codes that could approach capacity on binary-input AWGN channels (Richardson and Urbanke (2002)). Spatially-coupled LDPC (SC-LDPC) codes, also called convolutional LDPC codes, are the convolutional counterparts of LDPC block codes (Jiménez Fel- 
ström and Zigangirov (1999)). The SC-LDPC codes we consider are constructed by coupling a chain of regular LDPC Tanner graphs. Increasing the length of this chain decreases the rate-loss due to termination. For delay-sensitive applications, smaller chain lengths are preferred but this leads to an increased rate loss.

In Chapter 2, we propose a new method to compensate for the rate loss of SCLDPC codes with small chain lengths. Our interest in this problem is motivated by the theoretical question of whether or not the rate loss can be eliminated by small modifications to the boundary of the protograph? We tackle this question by attaching additional variable nodes to the check nodes at the chain boundary. Our goal is to increase the code rate while preserving the BP threshold of the original chain. The new variable nodes have degrees that are chosen from an irregular degree distribution. They connect to boundary check nodes using the edges that were previously removed by termination. Although we only consider a specific parameterized modification, our results suggest that a small modification to the boundary cannot make the rate loss negligible.

\subsection{The Dynamics of Polarized Beliefs in Networks Governed by Viral Diffusion and Media Influence}

To design effective marketing strategies that promote brand awareness, the adoption of innovations, or the popularity of new products, it is crucial to take into account the influence networks of targeted populations. In influence networks, nodes represent individuals and links describe influence relationships between them. The topology of an influence network specifies the underlying structure of influence relationships between individuals. Marketing strategies can be divided into the two broad categories: viral and media marketing. Viral marketing exploits the structures of influence networks to activate existing influence to target potential adopters, and form global cascades of adopters in influence networks. In particular, viral market- 
ing is designed based on word-of-mouth and encourages individuals to share product information with their social contacts. Therefore, the medium for viral marketing is the connectivity of a social network. On the other hand, media marketing treats all individuals as atomized objects of global media influence without taking into account their social networks. Media marketing is a broadcast mechanism that acts externally on influence networks because all the individuals receive the media influence directly from the same source. TV and newspaper ads are examples of media marketing while personalized referrals and recommendations are examples of viral marketing. Studying the diffusion of new ideas, beliefs, and technologies, collectively called innovation, started about 120 years ago (Dearing (2008)).

Marketing campaigns can be seen as external change agents that interact with the dynamics of innovation in influence networks to maximize their primary output measures. These goal of marketing is to effect maximal change with minimal cost (Dearing (2008)). Diffusion of innovations, viral marketing, has been studied extensively. A myriad of studies show that the decision process of individuals is affected by mixtures of interpersonal and media influence (Watts and Dodds (2007)). Analyzing empirical diffusion patterns over seven different online domains, Goel et al. concluded that these diffusion patterns motivate models that explicitly take into account media marketing in addition to viral marketing (Goel et al. (2012)). While most studies of the diffusion process assume person-to-person networks as the only medium for diffusion of innovations, a number of recent studies consider both global influence by external sources and interpersonal networks as mechanisms of diffusion (Goel et al. (2016); Myers et al. (2012); Farajtabar et al. (2014); Kleineberg and Boguñá (2014)).

In Chapter 3, we propose a stochastic system to model adoption process of polarized beliefs governed by viral diffusion and media influence at the individual level. The primary difference between our model and other recent studies that model both 
interpersonal and media influence is the following: we consider a third state, called negative state, to represent those individuals who hold positions against the innovation in addition to two existing states (neutral (susceptible) and positive (adoption)) in (Goel et al. (2012, 2016); Myers et al. (2012); Farajtabar et al. (2014); Kleineberg and Boguñá (2014)). First, we propose a stochastic system to model the dynamics of polarized beliefs at the individual level. This stochastic system is a Markov chain and is called the individual-based stochastic (IN-STOCH) system. This system is described by a set of individual-based transition events that govern dynamics of polarized belief propagation in influence networks. Then, using a mean-field analysis, we approximate the IN-STOCH system in the large population limit by deterministic differential equations to derive the homogeneous mean-field (HOM-MEAN) and the heterogeneous mean-field (HET-MEAN) systems for exponential and scale-free networks, respectively. Based on the stability of equilibrium points of these dynamical systems, we derive conditions for local and global convergence, of the fraction of negative individuals, to zero. Critical values of model parameters corresponding to these conditions characterize different phase transitions of polarized dynamics.

\subsection{Modeling the Dynamics of Polarized Beliefs in Networks Gov- erned by Viral Diffusion and Media Influence Using the Potts Model}

The model derived in Chapter 3 to formulate the adoption of polarized beliefs considers the following transitions among the three states of neutral, positive and negative for every individual: viral and diffusion transitions from neutral to positive state; diffusion transition from positive to neutral state; viral transition from neutral to negative state; diffusion transition from negative to neutral state; diffusion transition from negative to positive state. In other words, out of 12 possible diffusion and viral transitions, this model considers 6 of them. In Chapter 4, we propose a new adoption 
model for polarized beliefs that allows all the 12 viral and diffusion transitions among the three states of neutral, positive and negative for every individual. In this model, the propagation of polarized beliefs is modeled using the Potts model borrowed from the statistical physics community (Potts (1952)).

Following the Potts model, we model the transitions of individuals by defining an Hamiltonian energy function. Since computing the partition function for the Boltzmann distribution requires exponential complexity, we use the Metropolis-Hastings (MH) algorithm to estimate the marginalized stationary per node distribution. We also employ the BP algorithm to analytically approximate the node-type marginal distribution. Finally, we show that the resulting distributions agree. In contrast to the model proposed in Chapter 3, analyzing the fixed points of the derived dynamical system based on ordinary differential equations, we can study an approximations of population measures of the original stochastic system. However, we are not able to analyze directly the IN-STOCH system. For the proposed model in Chapter 4, we can analyze the BP algorithm for regular networks to compute the node-type marginal of stationary distribution. 


\section{2}

\section{Increasing the Rate of Spatially-Coupled Codes via Optimized Irregular Termination}

In this chapter, we consider the rate-loss problem for spatially-coupled LDPC (SCLDPC) codes on the binary erasure channel. Although SC-LDPC codes have good noise thresholds under belief-propagation (BP) decoding, they also suffer a rate-loss due to termination that is significant at moderate blocklengths. Our idea is to attach additional variable nodes at the boundary using an irregular degree distribution. Then, this degree distribution is optimized to improve the code rate without reducing the BP threshold. The optimization is formulated as an linear program and solved numerically. Our results show that the code rate can be increased by a reasonable amount without decreasing the BP threshold.

\subsection{Introduction}

Low-density parity-check (LDPC) codes and iterative decoding were invented by Gallager in 1963 (Gallager (1963)). LDPC codes are linear codes defined by a sparse parity-check matrix that is also viewed as the adjacency matrix of a sparse bipartite 
Tanner graph. The key feature that distinguishes LDPC codes from earlier approaches is the efficiency of the belief propagation (BP) decoding algorithm, which works by passing messages along the edges of the sparse bipartite graph. This allows the construction and decoding of very long codes (Jr. et al. (2013)).

LDPC codes were largely forgotten for more than 30 years after their discovery. Then, they were rediscovered independently by two groups (Sipser and Spielman (1996); MacKay and Neal (1996)). Then, Luby et al. employed irregular graphs and optimized degree distributions to construct capacity-achieving LDPC codes for the binary erasure channel (BEC) (Luby et al. (1997)). Later, Urbanke et al. introduced a method called density evolution (DE) to design long irregular LPDC codes that could approach capacity on binary-input AWGN channels (Richardson and Urbanke (2002)). In particular, for any binary-input memoryless symmetric (BMS) channel, this method can be used to precisely analyze the evolution of the messages on the edges of the graph during iterative decoding.

Spatially-coupled LDPC (SC-LDPC) codes, also called convolutional LDPC codes, are the convolutional counterparts of LDPC block codes (Jiménez Felström and Zigangirov (1999)). SC-LDPC codes combine the capacity-approaching iterative decoding thresholds of optimized irregular LDPC codes with the linear growth of minimum distance of regular LDPC codes to achieve good performance and lower error floors (Jr. et al. (2013)). The outstanding performance of SC-LDPC codes under BP decoding is explained by Kudekar, Richardson, and Urbanke as threshold saturation via spatial coupling where the $\mathrm{BP}$ decoding threshold approaches the maximum a posteriori (MAP) decoding threshold. Using this, regular SC-LDPC codes with BP decoding have been shown to approach capacity universally over the class of binary memoryless symmetric channels (Kudekar et al. (2011, 2012)).

The SC-LDPC codes we consider are constructed by coupling a chain of regular LDPC Tanner graphs. Increasing the length of this chain decreases the rate-loss due 
to termination. For delay-sensitive applications, smaller chain lengths are preferred but this leads to an increased rate loss. In (Tazoe et al. (2012)), it is proposed to modify chains by removing some of the check nodes from one side chain to increase the code rate.

In this chapter, we propose a new method to compensate for the rate loss of SC-LDPC codes with small chain lengths. Our interest in this problem is motivated by the theoretical question of whether or not the rate loss can be eliminated by small modifications to the boundary of the protograph? We tackle this question by attaching additional variable nodes to the check nodes at the chain boundary. Our goal is to increase the code rate while preserving the BP threshold of the original chain. The new variable nodes have degrees that are chosen from an irregular degree distribution. They connect to boundary check nodes using the edges that were previously removed by termination. Although we only consider a specific parameterized modification, our results suggest that a small modification to the boundary cannot make the rate loss negligible.

We also use linear programming (LP) to optimize the degree distribution so that the code rate is maximized while the BP threshold is preserved. Our results show that this can increase the code rate of the original SC-LDPC ensemble without degrading its BP threshold.

This chapter is organized as follows. In Section 2.2, we provide background about SC-LDPC codes. Section 2.3 presents the proposed code ensemble and Section 2.4 describes its DE equations. In Section 2.5, the ensemble is optimized. The results are presented in Section 2.6.

\subsection{Background}

A $(j, k)$-regular LDPC code is an LDPC code where all columns (resp. rows) of the parity-check matrix have $j$ (resp. $k$ ) ones. Equivalently, nodes (or vertices) 


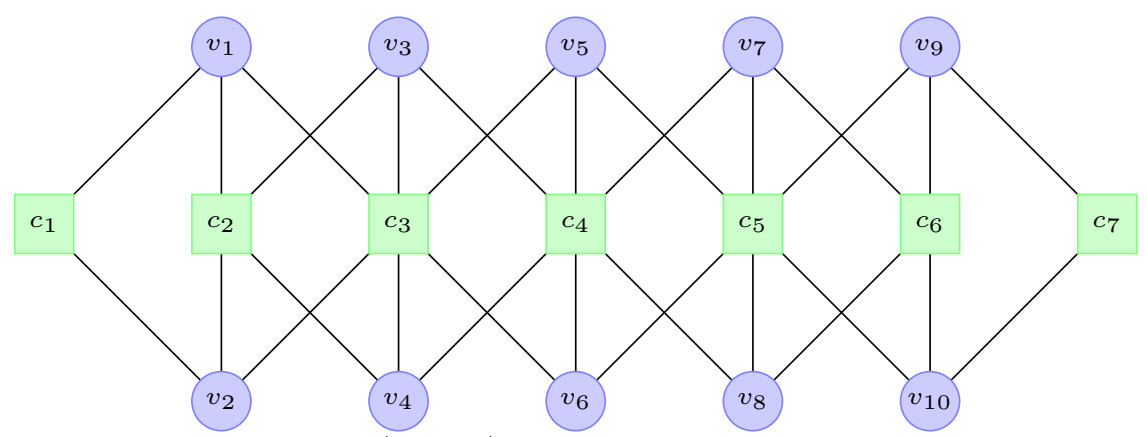

FigURE 2.1: $(3,6,5)$ spatially-coupled protograph.

in the Tanner graph associated with code bits (resp. parity checks) have degree $j$ (resp. degree $k)$. A $(j, k, L)$-regular SC-LDPC code is constructed by coupling $L$ copies of the $(j, k)$-regular LDPC ensemble. In this work, the coupling patterns are described using protographs. Figure 2.1 shows the protograph corresponding to a $(3,6,5) \mathrm{SC}$ code. Let $\left\{v_{j}\right\}_{1}^{N_{v}}$ and $\left\{c_{i}\right\}_{1}^{N_{c}}$ denote the variable nodes and check nodes in the protograph where $N_{c}$ and $N_{v}$ denote number of check nodes and number of variable nodes, respectively. For example, the $(3,6, L) \mathrm{SC}$ protograph has $2 L$ variable nodes and $L+2$ check nodes whereas $(5,10, L)$ SC protograph has the same number of variable nodes but $L+4$ check nodes. Protographs can be represented by their corresponding parity matrices. Let $H_{B}$ be the base parity-check matrix whose Tanner graph is the protograph. Then, $H_{B}$ for $(3,6, L)$ can be written as

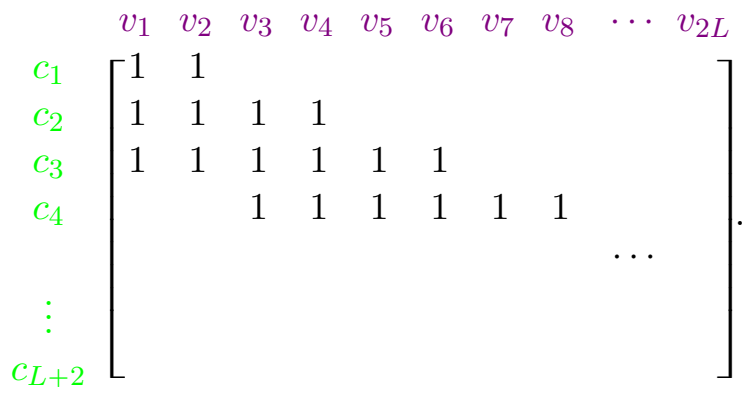

Given a protograph of a SC code, the code is constructed by lifting the protograph $M$ times as follows. First, every variable node $v_{j}$ and check node $c_{i}$ is copied $M$ times. In the lifted Tanner graph, let $v_{j}$ denote all the $M$ copies of $v_{j}$ in the Tanner graph 
and $c_{i}$ denote all the $M$ copies of $c_{i}$ in the Tanner graph. Then, variable nodes $v_{j}$ are connected to check nodes $c_{i}$ using a random permutation if and only if $v_{j}$ is connected to $c_{i}$ in the protograph. This is equivalent to replacing each 1 in the base parity-check matrix $H_{B}$ by a random $M \times M$ permutation matrix. The code rates of the $(3,6, L)$ and $(5,10, L) \mathrm{SC}$ codes are given by

$$
\begin{aligned}
& r_{(3,6, L)}=1-\frac{M N_{c}}{M N_{v}}=1-\frac{M(L+2)}{2 M L}=\frac{1}{2}-\frac{1}{L}, \\
& r_{(5,10, L)}=1-\frac{M N_{c}}{M N_{v}}=1-\frac{M(L+4)}{2 M L}=\frac{1}{2}-\frac{2}{L} .
\end{aligned}
$$

Although both $r_{(3,6, L)}$ and $r_{(5,10, L)}$ asymptotically converge to $1 / 2$ as $L \rightarrow \infty$, the convergence is slow enough to be a problem in practice.

\subsection{Code Design}

In this section, we propose a method to reduce the rate loss of $(3,6, L)$ and $(5,10, L)$ ensembles based on attaching additional variable nodes to Tanner graphs of ensembles. The objective is to maximize the code rate subject to the constraint that the BP threshold of the ensemble is not reduced. These additional variable nodes are called irregular variable nodes and their degree distribution, from the edge prospective, is denoted by $\lambda(x)$. The variable nodes associated with $\lambda(x)$ are connected to the boundary check nodes of $(j, k, L)$ so that the check degrees are increased to $k$.

Computing $\lambda(x)$ is formulated as an optimization problem to maximize the code rate such that the $\mathrm{BP}$ threshold of this new ensemble must be as large as the original $(j, k, L)$ ensemble. Using the symmetry of the protograph, we can avoid rate loss at one end of the chain by folding the protograph in the middle of its chains. For the $(3,6, L)$ example in Figure 2.1, the length of folded protograph equals $\lfloor(L+1) / 2\rfloor$. Due to symmetry, the DE analysis of original and folded protographs are equivalent.

Figure 2.2 shows the protograph structure of a $(3,6,12, \lambda)$ SC-LDPC code as 


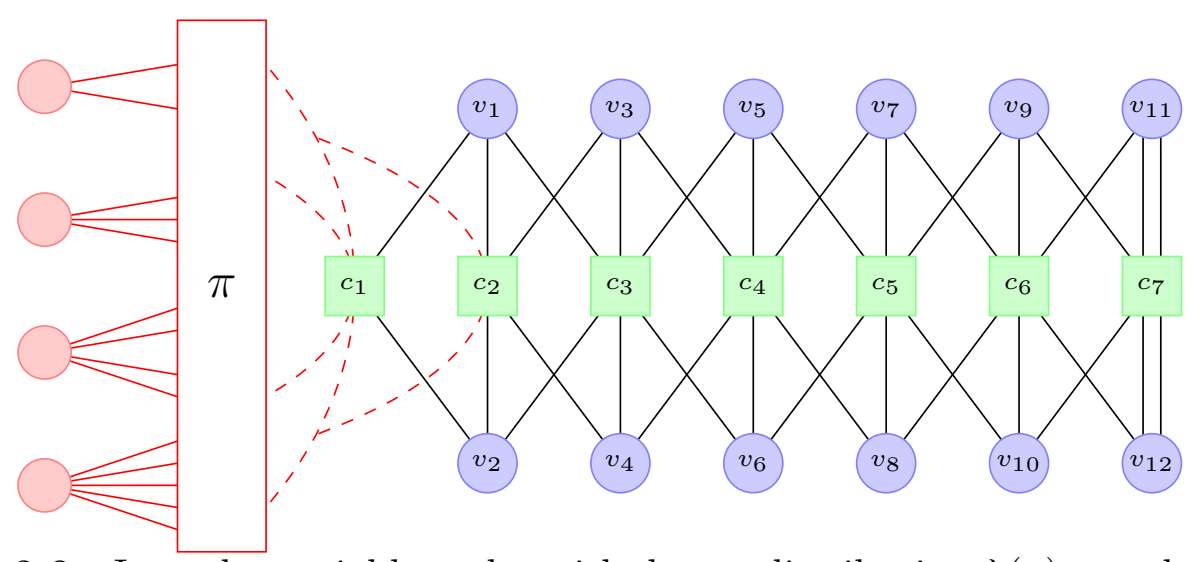

FiguRE 2.2: Irregular variable nodes with degree distribution $\lambda(x)$ attached to the folded $(3,6,12)$ SC chain.

defined below.

Definition 1. The $(j, k, L, \lambda) S C$-LDPC ensemble is defined as follows. Given a $(j, k, L) S C$-LDPC code, every boundary check node with degree $d<k$ is connected via $k-d$ edges to the group of irregular variable nodes whose degree distribution is given by $\lambda$.

\subsection{Density Evolution Equations}

In this section, we derive the density evolution equations for the $(3,6, L, \lambda)$ ensemble. Following similar steps, one can derive the density evolution equations for the $(5,10, L, \lambda)$ ensemble. Consider the BER performance of an LDPC code with BP decoding on the BEC channel. This quantity can be estimated by using density evolution to track the fraction of erasure messages sent from variable nodes to check nodes. The result is a recursive formula for the asymptotic erasure probability at every iteration of decoding process. One can think of the density evolution equations as defining a dynamical system whose state variables are the erasure probabilities of messages sent from variable nodes to check nodes.

The parity matrix $H_{B}$ corresponding to the folded $(3,6, L)$ protograph can be written as 


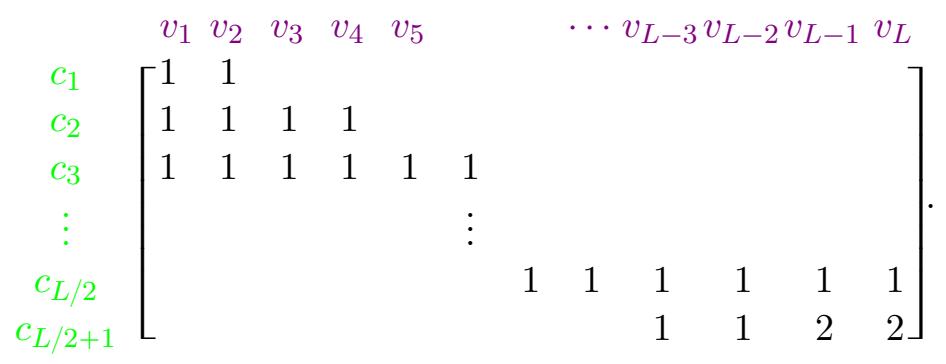

For every non-zero entry $H_{i, j}$ of $H_{B}$, there is one state variable that represents the average erasure probability of messages sent from variable nodes $v_{j}$ to check nodes $c_{i}$. This state variable is denoted by $x_{i, j}$.

The average erasure probability of messages sent from irregular variable nodes to check nodes $c_{1}$ and $c_{2}$ is denoted by a single state variable $z$. Let $z^{(\ell)}$ denote the value of state variable $z$ at the $\ell$-th iteration. Therefore, we can write

$$
z^{(\ell)}=\epsilon \sum_{i=1}^{L_{\max }} \lambda_{i}\left(\delta^{(\ell)}\right)^{i-1}
$$

where $\delta^{(\ell)}$ is the average erasure probability of messages sent from check nodes $c_{1}$ and $c_{2}$ to irregular variable nodes. Since the number of edges connecting check nodes $c_{1}$ to irregular variable nodes is twice the number of edges connecting check nodes $c_{2}$ to irregular variable nodes, we can write

$$
\delta^{(\ell)}=\frac{2}{3} y_{c_{1}}^{(\ell)}+\frac{1}{3} y_{c_{2}}^{(\ell)}
$$

where $y_{c_{1}}^{(\ell)}$ and $y_{c_{2}}^{(\ell)}$ denote the average erasure probabilities of messages sent from the check nodes $c_{1}$ and $c_{2}$, respectively, to irregular variable nodes. Therefore, we have

$$
\begin{aligned}
& y_{c_{1}}^{(\ell)}=\prod_{i=1}^{2}\left[1-\left(1-x_{1, i}^{(\ell-1)}\right)\right]\left(1-z^{(\ell-1)}\right)^{3}, \\
& y_{c_{2}}^{(\ell)}=\prod_{i=1}^{4}\left[1-\left(1-x_{2, i}^{(\ell-1)}\right)\right]\left(1-z^{(\ell-1)}\right) .
\end{aligned}
$$


Let $M_{j}$ denote the set of neighboring check nodes of the variable node $v_{j}$ in the protograph and $N_{i}$ denote the set of neighboring variable nodes of the check node $c_{i}$ in the protograph. Also, given $c_{i}$ is a neighbor of $v_{j}$, let $M_{j, i}$ be equal to $M_{j}-\left\{c_{i}\right\}$. And, given $v_{j}$ is a neighbor of $c_{i}$, let $N_{i, j}$ be equal to $N_{i}-\left\{v_{j}\right\}$. Let $x_{i, j}^{(\ell)}$ denote the value of state variable $x_{i, j}$ at $\ell^{\text {th }}$ iteration. Then, the density evolution equations for $x_{i, j}$ can be divided into the following cases:

Case I $\left(c_{1} \notin M_{i, j}\right.$ and $\left.c_{2} \notin M_{i, j}\right)$ :

$$
x_{i, j}^{(\ell)}=\epsilon \prod_{c_{i^{\prime}} \in M_{j}}\left(1-\prod_{v_{j^{\prime}} \in N_{i^{\prime}}}\left(1-x_{i^{\prime}, j^{\prime}}^{(\ell-1)}\right)^{H_{i^{\prime}, j^{\prime}}^{B}-\delta_{j, j^{\prime}}}\right)^{H_{i^{\prime}, j}^{B}-\delta_{i, i^{\prime}}}
$$

where $\delta$., denotes the Kronecker delta function,

Case II $\left(c_{1} \in M_{i, j}\right.$ and $\left.c_{2} \notin M_{i, j}\right)$ :

$$
\begin{gathered}
x_{i, j}^{(\ell)}=\epsilon\left[1-\left(1-z^{(\ell-1)}\right)^{4} \prod_{v_{j^{\prime}} \in N_{1, j}}\left(1-x_{i^{\prime}, j^{\prime}}^{(\ell-1)}\right)\right] \times \\
\prod_{c_{i^{\prime}} \in M_{j, i}, c_{i^{\prime}} \neq c_{1}}\left[1-\prod_{v_{j^{\prime}} \in N_{i^{\prime}, j}}\left(1-x_{i^{\prime}, j^{\prime}}^{(\ell-1)}\right)\right],
\end{gathered}
$$

Case III $\left(c_{1} \notin M_{i, j}\right.$ and $\left.c_{2} \in M_{i, j}\right)$ :

$$
\begin{gathered}
x_{i, j}^{(\ell)}=\epsilon\left[1-\left(1-z^{(\ell-1)}\right)^{2} \prod_{v_{j^{\prime}} \in N_{2, j}}\left(1-x_{i^{\prime}, j^{\prime}}^{(\ell-1)}\right)\right] \times \\
\prod_{c_{i^{\prime}} \in M_{j, i}, c_{i^{\prime}} \neq c_{2}}\left[1-\prod_{v_{j^{\prime}} \in N_{i^{\prime}, j}}\left(1-x_{i^{\prime}, j^{\prime}}^{(\ell-1)}\right)\right],
\end{gathered}
$$

Case IV $\left(c_{1} \in M_{i, j}\right.$ and $\left.c_{2} \in M_{i, j}\right)$ : 


$$
\begin{gathered}
x_{i, j}^{(\ell)}=\epsilon\left[1-\left(1-z^{(\ell-1)}\right)^{4} \prod_{v_{j^{\prime}} \in N_{1, j}}\left(1-x_{i^{\prime}, j^{\prime}}^{(\ell-1)}\right)\right] \times \\
{\left[1-\left(1-z^{(\ell-1)}\right)^{2} \prod_{v_{j^{\prime}} \in N_{2, j}}\left(1-x_{i^{\prime}, j^{\prime}}^{(\ell-1)}\right)\right] .}
\end{gathered}
$$

\subsection{Degree Distribution Optimization}

In this section, we optimize the $(j, k, L, \lambda)$ SC-LDPC ensemble. The optimization is over the $\lambda$ distribution and the constraints are defined in terms of the BP threshold of the SC-LDPC ensemble.

Let $n_{v, s c}$ and $n_{c}$ denote the number of variable nodes and check nodes, respectively, in the original SC Tanner graph. Therefore, $n_{v, s c}=2 L M$ and $n_{c}=M N_{c}$ where $N_{c}$ is equal to $L+2$ for $(3,6, L)$ SC ensemble and $L+4$ for $(5,10, L) \mathrm{SC}$ ensemble. Let $n_{v, \text { ir }}$ denote the number of irregular variable nodes drawn from $\lambda$. The number $n_{v, \text { ir }}$ can be computed by dividing the number of edges connecting irregular variable nodes to the original Tanner graph by the average node degree of irregular variable nodes. Let $l_{\text {avg }}$ denote the average node degree of these nodes and $N_{e}$ denote the number of edges connecting each copy of protograph to irregular variable nodes. We have $N_{e}=6$ corresponding to $(3,6, L)$ and $N_{e}=20$ for $(5,10, L)$. Therefore, we can write $n_{v, i r}=M N_{e} / l_{a v g}$.

Let $r_{\lambda}\left(l_{\text {avg }}\right)$ denote the rate of the optimized ensemble, where $l_{\text {avg }}$ is the average node degree of irregular variable nodes. We can write

$$
\begin{aligned}
r_{\lambda}\left(l_{\text {avg }}\right) & =1-\frac{n_{c}}{n_{v, i r}+n_{v, s c}}=1-\frac{M N_{c}}{\frac{M N_{e}}{l_{\text {avg }}}+2 L M} \\
& =1-\frac{N_{c}}{\frac{N_{e}}{l_{\text {avg }}}+2 L},
\end{aligned}
$$

where $N_{c}=L+2$ for $(3,6, L)$ and $N_{c}=L+4$ for $(5,10, L)$. It is observed that $r_{\lambda}\left(l_{\text {avg }}\right)$ 


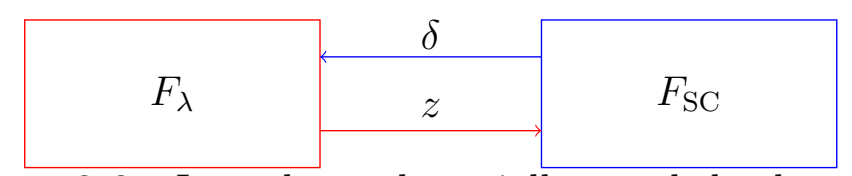

FiguRE 2.3: Irregular and spatially-coupled subsystems.

is a strictly decreasing function of $l_{\text {avg }}$. So, maximization of $r_{\lambda}\left(l_{\text {avg }}\right)$ is equivalent to minimization of $l_{\text {avg }}$. Moreover, one can show that

$$
l_{\text {avg }}=\frac{1}{\int_{0}^{1} \lambda(x) d x}=\frac{1}{\sum_{i=1}^{L_{\max }} \frac{1}{i} \lambda_{i}} .
$$

Therefore, maximization $r_{\lambda}\left(l_{\text {avg }}\right)$ reduces to the maximization of $\sum_{i=1}^{L_{\max }} \frac{1}{i} \lambda_{i}$ which is a linear objective function of decision variables $\left\{\lambda_{i}\right\}_{1}^{L_{\max }}$.

Let $\epsilon_{(j, k, L)}^{B P}$ denote the BP threshold of the $\mathrm{SC}(j, k, L)$ ensemble. Also, $\epsilon_{(j, k, L, \lambda)}^{B P}$ denotes the BP threshold of optimized $(j, k, L, \lambda)$ ensemble. The following optimization problem describes our objective and constraint to find the degree distribution $\lambda(x):$

$$
\sup _{\left\{\lambda_{i}\right\}_{1}^{L_{\text {max }}}} \sum_{i=1}^{L_{\max }} \frac{1}{i} \lambda_{i} \text { subject to } \epsilon_{(j, k, L, \lambda)}^{B P} \geqslant \epsilon_{(j, k, L)}^{B P}
$$

\subsubsection{Equivalent LP formulation}

In this section, we propose an equivalent linear programming (LP) for the optimization problem in (2.12).

The Tanner graph of $(j, k, L, \lambda)$ SC-LDPC codes can be divided into two interacting subsystems: (i) the subsystem consisting of irregular variable nodes drawn from $\lambda(x)$, denoted by $F_{\lambda}$ and (ii) the subsystem consisting of variable nodes and check nodes in the original Tanner graph of SC-LDPC ensemble, denoted by $F_{\mathrm{sc}}$. These two subsystems are depicted in Figure 2.3. The BP threshold of $F_{\text {sc }}$ is equal to $\epsilon_{(j, k, L)}^{B P}$ because it is identical to $(j, k, L)$ SC-LDPC. Therefore, the constraint in 


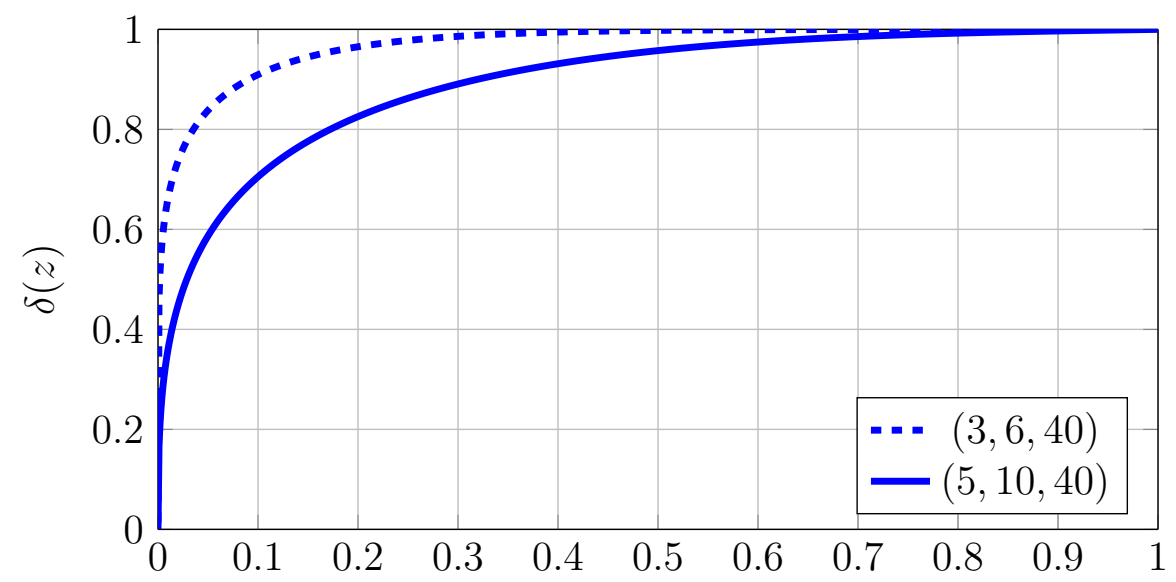

FiguRe 2.4: $\delta(z)$ versus $z$ fỡ the $(3,6,40)$ and $(5,10,40)$ ensembles.

(2.12) is reduced to the following constraint: The BP threshold of $F_{\lambda}$ having interacting dynamics with $F_{\mathrm{sc}}$ must be at least as large as $\epsilon_{(j, k, L)}^{B P}$.

Using the density evolution equations, the interacting dynamics between $F_{\lambda}$ and $F_{\mathrm{sc}}$ is determined using state variables exchanged between them. As shown in Figure 2.3 , during each iteration, $F_{\lambda}$ passes the value of the state variable $z$ to $F_{\mathrm{sc}}$ and $F_{\mathrm{sc}}$ passes $\delta$, the average erasure probability of messages sent from check nodes $c_{1}$ and $c_{2}$ to irregular variable nodes, to $F_{\lambda}$. In particular, to fully characterize $F_{\mathrm{sc}}$ from the point view of $F_{\lambda}$, it is sufficient to compute $\delta$ as a function of $z$.

Let $\delta\left(z_{0}\right)$ denote the $\delta$ value corresponding to $z=z_{0}$. To find $\delta\left(z_{0}\right)$, we use the density evolution equations as follows. We initialize all $x_{i, j}$ 's to be equal to $\epsilon_{(j, k, L)}^{B P}$ and $z$ to be equal to $z_{0}$. Then, we update $x_{i, j}$ 's using the equations in Section 2.4 except $z$ which is fixed at $z_{0}$. After convergence of $x_{i, j}$ 's to their fixed points, we report their corresponding $\delta$ value as $\delta\left(z_{0}\right)$. Figure 2.4 plots $\delta(z)$ versus $z$ for SC $(3,6,40)$ and $(5,10,40)$ ensembles. Given $z^{(l)}=\epsilon \sum_{i=1}^{L_{\max }} \lambda_{i}\left(\delta^{(l)}\right)^{i-1}$, a sufficient condition for $F_{\lambda}$ to have a $\mathrm{BP}$ threshold at least as large as $\epsilon_{(j, k, L)}^{B P}$ can be written as

$$
\epsilon_{(j, k, L)}^{B P} \sum_{i=1}^{L_{\max }} \lambda_{i} \delta(z)^{i-1}<z \quad \forall z \in\left(0, \epsilon_{(j, k, L)}^{B P}\right]
$$


Therefore, using (2.13), the optimization problem in (2.12) is reduced to

$$
\begin{gathered}
\sup _{\left\{\lambda_{i}\right\}_{1}^{L_{\max }}} \sum_{i=1}^{L_{\max }} \frac{1}{i} \lambda_{i} \\
\text { subject to } \epsilon_{(j, k, L)}^{B P} \sum_{i=1}^{L_{\max }} \lambda_{i} \delta(z)^{i-1}<z \forall z \in\left(0, \epsilon_{(j, k, L)}^{B P}\right] .
\end{gathered}
$$

In the following theorem, we derive an upper bound as a function of $\delta(z)$ for the optimized ensembles.

Theorem 1. For a given $\delta(z)$, an upper bound for the rate of the proposed code ensemble can be written

$$
r \leqslant 1-\frac{N_{c}}{\frac{N_{e}}{\epsilon}\left(1-\int_{0}^{1} \delta(z) d z\right)+2 L} .
$$

Proof. Using Area Theorem ((Richardson and Urbanke, 2008, Theorem 3.81)), to satisfy the constraint in (2.13), we have

$$
\int_{0}^{1} \delta(z) d z+\epsilon^{B P} \int_{0}^{1} \lambda(x) d x \leqslant 1 .
$$

Replacing $\int_{0}^{1} \lambda(x) d x=1 / l_{\text {avg }}$, we can write

$$
l_{\text {avg }} \geqslant \frac{\epsilon^{B P}}{1-\int_{0}^{1} \delta(z) d z} .
$$

Since $r\left(l_{\text {avg }}\right)=1-N_{c} /\left(\frac{N_{e}}{l_{\text {avg }}}+2 L\right)$, we conclude that

$$
r \leqslant 1-\frac{N_{c}}{\frac{N_{e}}{\epsilon^{B P}}\left(1-\int_{0}^{1} \delta(z) d z\right)+2 L} .
$$


Although the optimization problem in (2.14) is a LP problem, it includes infinite number of linear inequalities. In order to make it computationally tractable, we quantize the interval $\left(0, \epsilon_{(j, k, L)}^{B P}\right]$ uniformly. Let $Q$ denote the number of quantization points. An approximation of the LP problem in (2.14) using quantization is given by

$$
\begin{gathered}
\sup _{\left\{\lambda_{i}\right\}_{1}^{L_{\text {max }}}} \sum_{i=1}^{L_{\max }} \frac{1}{i} \lambda_{i} \\
\text { subject to } \epsilon_{(j, k, L)}^{B P} \sum_{i=1}^{L_{\max }} \lambda_{i} \delta\left(z_{j}\right)^{i-1}<z_{j}, \\
\forall z_{j}=\left(\frac{j}{Q}\right) \epsilon_{(j, k, L)}^{B P}, j \in\{1, \ldots, Q\} .
\end{gathered}
$$

\subsection{Results and Discussion}

In this section, we present the optimal $\lambda$ distribution for $(3,6,40)$ and $(5,10,40)$ ensembles found by solving the optimization problem in (2.16). Also, we report the achievable rates by these optimal degree distributions and compare them by the achievable rates using constant node degree distributions for each ensemble.

By density evolution, one can show that $\epsilon_{(3,6,40)}^{B P}$ is approximately equal to 0.48811 . Also, we have

$$
r_{(3,6,40)}=1-\frac{L+2}{2 L}=1-\frac{42}{80}=0.475 .
$$

The upper bound in $(2.15)$ for $(3,6,40)$ is equal to 0.483161 that is 0.008 larger than $r_{(3,6,40)}$. Let $r_{(3,6,40)}^{U}$ denote this upper bound. Solving the LP problem in (2.16) for $(3,6,40)$ with $Q=1000$ and $L_{\max }=40$, we have

$$
\lambda^{o p t}(x)=0.0193 x^{2}+0.3439 x^{3}+0.5310 x^{6}+0.1058 x^{7},
$$


Table 2.1: Code rates and the BP thresholds corresponding to constant node degree distributions for $(3,6,40)$ protograph.

\begin{tabular}{|c|c|c|c|}
\hline$\lambda(x)$ & $l_{\text {avg }}$ & $r_{\lambda}$ & $\epsilon_{\lambda}^{B P} \approx$ \\
\hline$x^{3}$ & 4 & 0.4847 & 0.45037 \\
\hline$x^{4}$ & 5 & 0.4827 & 0.47380 \\
\hline$x^{5}$ & 6 & 0.4815 & 0.48811 \\
\hline$x^{6}$ & 7 & 0.4806 & 0.48811 \\
\hline$x^{7}$ & 8 & 0.4799 & 0.48811 \\
\hline$\lambda^{\text {opt }}(x)$ & 5.5117 & 0.4820 & 0.48811 \\
\hline
\end{tabular}

and $r_{\left(3,6,40, \lambda^{o p t}\right)}=0.4820$. Therefore, we have

$$
\frac{r_{\left(3,6,40, \lambda^{o p t}\right)}-r_{(3,6,40)}}{r_{(3,6,40)}^{U}-r_{(3,6,40)}}=\frac{0.4820-0.4750}{0.4832-0.4750}=0.864 .
$$

This shows that using $\lambda^{\text {opt }}(x)$ in (2.18), we achieve $86 \%$ of the upper bound.

Here, we compare the performance of $\lambda^{\text {opt }}(x)$ in (2.18) with regular degree distributions in terms of their increase in code rate over the $(3,6,40)$ ensemble. Table 2.1 shows the code rates and the BP thresholds of the SC-LDPC ensembles $\left(3,6,40, x^{k}\right)$ for $k \in\{4, \ldots, 8\}$. The highest increase in code rate while not degrading the BP threshold happens for $\lambda(x)=x^{5}$. Of course, $\lambda^{o p t}(x)$ in (2.18) outperforms the best regular degree distribution $\lambda(x)=x^{5}$ but not by a significant margin.

For $(5,10,40)$ SC ensemble, $\epsilon_{(5,10,40)}^{B P}$ is approximately equal to 0.49943 by density evolution and $r_{(3,6,40)}=1-\frac{L+4}{2 L}=1-\frac{44}{80}=0.45$. The upper bound in (2.15) for $(5,10,40)$ is equal to 0.4579 and is denoted by $r_{(5,10,40)}^{U}$. Solving the LP problem in (2.16) with $Q=1000$ and $L_{\max }=150$, we have

$$
\begin{gathered}
\lambda^{\text {opt }}(x)=0.1240 x^{6}+0.1654 x^{7}+0.2202 x^{19}+0.0490 x^{20} \\
+0.0398 x^{52}+0.1757 x^{53}+0.1865 x^{146}+0.0391 x^{147}
\end{gathered}
$$

and $r_{\left(5,10,40, \lambda^{o p t}\right)}=0.4578$. Also, we have

$$
\frac{r_{\left(5,10,40, \lambda^{o p t}\right)}-r_{(5,10,40)}}{r_{(5,10,40)}^{U}-r_{(5,10,40)}}=\frac{0.4578-0.4500}{0.4579-0.4500}=0.9873
$$


Table 2.2: Code rates and the BP thresholds corresponding to constant node degree distributions for $(5,10,40)$ protograph.

\begin{tabular}{|c|c|c|c|}
\hline$\lambda(x)$ & $l_{\text {avg }}$ & $r_{\lambda}$ & $\epsilon_{\lambda}^{B P} \approx$ \\
\hline$x^{39}$ & 40 & 0.4534 & 0.49552 \\
\hline$x^{40}$ & 41 & 0.4533 & 0.49730 \\
\hline$x^{41}$ & 42 & 0.4532 & 0.49904 \\
\hline$x^{42}$ & 43 & 0.4532 & 0.49943 \\
\hline$x^{43}$ & 44 & 0.4531 & 0.49943 \\
\hline$\lambda^{\text {opt }}(x)$ & 17.4611 & 0.4578 & 0.49943 \\
\hline
\end{tabular}

Table 2.2 shows the results of attaching irregular variable nodes from constant node degree distributions. It is observed that the $\lambda^{\text {opt }}(x)$ in $(2.20)$ outperforms the best constant node degree distribution $\lambda(x)=x^{42}$.

Our results show that one natural approach to this problem does not appear to eliminate the rate loss. To prove this result, it seems that one would either have to try all possible boundary modifications or identify the fundamental reason why loss-less initialization is difficult.

\subsection{Conclusion}

In this chapter, we consider the rate-loss problem of SC-LDPC codes by optimizing (via LP) the degree distribution of additional variable nodes attached to the Tanner graphs of SC-LDPC codes. We show that the optimal degree distributions found by the LP increase the code rates of the SC-LDPC codes while not degrading their BP thresholds.

Although, we only report the results of a specific pattern of attaching extra variable nodes, we also examined a variety of other possible patterns. None of them showed any significant improvement over the method described in Section 2.3 and the results were very similar to those in Section 2.6.

Our results suggest that there is no small modification to the boundary of the 
SC-LDPC ensemble that makes the rate loss negligible. An interesting open question is whether or not one can make this statement precise and analytically prove it. 


\section{The Dynamics of Polarized Beliefs in Networks Governed by Viral Diffusion and Media Influence}

In this chapter, we study the evolution of polarized beliefs governed by the intertwined dynamics of viral diffusion and media influence in influence networks. It addresses the question of how different forms of influence interact with each other.

\subsection{Introduction}

To design effective marketing strategies that promote brand awareness, the adoption of innovations, or the popularity of new products, it is crucial to take into account the influence networks of targeted populations. In influence networks, nodes represent individuals and links describe influence relationships between them. The topology of an influence network specifies the underlying structure of influence relationships between individuals. Marketing strategies can be divided into the two broad categories: viral and media marketing. Viral marketing exploits the structures of influence networks to activate existing influence to target potential adopters, and form global cascades of adopters in influence networks. In particular, it is designed 
based on word-of-mouth and encourages individuals to share product information with their social contacts. Therefore, the medium for viral marketing is the links of social networks. On the other hand, media marketing treats all individuals as atomized objects of global media influence without taking into account their social networks. Media marketing is a broadcast mechanism that acts externally on influence networks because all the individuals receive the media influence directly from the same source. TV and newspaper ads are examples of media marketing while personalized referrals and recommendations are examples of viral marketing. Studying the dynamics of viral diffusion and media influence in networks helps firms to design their marketing campaigns according to the characteristics of diffusion dynamics in the targeted populations.

Studying the diffusion of new ideas, beliefs, and technologies, collectively called innovation, started about 120 years ago (Dearing (2008)). Marketing campaigns can be seen as external change agents that interact with the dynamics of innovation in influence networks to maximize their primary output measures. These goal of marketing is to effect maximal change with minimal cost (Dearing (2008)). Diffusion of innovations, viral marketing, has been studied extensively (Watts and Dodds (2007); Bakshy et al. (2011); Kempe et al. (2003); Leskovec et al. (2007); Kwak et al. (2010); Cha et al. (2010); Watts and Peretti (2007); Aral and Walker (2012); Cheng et al. (2014); Leskovec et al. (2006); Gomez Rodriguez et al. (2010); Sun et al. (2009); Ribeiro (2014); De Bruyn and Lilien (2008); Gomez Rodriguez and Schölkopf (2012); Du et al. (2013)). Aral et al. studied effects of viral product design strategies on creating word-of-mouth dynamics (Aral and Walker (2011)). In particular, they designed and conducted a randomized field experiment testing the effectiveness of active-personalized referrals and passive-broadcast notifications. They found that passive-broadcast viral messaging capabilities induce higher increase in social contagion compared to active-personalized messaging. Leskovec et al. studied dynamics 
of viral marketing in a recommendation network with 4 million people (Leskovec et al. (2007)). They found that most recommendation chains do not turn into large cascades, and viral marketing is more effective for expensive products recommended to small and well-connected communities. Also, they explained the propagation of recommendations and cascade sizes in this network by a stochastic model.

In the diffusion of innovation research community, one approach to diffusion maximization is focus first on determining influential individuals (or influentials). Influentials are opinion leaders who have the credibility to influence a disproportionately large number of individuals. Aral et al. showed that in a representative sample of 1.3 million Facebook users, influential individuals are less susceptible to influence compared to noninfluential individuals (Aral and Walker (2012)). Also, They observed that influentials cluster in the network whereas noninfluential individuals do not. Watts et al. studied the role of influentials in marketing and the formation of public opinion using a series of computer simulations of interpersonal influence processes (Watts and Dodds (2007)). In particular, they studied the conditions under which local cascades can turn into global cascades, and showed that under most conditions, the global cascades are driven by the critical mass of individuals who are easily influenced and not by influentials. Bakshy et al. investigated the word-ofmouth dynamics among Twitter users using the Twitter follower graph (Bakshy et al. (2011)). They found that users with a large number of followers and users who have been influential in the past are the ones that generate the largest cascades. Also, they concluded that marketers can reliably benefit from word-of-mouth diffusion if large numbers of potential influencers are targeted. The diffusion process is also studied extensively in the context of epidemiology (Sanatkar et al. (2016, 2015); Sahneh et al. (2013); Bogu et al. (2003); Pastor-Satorras and Vespignani (2001); Mieghem et al. (2009)). They analyzed propagation of diseases over networks based on the stages of diseases in hosts where the transitions between these stages are modeled by several 
dynamics.

A myriad of studies show that the decision process of individuals is affected by mixtures of interpersonal and media influence (Watts and Dodds (2007)). Analyzing empirical diffusion patterns over seven different online domains, Goel et al. concluded that these diffusion patterns motivate models that explicitly take into account media marketing in addition to viral marketing (Goel et al. (2012)). While most studies of the diffusion process assume person-to-person networks as the only medium for diffusion of innovations, a number of recent studies consider both global influence by external sources and interpersonal networks as mechanisms of diffusion (Goel et al. (2016); Myers et al. (2012); Farajtabar et al. (2014); Kleineberg and Boguñá (2014)). Goel et al. showed that popular events regularly grow via combination of both broadcast, media marketing, and viral diffusion (Goel et al. (2016)). Myers et al. presented a model of information emergence in networks in which information can reach an individual through his neighbors or via the influence of an external source (Myers et al. (2012)). They fitted the parameters of their model to a complete one month trace of the emergence of URL mentions in the Twitter network. They found that only about $71 \%$ of the tweets with URL mentions can be explained by viral propagation in the network of Twitter's followers, and the remaining $21 \%$ are due to external sources.

Also, Farajtabar et al. modeled intensities of endogenous and exogenous events in networks of individuals by multivariate Hawkes processes (Farajtabar et al. (2014)). They drove a time-dependent linear relationship that describes the relationship between the overall network activity and the intensity of exogenous events. Also, they computed the required level of external influence applied to the network to attain a desired activity level using a convex optimization framework. Kleineberg et al. presented a two-layer model for the evolution of online social networks under viral spreading mechanisms and mass media influence (Kleineberg and Boguná (2014)). 
Based on the empirical validation of their model, they found that viral influence is 4-5 times stronger than mass media influence for the studied online social network.

In this chapter, we propose a stochastic system to model adoption process of polarized beliefs governed by viral diffusion and media influence at the individual level. The primary difference between our model and other recent studies (Goel et al. (2012, 2016); Myers et al. (2012); Farajtabar et al. (2014); Kleineberg and Boguña (2014)) that model both interpersonal and media influence is the following: we consider a third state, the so called negative state, to represent those individuals who hold positions against the innovation in addition to two existing states (neutral (susceptible) and positive (adoption)) in (Goel et al. (2012, 2016); Myers et al. (2012); Farajtabar et al. (2014); Kleineberg and Boguña (2014)). To the best of our knowledge, our proposed stochastic system is the first model that takes into account both interpersonal and media influence for polarized beliefs in influence networks. In many real-world scenarios such as political debates, same-sex marriage, abortion and gun control, in additions to pro and neutral individuals, there exists a third group of individuals that are neither positive, adopter, nor neutral, susceptible, but against the innovation (Guerra et al. (2013); Yardi and Boyd (2010)). Our proposed model aims as describing such polarized belief dynamics over influence networks. For example, since the recent emergence of smart-watches in 2013, people have had polarized ideas toward them. While many people support using them because of the convenience that comes with receiving notifications on their wrists, many people think that we are already very preoccupied with our smart-phones in our daily lives, and we do not need another device.

First, we propose a stochastic system to model the dynamics of polarized beliefs at the individual level. This stochastic system is a Markov chain and is called the individual-based stochastic (IN-STOCH) system. This system is described by a set of individual-based transition events that govern dynamics of polarized belief prop- 
agation in influence networks. Then, using a mean-field analysis, we approximate the IN-STOCH system in the large population limit by deterministic differential equations so called the homogeneous mean-field (HOM-MEAN) and the heterogeneous mean-field (HET-MEAN) systems for exponential and scale-free networks, respectively. The HOM-MEAN system is based on homogeneity and randomness assumptions of influence networks. However, for the HET-MEAN system, we do not assume homogeneity for influence networks. We show that the HOM-MEAN system has at most three equilibrium points and one of these is always unstable. Then, we prove that the stability of the other two depends on the parameters of the model and the average node degrees in the influence network. In particular, one of the two stable equilibria has a vanishing fraction of negative individuals. This is the desired equilibrium point based on our chosen output measure. Based on the stability of this equilibrium point, we derive conditions for local and global convergence, of the fraction of negative individuals, to zero. Critical values of model parameters corresponding to these conditions characterize different phase transitions of polarized dynamics.

We show that the HET-MEAN system has two equilibria. Then, we derive the necessary and sufficient conditions for local exponential stability of the equilibrium point whose asymptotic fraction of negative individuals is zero. Also, we apply our theoretical results for the HET-MEAN system to the Barabasi-Albert networks (Barabasi and Albert (1999)), and we simplify the stability conditions for this equilibrium point. Finally, we validate our predictions by simulating the IN-STOCH system over random regular, Watts-Strogatz and Barabasi-Albert networks as well as the ego-Facebook network (Leskovec and Mcauley (2012)).

The rest of this chapter is organized as follows. In Section 3.2, we propose the IN-STOCH system to model the individual-based dynamics of polarized beliefs. Section 3.3 contains the HOM-MEAN system to approximate the IN-STOCH system for 


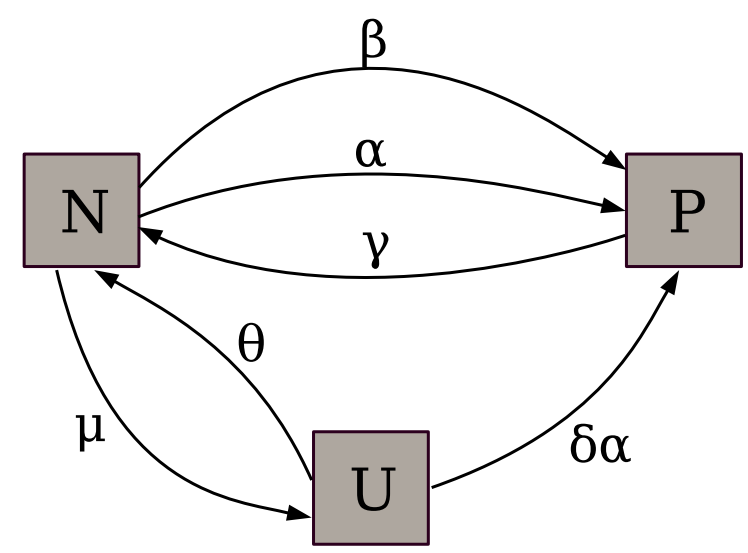

Figure 3.1: Flowchart of the individual-based stochastic system. A neutral node may become positive with a probability $\beta$ per its positive neighbors and because of media influence with a probability $\alpha$. A positive node may return to the neutral state with a probability $\gamma$. A neutral node may become negative with a probability $\mu$ per its negative neighbors. A negative node may return to the neutral state with a probability $\theta$ and to the positive state with a probability $\delta \alpha$.

exponential networks at population level. In Section 3.4, we derive the HET-MEAN system to approximate the IN-STOCH system for scale-free networks. Finally, in Section 3.5, we use simulation results to validate our theoretical analysis.

\subsection{Individual-Based Stochastic System}

In this section, we propose a stochastic model, called the individual-based stochastic (IN-STOCH) system, of the adoption process of polarized dynamics at the individual level. This stochastic system assumes that individuals are influenced via the links of influence networks or through the global mass media. In social networks such as Facebook where individual are either associated (e.g., friends) or unassociated, the influence network is assumed to be an undirected graph. In social networks such as Twitter where individuals follow one another, the influence network is assumed to be a directed graph. In this work, we consider only undirected influence networks. The extension to directed influence networks is straightforward. 
In this model, at any given time, each node is in one of the three states: neutral $(\mathrm{N})$, positive $(\mathrm{P})$ and negative $(\mathrm{U})$. Each state represents a different mindset of individuals in the adoption process. An individual in the neutral state, does not have a strong opinion towards the innovation, or has not received any information about the innovation. On the other hand, positive individuals are the ones who have adopted the innovation. For example, they already bought the product and are satisfied with it, or are willing to buy it. The negative state represents those individuals with negative opinions towards the innovation or product. For instance, they are who already bought the product but are not happy with it, or have been convinced by some of their friends not to buy it.

Polarized beliefs propagate among individuals based on the following mechanisms (as shown in Figure 3.1). A neutral individual may become positive due to viral adoption or media influence. Viral (or word-of-mouth) adoption, is modeled by a stochastic reaction process. A neutral node may virally adopt the innovation with a probability $\beta$ per each of its positive neighbors. On the other hand, media influence is modeled by a stochastic diffusion process. Under this process, a neutral individual will become positive with probability $\alpha$ because of media influence. It is noted that the media influence probability $\alpha$ is identical for all individuals and independent of the states of their neighbors.

A neutral individual may become negative under a word-of-mouth process with a probability $\mu$ per each of its negative neighbors. A negative individual may become neutral with a probability $\theta$. One cause for this transition can be a series of compensating actions taken by marketing campaigns to make negative individuals neutral. Here, we assume that mass-media for a negative user is not as effective as mass-media for a neutral user. A negative user may become positive with a probability $\delta \alpha$ under media influence, where $\delta \in[0,1]$. A positive individual may become neutral with a probability $\gamma$. One example for this stochastic process can be transitions of 
those individuals who have bought the product, but after using if for a while, are not satisfied with it.

The IN-STOCH system is a homogeneous Markov chain on $\{P, N, U\}^{C}$, where $C$ denotes the number of individuals; it governs the state transitions of individuals with respect to the states of their neighbors. For any given node $i$, let $R_{i}$ denote the set of its neighbors. Also, $P_{i, 0}(t), P_{i,+}(t)$ and $P_{i,-}(t)$ denote the probability of node $i$ being at state neutral, positive and negative, respectively, at time step $t$. The state of node $j$ at time step $t$ is denoted by $x_{j}(t)$. Let $R_{i,+}(t):=\sum_{j \in R_{i}} \mathbb{1}\left(x_{j}(t)=P\right)$ and $R_{i,-}(t):=\sum_{j \in R_{i}} \mathbb{1}\left(x_{j}(t)=U\right)$, where $\mathbb{1}$ denotes the indicator function. Therefore, the IN-STOCH system is equivalent to the following Markov chain:

$$
\begin{aligned}
P_{i, 0}(t+1) & =\gamma P_{i,+}(t)+\theta P_{i,-}(t) \\
& +\left(1-\alpha-\beta R_{i,+}(t)-\mu R_{i,-}(t)\right) P_{i, 0}(t), \\
P_{i,+}(t+1) & =\left(\alpha+\beta R_{i,+}(t)\right) P_{i, 0}(t)+\delta \alpha P_{i,-}(t) \\
& +(1-\gamma) P_{i,+}(t), \\
P_{i,-}(t+1) & =\mu R_{i,-}(t) P_{i, 0}(t)+(1-\theta-\delta \alpha) P_{i,-}(t) .
\end{aligned}
$$

For simplicity, this model considers only three belief states and a restricted set of transitions. These transitions were chosen based on the adoption scenarios we envision.

The goal of intervening agencies and marketing campaigns is to steer a targeted population to a targeted state. These agencies intervene to alter the dynamics of influence networks and maximize their primary output measures. Many different output measures can be chosen by intervening agencies based on the characteristics of targeted populations and innovations. Some examples of these output measures are driving the overall percentage of adoption to a certain level or making the adoption level homogeneous across users belonging to different communities. In this chapter, the asymptotic fraction of individuals with negative state is chosen as the primary 
output measure. In other words, we design the intervention process realized by a combination of viral diffusion and media influence to minimize (e.g., set to zero) the asymptotic fraction of negative individuals. Our main reason for choosing this specific output measure is that the lack of negative individuals prevents the viral dynamics from drifting towards the negative state.

\subsection{Exponential Networks}

In this section, we use a mean-field approach to derive a set of deterministic differential equations so called the homogeneous mean-field (HOM-MEAN) system to approximate the IN-STOCH system at population level for exponential networks. Networks with exponential degree distributions are called exponential networks. An exponential degree distribution peaks at the average node degree $\langle k\rangle$, and decays exponentially fast for node degrees $k$ when $k \gg\langle k\rangle$ or $k \ll\langle k\rangle$. The aim is to predict macroscopic measures of the IN-STOCH system. Then, we study asymptotic behaviors of targeted populations by examining the stability of the equilibrium points of the HOM-MEAN system. We show that this dynamical system has at most 3 equilibrium points. Based on our chosen output measure for marketing campaigns, the desirable equilibrium is the one whose fraction of negative individuals is zero. We derive critical values for dynamical phase transitions of populations by studying local and global stability of this equilibrium point. In particular, two sets of conditions are derived that guarantee the local and global convergence of the fraction of negative individuals to zero.

Let $n(t), p(t)$, and $u(t)$ be the fraction of neutral, positive and negative individuals 
at time $t$. Therefore, we can write

$$
\begin{aligned}
& n(t):=\frac{1}{C} \sum_{i=1}^{C} \mathbb{1}\left(x_{i}(t)=N\right), \\
& p(t):=\frac{1}{C} \sum_{i=1}^{C} \mathbb{1}\left(x_{i}(t)=P\right), \\
& u(t):=\frac{1}{C} \sum_{i=1}^{C} \mathbb{1}\left(x_{i}(t)=U\right),
\end{aligned}
$$

and $n(t)+p(t)+u(t)=1$. For a variable that depends on time, such as $n(t)$, we use $n$ to denote $n(t)$ and $\dot{n}$ to denote the time-derivative $n^{\prime}(t)$. Thus, the HOM-MEAN dynamical system is described with the following coupled differential equations in continuous-time:

$$
\begin{aligned}
& \dot{p}=k \beta p n+\alpha n-\gamma p+\delta \alpha u \\
& \dot{n}=-k \beta p n-\alpha n+\gamma p-k \mu n u+\theta u \\
& \dot{u}=k \mu n u-\theta u-\delta \alpha u .
\end{aligned}
$$

The HOM-MEAN system is derived assuming the independence between states of individuals. Also, it assumes that the fluctuations in exponential degree distributions around average node degrees can be neglected. This assumption is consistent with the empirical literature on influentials (Brock and Durlauf (2001)). That is node degree distributions of influence networks display a relatively little variation around their averages. For influence networks with non-local connectivity, we expect that the derived analytical results at population level based on the HOM-MEAN system, will approximate the behavior of targeted population (Pastor-Satorras and Vespignani (2001)). Using $n+p+u=1$ reduces (3.3) to the 2 -dimensional system:

$$
\begin{aligned}
\dot{n} & =k \beta n^{2}-(k \beta+\alpha+\gamma) n+(k \beta-k \mu) n u \\
& +(\theta-\gamma) u+\gamma, \\
\dot{u} & =k \mu n u-(\theta+\delta \alpha) u .
\end{aligned}
$$




\subsubsection{Equilibrium Points}

To study the steady state behavior of the HOM-MEAN system, we start by finding its equilibrium points. We want to choose a set of values for parameters that ensures the stability of the equilibrium points with no negative individuals. The equilibrium points of the dynamical system are found by imposing the stationary conditions. That is $\dot{n}=0$ and $\dot{u}=0$. Let $(\bar{n}, \bar{u})$ be an equilibrium point of this dynamical system. From

$$
\dot{u}=k \mu \bar{n} \bar{u}-(\theta+\delta \alpha) \bar{u}=0
$$

we see that either $\bar{u}=0$ or $\bar{n}=(\theta+\delta \alpha) /(k \mu)$. Replacing $\bar{u}=0$ in

$$
\begin{aligned}
& \dot{n}=k \beta \bar{n}^{2}-(k \beta+\alpha+\gamma) \bar{n} \\
& +(k \beta-k \mu) \bar{n} \bar{u}+(\theta-\gamma) \bar{u}+\gamma=0,
\end{aligned}
$$

we have

$$
k \beta \bar{n}^{2}-(k \beta+\alpha+\gamma) \bar{\gamma}+\gamma=0
$$

Lemma 1. The dynamical system in Equation (3.4) has at least one equilibrium point with no negative individuals if and only if

$$
\alpha+\gamma \leqslant k \beta+\sqrt{(k \beta+\alpha+\gamma)^{2}-4 k \beta \gamma}
$$

Proof. The candidates for the equilibrium points with no negative individuals are the solutions of (3.7). Equation (3.7) has a real solution if and only if $(k \beta+\alpha+$ $\gamma)^{2}-4 k \beta \gamma \geqslant 0$. Let $\Delta:=(k \beta+\alpha+\gamma)^{2}-4 k \beta \gamma$. Now, we show that $\Delta$ is always non-negative. We have

$$
(k \beta-\gamma)^{2}+\alpha^{2}+\gamma^{2}+2 k \beta \alpha+2 \alpha \gamma \geqslant 0 .
$$

By expanding and rearranging, we can show that

$$
\Delta=k^{2} \beta^{2}+\alpha^{2}+\gamma^{2}-2 k \beta \gamma+2 k \beta \alpha+2 \alpha \gamma
$$


Therefore, (3.7) has at least one real solution, and at maximum two solutions that can be written as

$$
\begin{aligned}
& \bar{n}_{1}=\frac{k \beta+\alpha+\gamma+\sqrt{\Delta}}{2 k \beta}, \\
& \bar{n}_{2}=\frac{k \beta+\alpha+\gamma-\sqrt{\Delta}}{2 k \beta} .
\end{aligned}
$$

Both $\bar{n}_{1}$ and $\bar{n}_{2}$ are always non-negative because $\sqrt{\Delta} \leqslant k \beta+\alpha+\gamma \cdot\left(\bar{n}_{1}, 0\right)$ and $\left(\bar{n}_{2}, 0\right)$ are equilibrium points if $\bar{n}_{1}$ and $\bar{n}_{2}$, respectively, are less than or equal to one. It is because the state space of the HOM-MEAN system is $[0,1] \times[0,1]$. Now, we show that if (3.8) is satisfied, $\bar{n}_{2}$ will be less than or equal to 1 . Because $\bar{n}_{2} \leqslant \bar{n}_{1}$, if $\bar{n}_{2} \leqslant 1$, then the HOM-MEAN system has at least one equilibrium point with no negative individuals. $\bar{n}_{2} \leqslant 1$ if and only if

$$
\alpha+\gamma \leqslant k \beta+\sqrt{\Delta}
$$

The HOM-MEAN system may have another equilibrium point with non-zero fraction of negative individuals. Replacing $\bar{n}=(\theta+\delta \alpha) /(k \mu)$ in

$$
\begin{aligned}
& \dot{n}=k \beta \bar{n}^{2}-(k \beta+\alpha+\gamma) \bar{n} \\
& +(k \beta-k \mu) \bar{n} \bar{u}+(\theta-\gamma) \bar{u}+\gamma=0,
\end{aligned}
$$

we have

$$
\bar{u}=\frac{-k \mu \gamma+(\delta \alpha+\theta)(k \beta+\alpha+\gamma)-\frac{\beta}{\mu}(\theta+\delta \alpha)^{2}}{k \beta(\theta+\delta \alpha)-k \mu(\gamma+\delta \alpha)}
$$

\subsubsection{Stability of Equilibrium Points}

In this section, we study the stability of the equilibrium points of the HOM-MEAN system. In particular, we show that, of the two possible equilibrium points whose fraction of negative individuals is zero, only $\left(\bar{n}_{2}, 0\right)$ can be locally stable, and $\left(\bar{n}_{1}, 0\right)$ 
is always unstable. Also, conditions for global asymptotic stability of $\left(\bar{n}_{2}, 0\right)$ are derived.

An equilibrium point of a dynamical system is locally exponentially stable if and only if the real parts of all eigenvalues of its Jacobian matrix, computed at the equilibrium point, are negative, and it is unstable if at least one of the eigenvalue of the Jacobian matrix, computed at the equilibrium point, has a positive real part (Khalil (2000)). Let $A\left(n^{*}, u^{*}\right)$ be the Jacobian matrix of the HOM-MEAN system in (3.4) at point $\left(n^{*}, u^{*}\right)$, where $A_{11}\left(n^{*}, u^{*}\right)=\left.\frac{\partial n^{*}}{\partial n}\right|_{\left(n^{*}, u^{*}\right)}, A_{12}\left(n^{*}, u^{*}\right)=\left.\frac{\partial n^{*}}{\partial u}\right|_{\left(n^{*}, u^{*}\right)}$, $A_{21}\left(n^{*}, u^{*}\right)=\left.\frac{\partial u}{\partial n}\right|_{\left(n^{*}, u^{*}\right)}$, and $A_{22}\left(n^{*}, u^{*}\right)=\left.\frac{\partial u}{\partial u}\right|_{\left(n^{*}, u^{*}\right)}$. Hence, we have

$$
\begin{aligned}
& A_{11}\left(n^{*}, u^{*}\right)=2 k \beta n^{*}+(k \beta-k \mu) u^{*}-(k \beta+\alpha+\gamma), \\
& A_{12}\left(n^{*}, u^{*}\right)=(k \beta-k \mu) n^{*}+\theta-\gamma, \\
& A_{21}\left(n^{*}, u^{*}\right)=k \mu u^{*}, \\
& A_{22}\left(n^{*}, u^{*}\right)=k \mu n^{*}-\theta-\delta \alpha .
\end{aligned}
$$

Computing the matrix $A$ at $\left(\bar{n}_{1}, 0\right)$, we have

$$
\begin{aligned}
& A_{11}=\sqrt{\Delta} \\
& A_{12}=\frac{\beta-\mu}{2 \beta}(k \beta+\alpha+\gamma+\sqrt{\Delta})+\theta-\gamma, \\
& A_{21}=0 \\
& A_{22}=\frac{\mu}{2 \beta}(k \beta+\alpha+\gamma+\sqrt{\Delta})-\theta-\delta \alpha .
\end{aligned}
$$

The Jacobian matrix computed at $\left(\bar{n}_{1}, 0\right)$ is an upper triangular matrix. Also, it is a real matrix because $\Delta \geqslant 0$ as we showed in Lemma 1 . Therefore, its two eigenvalues are real and equal to $A_{11}$ and $A_{22}$. We can conclude that $\left(\bar{n}_{1}, 0\right)$ is always unstable 
because $A_{11}$ is always positive. The Jacobian matrix at $\left(\bar{n}_{2}, 0\right)$ can be computed as:

$$
\begin{aligned}
& A_{11}=-\sqrt{\Delta} \\
& A_{12}=\frac{\beta-\mu}{2 \beta}(k \beta+\alpha+\gamma-\sqrt{\Delta})+\theta-\gamma, \\
& A_{21}=0 \\
& A_{22}=\frac{\mu}{2 \beta}(k \beta+\alpha+\gamma-\sqrt{\Delta})-\theta-\delta \alpha .
\end{aligned}
$$

The two eigenvalues of the Jacobian matrix are real and equal to $A_{11}$ and $A_{22}$ because the Jacobian matrix is upper triangular and $\Delta$ is always non-negative. The local stability of $\left(\bar{n}_{2}, 0\right)$ is only determined by $A_{22}$ because $A_{11}$ is always negative. In the following theorem, we derive conditions under which $\left(\bar{n}_{2}, 0\right)$ is locally exponentially stable.

Theorem 2. If (3.8) in Lemma 1 is satisfied, then $\left(\bar{n}_{2}, 0\right)$ is locally exponentially stable if and only if

$$
\begin{aligned}
& \text { (a) } \mu<\frac{2 \beta(\theta+\delta \alpha)}{k \beta+\alpha+\gamma} \text {, or } \\
& \text { (b) } \mu>\frac{2 \beta(\theta+\delta \alpha)}{k \beta+\alpha+\gamma} \text { and } \\
& \gamma<\frac{1}{\mu-\frac{1}{k}(\delta \alpha+\theta)}\left((\delta \alpha+\theta)\left(\beta+\frac{1}{k} \alpha\right)-\frac{\beta}{k \mu}(\theta+\delta \alpha)^{2}\right) .
\end{aligned}
$$

Proof. If (3.8) in Lemma 1 is satisfied, then $\left(\bar{n}_{2}, 0\right)$ is locally exponentially stable if and only if $A_{22}$ is negative. That is

$$
\frac{\mu}{2 \beta}(k \beta+\alpha+\gamma-\sqrt{\Delta})-\theta-\delta \alpha<0 .
$$

The inequality in (3.19) holds if and only if

$$
-\sqrt{\Delta}<\frac{2 \beta}{\mu}(\theta+\delta \alpha)-(k \beta+\alpha+\gamma) .
$$


If the RHS of (3.20) is positive, the inequality in (3.20) holds because its LHS is always negative. The RHS of (3.20) is positive if and only if

$$
\mu<\frac{2 \beta(\theta+\delta \alpha)}{k \beta+\alpha+\gamma}
$$

which yields the constraint (a). However, if the RHS of (3.20) is negative, then the inequality in (3.20) is satisfied if and only if

$$
\Delta>\left(\frac{2 \beta}{\mu}(\theta+\delta \alpha)-(k \beta+\alpha+\gamma)\right)^{2} .
$$

The inequality in (3.22) is equivalent to

$$
k \mu \gamma<\delta \alpha(k \beta+\alpha+\gamma)+\theta(k \beta+\alpha+\gamma)-\frac{\beta}{\mu}(\theta+\delta \alpha)^{2}
$$

which yields the constraint (b).

The constraints in Theorem 2 show two different regimes that both guarantee convergence of the fraction of negative individuals to zero. In the constraint (a), the condition for this convergence is characterized by $\mu$. We call the regime corresponding to this constraint, regime 1 . On the other hand, in the constraint (b), $\gamma$ characterizes the convergence to zero for the fraction of negative individuals. The regime corresponding to this constraint is called regime 2. We define negative-free thresholds with respect to the conditions in Theorem 2 to represent the desired phase transitions that guarantee the convergence to zero for the fraction of negative individuals. Let $\eta_{1}:=\frac{2 \beta(\theta+\delta \alpha)}{k \beta+\alpha+\gamma}$ denote the negative-free threshold for regime 1 . Also, let $\eta_{2}:=\frac{1}{\mu-\frac{1}{k}(\delta \alpha+\theta)}\left((\delta \alpha+\theta)\left(\beta+\frac{1}{k} \alpha\right)-\frac{\beta}{k \mu}(\theta+\delta \alpha)^{2}\right)$ denote the negative-free threshold for regime 2 . In regime $1,\left(\bar{n}_{2}, 0\right)$ is exponentially stable if and only if $\mu<\eta_{1}$. In regime $2,\left(\bar{n}_{2}, 0\right)$ is exponentially stable if and only if $\mu>\eta_{1}$ and $\gamma<\eta_{2}$.

HOM-MEAN systems with larger negative-free thresholds are more resistant to 
processes that push individuals towards the negative state. Therefore, intervening agencies should design their intervention process to make negative-free thresholds as large as possible. In regime 1, increasing the average node degree decreases the negative-free threshold, $\eta_{1}$, so that decreasing the chance of stability of $\left(\bar{n}_{2}, 0\right)$. In other words, increasing the average node degree helps the word-of-mouth process that causes individuals become negative. In regime 2 , the condition (b) in Theorem 2 reduces to

$$
\gamma<\frac{\beta}{\mu}(\delta \alpha+\theta)
$$

as $k \rightarrow \infty$. Equation (3.24) shows the significance of both viral diffusion and media influence for highly connected influence networks.

Theorem 2 provides us with the necessary and sufficient conditions of the local stability of $\left(\bar{n}_{2}, 0\right)$, but not its global stability. In the following theorem, we derive the conditions that ensure the equilibrium point with a non-zero fraction of negative individuals,

$$
\begin{aligned}
& \bar{n}=(\theta+\delta \alpha) /(k \mu) \\
& \bar{u}=\frac{-k \mu \gamma+(\delta \alpha+\theta)(k \beta+\alpha+\gamma)-\frac{\beta}{\mu}(\theta+\delta \alpha)^{2}}{k \beta(\theta+\delta \alpha)-k \mu(\gamma+\delta \alpha)}
\end{aligned}
$$

does not lie in the state space of the HOM-MEAN system.

Theorem 3. If one of the conditions in Theorem 2 is satisfied, then the $\left(\bar{n}_{2}, 0\right)$ point is the only stable equilibrium point of the HOM-MEAN system if one of the following 
conditions is satisfied:

$$
\begin{aligned}
& \text { (a) } \mu<\frac{1}{k}(\theta+\delta \alpha), \\
& \text { (b) } \mu<\mu_{0} \text { and } \gamma>\gamma_{0}, \\
& \text { (c) } \mu>\mu_{0} \text { and } \gamma<\gamma_{0}, \\
& \text { (d) } \mu<\mu_{0}, \gamma<\gamma_{0} \text { and } \\
& (\delta \alpha+\theta)(\alpha+\gamma)+k \mu \delta \alpha>\frac{\beta}{\mu}(\theta+\delta \alpha)^{2}, \\
& \text { (e) } \mu>\mu_{0}, \gamma>\gamma_{0} \text { and } \\
& \text { ( } \delta \alpha+\theta)(\alpha+\gamma)+k \mu \delta \alpha<\frac{\beta}{\mu}(\theta+\delta \alpha)^{2},
\end{aligned}
$$

where $\mu_{0}=\beta(\theta+\delta \alpha) /(\gamma+\delta \alpha)$ and $\gamma_{0}=\left((\delta \alpha+\theta)\left(\beta+\frac{1}{k} \alpha\right)-\frac{\beta}{k \mu}(\theta+\delta \alpha)^{2}\right) /(\mu-$ $\left.\frac{1}{k}(\delta \alpha+\theta)\right)$

Proof. Given $\left(\bar{n}_{1}, 0\right)$ is always an unstable equilibrium point, we derive the conditions that guarantee the equilibrium point with a non-zero fraction of negative individuals in (3.25) does not lie in the state space, $[0,1] \times[0,1]$. If $(\theta+\delta \alpha) /(k \mu)>1$, then this equilibrium does not lie in the state space. The condition (a) is equivalent to $(\theta+\delta \alpha) /(k \mu)>1$. The conditions (b) and (c) ensure that $\bar{u}$ in (3.25) is negative. Let $M$ and $D$ denote the numerator and denominator of $\bar{u}$ in (3.25), respectively. $\bar{u}$ in (3.25) is negative if and only if either $M>0$ and $D<0$, or $M<0$ and $D>0$. The inequalities $M<0$ and $D>0$ yield the condition (b), and $M>0$ and $D<0$ lead to the condition (c). If $\bar{u}$ in (3.25) is positive, $M>0$ and $D>0$, or $M<0$ and $D<0, \bar{u}$ being greater than one ensures that this equilibrium point does not lie in the state space. The inequalities $M>0$ and $D>0$, and $\bar{u}$ being greater than 1 yield the condition (d), and if $M<0$ and $D<0$, they yield the condition (e).

In the following lemma, we reduce the conditions in Theorem 3 for the special 
cases when the condition (a) in Theorem 2 is satisfied in order to guarantee a vanishing fraction of negative individuals in steady-state.

Lemma 2. The steady-state fraction of negative individuals will be 0 if $\mu<2 \beta(\theta+$ $\delta \alpha) /(k \beta+\alpha+\gamma)$ and $\alpha+\gamma>k \beta$.

Proof. Based on Theorem 2, if $\mu<2 \beta(\theta+\delta \alpha) /(k \beta+\alpha+\gamma)$, then $\left(\bar{n}_{2}, 0\right)$ is locally stable. Also, based on Theorem 3 , if $\mu<(\theta+\delta \alpha) / k$, there will be no equilibrium point with a non-zero fraction of negative individuals in the state space. Therefore, if

$$
\frac{2 \beta(\theta+\delta \alpha)}{k \beta+\alpha+\gamma}<\frac{1}{k}(\theta+\delta \alpha),
$$

and $\mu<2 \beta(\theta+\delta \alpha) /(k \beta+\alpha+\gamma)$, then the the condition (a) in Theorem 2 and the condition (a) in Theorem 3 are satisfied. The inequality in (3.27) simplifies to $\alpha+c>k \beta$. Therefore, if $\mu<2 \beta(\theta+\delta \alpha) /(k \beta+\alpha+\gamma)$ and $\alpha+c>k \beta$, then $\left(\bar{n}_{2}, 0\right)$ is locally stable and the equilibrium point in (3.25) with non-zero negative individuals does not lie in the state space. So, no matter what an initial state a trajectory begins with, it will always converge to either $\left(\bar{n}_{1}, 0\right)$ or $\left(\bar{n}_{2}, 0\right)$, where both have a zero fraction of negative individuals.

If $\left(\bar{n}_{2}, 0\right)$ is locally stable but not globally stable, then its region of attraction quantifies how robust this equilibrium point is with respect different initial states. The region of attraction for $\left(\bar{n}_{2}, 0\right)$ is the subset of the state space which trajectories initiating from, asymptotically converge to $\left(\bar{n}_{2}, 0\right)$. Determining the region of attraction is important since tells us how far the initial state can be from $\left(\bar{n}_{2}, 0\right)$ and still converge to $\left(\bar{n}_{2}, 0\right)$. In the following theorem, we estimate the region of attraction for $\left(\bar{n}_{2}, 0\right)$. 
Theorem 4. If $\left(\bar{n}_{2}, 0\right)$ is locally exponentially stable, trajectories converge to this equilibrium point if their initial states lie in the set $R_{D}$ defined as follows:

$$
R_{D}=[0,1]^{2} \cap \hat{R}_{D}
$$

where

$$
\hat{R}_{D}=\left\{(n, u) \mid p_{1}\left(n-\bar{n}_{2}\right)^{2}+2 p_{2}\left(n-\bar{n}_{2}\right) u+p_{4} u^{2}<\eta\right\}
$$

where

$$
\begin{aligned}
p_{1} & =-\frac{1}{2 A_{11}}, \\
x p_{2} & =\frac{A_{12}}{2 A_{11}\left(A_{11}+A_{22}\right)}, \text { and } \\
p_{4} & =\frac{-A_{12}^{2}}{2 A_{11} A_{22}\left(A_{11}+A_{22}\right)} .
\end{aligned}
$$

$A_{11}, A_{12}$, and $A_{22}$ are derived in (3.17). Also, $\eta$ is a positive number less than $\lambda_{\text {min }}(P) r^{2}$ where $r=1 /\left(f+\frac{1}{2} \sqrt{g^{2}+h^{2}}\right)$ and $P=\left[\begin{array}{ll}p_{1} & p_{2} \\ p_{2} & p_{4}\end{array}\right]$. And, $f=2 p_{1} k \beta$, $g=2\left(p_{1}(k \beta-k \mu)+p_{2}(k \beta+k \mu)\right), h=2\left(p_{2}(k \beta-k \mu)+p_{4} k \mu\right)$.

Proof. If $\left(\bar{n}_{2}, 0\right)$ is locally exponentially stable, the Jacobian matrix $A$ in (3.17), computed at $\left(\bar{n}_{2}, 0\right)$, is negative definite. We define $\hat{n}$ and $\hat{u}$ as follows:

$$
\begin{aligned}
& \hat{n}=n-\bar{n}_{2}, \\
& \hat{u}=u .
\end{aligned}
$$

Replacing $n$ and $u$ with $\hat{n}$ and $\hat{u}$ in (3.4), we can write

$$
\begin{aligned}
& \hat{n}^{\cdot}=k \beta \hat{n}^{2}+\left(2 k \beta n^{*}-(k \beta+\alpha+\gamma)\right) \hat{n}+(k \beta-k \mu) \hat{n} \hat{u}+ \\
& \quad\left((k \beta-k \mu) n^{*}+\theta-\gamma\right) \hat{n}, \\
& \hat{u}^{\cdot}=k \mu \hat{n} \hat{u}+\left(k \mu n^{*}-\theta-\delta \alpha\right) \hat{u} .
\end{aligned}
$$

The origin of the transformed dynamical system in (3.32) is corresponding to $\left(\bar{n}_{2}, 0\right)$ for the HOM-MEAN system in (3.4). We use Lyapunov functions to estimate the 
region of attraction for the origin of the transformed dynamical system. A Lyapunov function can be found by solving the Lyapunov equation

$$
P A+A^{T} P=-Q
$$

where $Q$ is a positive definite matrix. Taking $Q=I$, the unique solution is the positive definite matrix

$$
P=\left[\begin{array}{ll}
p_{1} & p_{2} \\
p_{2} & p_{4}
\end{array}\right],
$$

where

$$
\begin{aligned}
& p_{1}=-\frac{1}{2 A_{11}}, \\
& p_{2}=\frac{A_{12}}{2 A_{11}\left(A_{11}+A_{22}\right)}, \\
& p_{4}=\frac{-A_{12}^{2}}{2 A_{11} A_{22}\left(A_{11}+A_{22}\right)} .
\end{aligned}
$$

The quadratic function $V(\hat{n}, \hat{u})=p_{1} \hat{n}^{2}+2 p_{2} \hat{n} \hat{u}+p_{4} \hat{u}^{2}$ is a Lyapunov function for the transformed dynamical system in a certain neighborhood of the origin (Khalil (2000)). To find the region of attraction of the origin, we need to determine a domain $D$ around the origin where $V$ is positive definite and $V$ is negative definite. Since $P$ is positive definite, $D$ can be determined by studying negative definiteness of $V$. around the origin. We can write

$$
\begin{aligned}
& V \cdot(\hat{n}, \hat{u})=-\hat{n}^{2}+\left(2 p_{4} \hat{u}+2 p_{2} \hat{n}\right) k \mu \hat{n} \hat{u} \\
& -\hat{u}^{2}+\left(2 p_{1} \hat{n}+2 p_{2} \hat{u}\right)\left(k \beta \hat{n}^{2}+(k \beta-k \mu) \hat{n} \hat{u}\right) \\
& \quad=-\hat{n}^{2}-\hat{u}^{2}+f \hat{n}^{3}+g \hat{n}^{2} \hat{u}+h \hat{n} \hat{u}^{2},
\end{aligned}
$$

where $f=2 p_{1} k \beta, g=2\left(p_{1}(k \beta-k \mu)+p_{2}(k \beta+k \mu)\right)$, and $h=2\left(p_{2}(k \beta-k \mu)+p_{4} k \mu\right)$. 
Let $X:=\left[\begin{array}{ll}\hat{n} & \hat{u}\end{array}\right]^{T}$. Hence, it can be written

$$
\begin{aligned}
V^{\cdot} & =-\|X\|^{2}+f \hat{n}^{3}+\hat{n} \hat{u}(g \hat{n}+h \hat{u}) \\
& =-\|X\|^{2}+f \hat{n}^{3}+\hat{n} \hat{u}\left[\begin{array}{ll}
g & h
\end{array}\right] X \\
& \leqslant-\|X\|^{2}+f\|X\|^{3}+\frac{1}{2}\|X\|^{2} \sqrt{g^{2}+h^{2}}\|X\| \\
& \leqslant-\|X\|^{2}+\left(f+\frac{1}{2} \sqrt{g^{2}+h^{2}}\right)\|X\|^{3} .
\end{aligned}
$$

Therefore, $V$ is negative if $\|X\|<1 /\left(f+\frac{1}{2} \sqrt{g^{2}+h^{2}}\right)$. Let $r:=1 /\left(f+\frac{1}{2} \sqrt{g^{2}+h^{2}}\right)$. And, we have

$$
\eta<\min _{\|x\|=r} x^{T} P x=\lambda_{\min }(P) r^{2}
$$

Therefore, we can write

$$
R_{D}=[0,1]^{2} \cap \hat{R}_{D}
$$

where

$$
\hat{R}_{D}=\left\{(n, u) \mid p_{1}\left(n-n^{*}\right)^{2}+2 p_{2}\left(n-n^{*}\right) u+p_{4} u^{2}<\eta\right\}
$$

\subsection{Scale-Free Networks}

In this section, we use a mean-field approach to approximate the IN-STOCH system for scale-free networks by a dynamical system called the heterogeneous meanfield (HET-MEAN) system that is represented by a set of deterministic differential equations. Unlike exponential networks, the degree distributions of scale-free networks have strong fluctuations around their average node degrees. This characteristic of scale-free networks motivates a different mean-field analysis from the one for exponential networks, that takes into account the fraction of neutral, positive and negative nodes with given degree. Let $n_{k}(t), p_{k}(t)$ and $u_{k}(t)$ be the fraction of neutral, positive and negative individuals with node degree $k$ at time $t$. Therefore, 
$n_{k}(t)+p_{k}(t)+u_{k}(t)=1$. The HET-MEAN dynamical system is described with the following coupled differential equations in continuous-time:

$$
\begin{aligned}
& \dot{p}_{k}=k \beta \Phi(p) n_{k}+\alpha n_{k}-\gamma p_{k}+\delta \alpha u_{k}, \\
& \dot{n}_{k}=-k \beta \Phi(p) n_{k}-\alpha n_{k}+\gamma p_{k}-k \mu \Phi(u) n_{k}+\theta u_{k}, \\
& \dot{u}_{k}=k \mu \Phi(u) n_{k}-\theta u_{k}-\delta \alpha u_{k},
\end{aligned}
$$

where $\Phi(p)$ and $\Phi(u)$ denote the probabilities that any given edge is connected to a positive and a negative node, respectively. Therefore, $\Phi(p)+\Phi(u)+\Phi(n)=1$, where $\Phi(n)$ is the probability that any given edge is connected to a neutral node. We can write

$$
\Phi(x)=\frac{1}{\langle k\rangle} \sum k P(k) x_{k},
$$

where $x$ can be either $p, n$ or $u$, and $P$ denotes the degree distributions of influence networks. Also, average node degree is denoted by $\langle k\rangle$. The HET-MEAN system assumes the independence between states of individuals. Using $n_{k}+p_{k}+u_{k}=1$ reduces (3.41) to the following system:

$$
\begin{aligned}
\dot{n}_{k} & =k(\beta-\mu) \Phi(u) n_{k}+k \beta \Phi(n) n_{k}-(k \beta+\alpha+\gamma) n_{k} \\
& +(\theta-\gamma) u_{k}+\gamma \\
\dot{u}_{k} & =k \mu \Phi(u) n_{k}-(\theta+\delta \alpha) u_{k} .
\end{aligned}
$$

The variables of the reduced mean-field dynamical system in (3.43) are $\left\{n_{k}\right\}$ and $\left\{u_{k}\right\}$. Therefore, the dimension of this dynamical system state space is $2 K$, where $K$ is the number of different degrees of the nodes. Imposing the stationary conditions, $\dot{n}_{k}=0$ and $\dot{u}_{k}=0$, we find the equilibrium points. Let $\left\{\left(\bar{n}_{k}, \bar{u}_{k}\right)\right\}$ be an equilibrium point of this dynamical system. From $\dot{u}_{k}=0$, we see that either $\bar{u}_{k}=0$ or

$$
\bar{n}_{k}=\frac{\theta+\delta \alpha}{k \mu \bar{\Phi}(u)} \bar{u}_{k},
$$


where $\bar{\Phi}(u)$ and $\bar{\Phi}(n)$ denote the values of $\Phi(u)$ and $\Phi(n)$ that satisfy stationary conditions, respectively. For the equilibrium point with no negative individuals, $\bar{u}_{k}=$ 0 , we have $\bar{\Phi}(u)=0$. Moreover, replacing $u_{k}=0$ in $n_{k}=0$, we have

$$
\bar{n}_{k}=\frac{\gamma}{k \beta(1-\bar{\Phi}(n))+\alpha+\gamma} .
$$

In the following Lemma, we show that $\left\{\left(\bar{u}_{k}=0, \bar{n}_{k}=\gamma /(k \beta(1-\bar{\Phi}(n))+\alpha+\gamma)\right)\right\}$ is always an equilibrium point for the HET-MEAN system.

Lemma 3. The HET-MEAN system has exactly one equilibrium point with no negative nodes given by

$$
\left\{\left(\bar{u}_{k}=0, \bar{n}_{k}=\frac{\gamma}{k \beta(1-\bar{\Phi}(n))+\alpha+\gamma}\right)\right\}
$$

Proof. Using the stationary conditions, we showed that the only candidate for equilibrium points with no negative individuals is (3.46). This point will be an equilibrium point if and only if it lies in the state space of the dynamical system. That is $\forall k$,

$$
0 \leqslant \bar{n}_{k} \leqslant 1
$$

Equation (3.47) is equivalent to $0 \leqslant \bar{\Phi}(n) \leqslant 1$. Now, we show that $0 \leqslant \bar{\Phi}(n) \leqslant 1$ always holds. We have

$$
\begin{aligned}
\bar{\Phi}(n) & =\frac{1}{\langle k\rangle} \sum k \bar{n}_{k} P(k) \\
& =\frac{1}{\langle k\rangle} \sum \frac{\gamma k P(k)}{k \beta(1-\bar{\Phi}(n))+\alpha+\gamma}
\end{aligned}
$$

Let $g(\bar{\Phi}(n)):=\sum \gamma k P(k) /(k \beta(1-\bar{\Phi}(n))+\alpha+\gamma) /\langle k\rangle$. Therefore, $0 \leqslant \bar{\Phi}(n) \leqslant 1$ if and only if the equation $\bar{\Phi}(n)=g(\bar{\Phi}(n))$ has a solution for $\bar{\Phi}(n)$ in $[0,1]$. We have

$$
\begin{aligned}
& g(0)=\frac{1}{\langle k\rangle} \sum \frac{k P(k)}{1+\frac{1}{c}(k \beta+\alpha)} \\
& g(1)=\frac{\gamma}{\gamma+\alpha} .
\end{aligned}
$$


We have $0 \leqslant g(0) \leqslant 1$ and $0 \leqslant g(1) \leqslant 1$. Therefore, $\bar{\Phi}(n)=g(\bar{\Phi}(n))$ has a solution in $[0,1]$.

One can examine the stability of the equilibrium point in (3.46) by computing the eigenvalues of $2 K \times 2 K$ Jacobian matrix, at this equilibrium point, of the dynamical system in (3.43). The partial derivatives of the system in (3.43), computed at the equilibrium point in (3.46), can be written as

$$
\begin{aligned}
& \frac{\partial \dot{n}_{k}}{\partial n_{k}}=k \beta \bar{\Phi}(n)+\frac{k^{2}}{\langle k\rangle} \beta \bar{n}_{k} P(k)-(k \beta+\alpha+\gamma) \\
& \forall j \neq k, \frac{\partial \dot{n}_{k}}{\partial n_{j}}=\frac{k j}{\langle k\rangle} \beta \bar{n}_{k} P(j) \\
& \frac{\partial \dot{n}_{k}}{\partial u_{k}}=\frac{k^{2}}{\langle k\rangle}(\beta-\mu) \bar{n}_{k} P(k)+\theta-\gamma \\
& \forall j \neq k, \frac{\partial \dot{n}_{k}}{\partial u_{j}}=\frac{k j}{\langle k\rangle}(\beta-\mu) \bar{n}_{k} P(j) \\
& \frac{\partial \dot{u}_{k}}{\partial n_{k}}=0, \forall j \neq k, \frac{\partial u_{k}}{\partial n_{j}}=0 \\
& \frac{\partial \dot{u}_{k}}{\partial u_{k}}=\frac{k^{2}}{\langle k\rangle} \mu \bar{n}_{k} P(k)-(\theta+\delta \alpha) \\
& \forall j \neq k, \frac{\partial \dot{u}_{k}}{\partial u_{j}}=\frac{k j}{\langle k\rangle} \mu \bar{n}_{k} P(j) .
\end{aligned}
$$

Let $J_{\bar{n}}$ denote the $K \times K$ sub-block corresponding to $\partial \dot{n}_{k} / \partial n_{k}$ and $\partial \dot{n}_{k} / \partial n_{j}$ of the Jacobian matrix computed at the equilibrium point in (3.46). Also, the $K \times K$ subblock corresponding to $\partial \dot{u}_{k} / \partial u_{k}$ and $\partial \dot{u}_{k} / \partial u_{j}$ of the Jacobian matrix is denoted by $J_{\bar{u}}$.

Theorem 5. The equilibrium point with no negative individuals in (3.46) is locally exponentially stable if and only if the real parts of all eigenvalues of $J_{\bar{n}}$ and $J_{\bar{u}}$ are negative. 
Proof. The equilibrium point in (3.46) is locally exponentially stable if and only if the real parts of all eigenvalues of the Jacobian matrix, computed at this equilibrium point, are negative. Because $\partial \dot{u}_{k} / \partial n_{j}=0$ at the equilibrium point in (3.46), the eigenvalues of the Jacobian matrix are equal to union of the eigenvalues of $J_{\bar{n}}$ and $J_{\bar{u}}$. Therefore, this equilibrium point is locally exponentially stable if and only if the real parts of all eigenvalues of $J_{\bar{u}}$ and $J_{\bar{n}}$ are negative.

For the other equilibrium point corresponding to $\bar{n}_{k}=\bar{u}_{k}(\theta+\delta \alpha) /(k \mu \bar{\Phi}(u))$, replacing $\bar{n}_{k}$ by $\bar{u}_{k}(\theta+\delta \alpha) /(k \mu \bar{\Phi}(u))$ in $n=0$, we have

$$
\bar{u}_{k}=\frac{\gamma k \mu \bar{\Phi}(u)}{I+(\gamma-\theta) k \mu \bar{\Phi}(u)},
$$

where $I=(\theta+\delta \alpha)(k(\mu-\beta) \bar{\Phi}(u)+k \beta(1-\bar{\Phi}(n))+\alpha+\gamma)$. Also, because $\bar{n}_{k}=$ $\bar{u}_{k}(\theta+\delta \alpha) /(k \mu \bar{\Phi}(u))$, we have

$$
\bar{\Phi}(n)=\frac{\theta+\delta \alpha}{k \mu} .
$$

\subsubsection{Barabasi-Albert Networks}

In this section, we apply our theoretical results for the HET-MEAN system to Barabasi-Albert (BA) networks, which are examples of scale-free networks (Barabasi and Albert (1999)). The underlying idea behind the BA networks is that individuals tend to make connections with individuals who are more well-known and who have a larger number of existing connections. This is an intuitive phenomenon that is observed on social networks. To construct a BA network, we start from a small number $m_{0}$ of disconnected nodes; every new node forms $m$ edges to existing nodes such that the probability of a new edge to be connected to an old node is proportional to the degree of old node (Barabasi and Albert (1999)). The constructed network has the 
following degree distribution

$$
P(k)=\left\{\begin{array}{ll}
2 m^{2} k^{-3}, & \text { if } k \geqslant m \\
0, & \text { otherwise }
\end{array},\right.
$$

with average node degree $\langle k\rangle=2 m$ (Barabasi and Albert (1999)).

For the equilibrium point with no negative individuals in (3.46), we have $\bar{n}_{k}=$ $\gamma /(k \beta(1-\bar{\Phi}(n))+\alpha+\gamma))$. In order to compute the steady-state fraction of neutral nodes $\bar{n}=\sum P(k) \bar{n}_{k}$, first, we need to compute $\bar{\Phi}(n)$. We can calculate $\bar{\Phi}(n)$ using a self-consistency equation that is derived from the fact that $\bar{n}_{k}$ is also a function of $\bar{\Phi}(n)$. We approximate the sum in (3.54) by an integral in order to derive the self-consistency equation. Therefore, we have

$$
\begin{aligned}
\bar{\Phi}(n) & =\frac{1}{\langle k\rangle} \sum_{k=m}^{\infty} k P(k) \bar{n}_{k} \\
& \approx \frac{1}{2 m} \int_{m}^{\infty} \frac{2 m^{2} k^{-2} \gamma}{k \beta(1-\bar{\Phi}(n))+\alpha+\gamma} d k .
\end{aligned}
$$

Computing the integral in (3.54), we derive the following self-consistency equation for $\bar{\Phi}(n)$ :

$$
\begin{aligned}
& \ln \left(1+\frac{\alpha+\gamma}{m \beta(1-\bar{\Phi}(n))}\right) \\
& =\frac{\alpha+\gamma}{m \beta \gamma}\left(\gamma-\alpha \frac{\bar{\Phi}(n)}{1-\bar{\Phi}(n)}\right)
\end{aligned}
$$

where $\bar{\Phi}(n)$ can be found numerically. Also, using the integral approximation, we have

$$
\begin{aligned}
\bar{n} & =\sum_{k=m}^{\infty} P(k) \bar{n}_{k} \approx \int_{m}^{\infty} 2 m^{2} k^{-3} \frac{\gamma}{k \beta(1-\bar{\Phi}(n))+\alpha+\gamma} d k \\
& =\frac{2 m^{2} \gamma \beta^{2}(1-\bar{\Phi}(n))^{2}}{(\alpha+\gamma)^{3}} \ln \left(1+\frac{\alpha+\gamma}{m \beta(1-\bar{\Phi}(n))}\right) \\
& +2 m^{2} \gamma\left(\frac{\beta(1-\bar{\Phi}(n))}{(\alpha+\gamma)^{2} m}-\frac{1}{2(\alpha+\gamma) m^{2}}\right) .
\end{aligned}
$$


For the other equilibrium point corresponding to $\bar{n}_{k}=\bar{u}_{k}(\theta+\delta \alpha) /(k \mu \bar{\Phi}(u))$, first we calculate $\bar{\Phi}(u)$ through a self-consistency equation using the fact that $\bar{u}_{k}$ is a function of $\bar{\Phi}(u)$. Employing the integral approximation, we can write

$$
\begin{aligned}
\bar{\Phi}(u) & =\frac{1}{\langle k\rangle} \sum_{k=m}^{\infty} k P(k) \bar{u}_{k} \\
& \approx \frac{1}{2 m} \int_{m}^{\infty} 2 m^{2} k^{-2} \bar{u}_{k} d k \\
& =\bar{\Phi}(u) \frac{m s f}{g} \ln \left(1+\frac{g}{m(f \bar{\Phi}(u)+h)}\right),
\end{aligned}
$$

where $s=\gamma \mu^{2} / f ; f=\mu((\mu-\beta)(\theta+\delta \alpha)+(\gamma-\theta)) ; g=(\theta+\delta \alpha)(\beta(\delta \alpha-\theta)+\mu(\alpha+\gamma))$; $h=\beta \mu(\theta+\delta \alpha)$. Using the self-consistency equation in (3.57), we can derive explicitly $\bar{\Phi}(u)$ as follows:

$$
\bar{\Phi}(u)=\frac{g}{m f}\left(e^{\frac{g}{m \gamma \mu^{2}}}-1\right)^{-1}-\frac{h}{f} .
$$

Finally, using the integral approximation, we can derive the fraction of negative individuals as

$$
\bar{u}=\sum_{k=m}^{\infty} P(k) \bar{u}_{k} \approx \int_{m}^{\infty} 2 m^{2} k^{-3} \bar{u}_{k},
$$

where $\bar{u}_{k}$ is derived in (3.51). Computing the integral in (3.59), we have

$$
\bar{u}=H\left(1-\frac{m}{g}(f \bar{\Phi}(u)+h) \ln \left(1+\frac{g}{(f \bar{\Phi}(u)+h) m}\right)\right),
$$

where $H=2 m s f \bar{\Phi}(u) / g$.

However, Theorem 5 can be used to determine the stability of the equilibrium point with no negative individuals in (3.46) for the BA networks, here, we derive an alternative criteria to examine the stability of this equilibrium point. Given $\bar{\Phi}(u)$ is the probability that any given edge is connected to a negative individual, having no negative node in steady state is equivalent to $\bar{\Phi}(u) \leqslant 0$. 


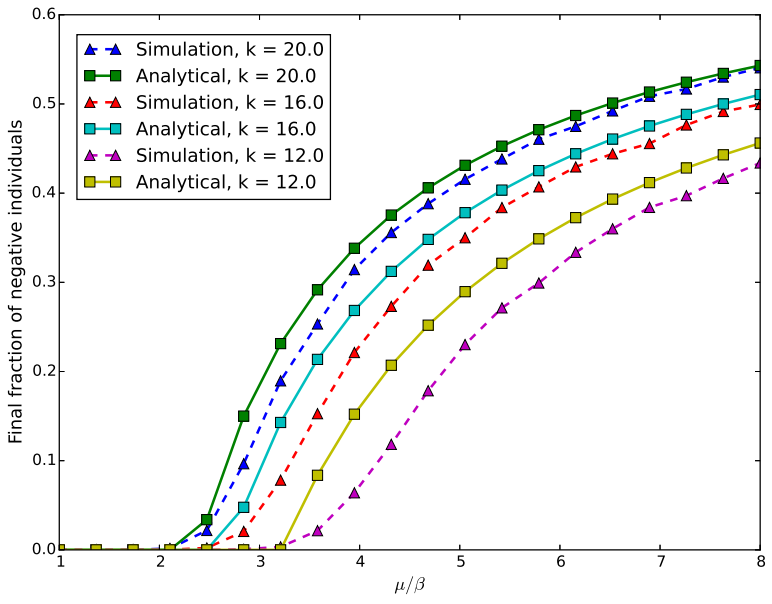

FiguRE 3.2: Final fraction of negative individuals for WS networks as a function of $\mu / \beta$. Solid lines are theoretical predictions. Dashed lines are simulation results. Parameter values: $\theta=0.01, \beta=0.001, \alpha=0.01, \delta=0.4$, and $\gamma=0.01$.

Lemma 4. For the BA networks, asymptotic fraction of negative individuals will be 0 if

$$
e^{\frac{g}{m c \mu^{2}}}-1 \geqslant \frac{1}{m}\left(\frac{1}{\mu}(\delta \alpha-\theta)+\frac{1}{\beta}(\alpha+c)\right) .
$$

Proof. The inequality in (3.61) is equivalent to $\bar{\Phi}(u) \leqslant 0$.

The main advantage of Lemma 4 over Theorem 5 to check whether the fraction of negative individuals is equal 0 for the BA networks, is its lower computational cost. It is because for Theorem 5 , we need to compute the eigenvalues of two $K \times K$ matrices.

\subsection{Simulation Results}

In this section, we compare the analytical approximations by the HEM-MEAN and HET-MEAN systems with the simulation results of the IN-STOCH system on random regular, Watts-Strogatz (WS) and BA networks as well as the ego-Facebook network (Leskovec and Mcauley (2012)). In particular, we compare the analytical 


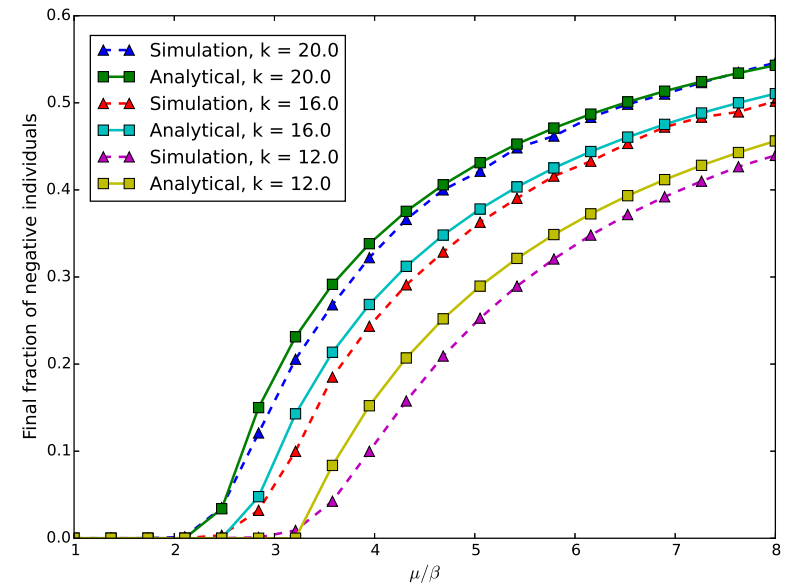

FiguRE 3.3: Final fraction of negative individuals for random regular networks as a function of $\mu / \beta$. Solid lines are theoretical predictions. Dashed lines are simulation results. Parameter values: $\theta=0.01, \beta=0.001, \alpha=0.01, \delta=0.4$, and $\gamma=0.01$.

approximations for the steady-state fraction of negative individuals with the results of simulating the IN-STOCH system over the influence networks. For WS and BA networks, the analytical approximations are based on the results of Theorems 2 and 3 in combination with the derived equilibrium point with a non-zero fraction of negative individuals in (3.25). If either Theorem 2 or Theorem 3 is satisfied, then the analytical approximation for steady-state fraction of negative individuals is equal to 0 , otherwise, it is equal to (3.25).

In the first experiment, simulations are performed using randomly generated WS networks with $N=10000$ nodes. WS networks are used as reference point for homogeneous networks that model small-world networks. WS networks are shown to be good candidates to model real-world networks in the context of social networks (Watts and Strogatz (1998)). Small-world networks are networks with short average path lengths and high clustering. To construct a WS network, we start from a ring of $N$ nodes. Choosing an even number $k$, each node is connected with its $k / 2$ nearest clockwise neighbors and $k / 2$ nearest counter clockwise neighbors. Then, with 
probability $p$, every link connected to a clockwise neighbor is rewired to a randomly chosen node. As a result of this process, a WS network with the average node degree $k$ is created. One of the important characteristics of these network is their small fluctuations in connectivity due to their exponential degree distributions.

Initially, $60 \%$ of nodes are neutral, $10 \%$ positive and $30 \%$ negative. Then, we iterate the rules of the IN-STOCH system for 2000 steps. Three different ensembles of WS networks are studied with average node degrees of $k=12,16$ and 20, and with probability of rewiring $p=0.2$. In Figure 3.2 , both analytical and simulation results of final fraction of negative individuals as a function of $\mu / \beta$ in the WS networks are presented. The final faction of negative individuals in the steady-state is averaged over 10 different realizations of WS networks for each case. The analytical approximations of Theorems 2 and 3, based on the HOM-MEAN system, are in total agreement with the simulation results of the IN-STOCH system; They correctly predict when final fractions of negative individuals are 0 . When neither Theorem 2 nor Theorem 3 hold, the analytical approximation for the steady-state fraction of negative individuals by (3.25) is in good agreement with the simulation results. This agreement becomes stronger by increasing the average node degree. Also, Theorem 2 predicts that the regime 1 is active for all the three cases, which is confirmed by simulation results. The negative-free threshold decreases by increasing the average node degree. It shows that for the given scenario, increasing the average node degree helps the word-of-mouth process by negative individuals more than the word-of-mouth process by positive individuals.

In the second experiment, simulations are performed in random regular networks with $N=10000$ nodes. Random regular networks are similar to regular networks in the sense that all nodes have identical node degrees. However, neighbors of each nodes is chosen randomly. Initial percentage of neutral, positive and negative nodes are 60, 10 and 30, respectively. Iterating the rules of the IN-STOCH system is done 

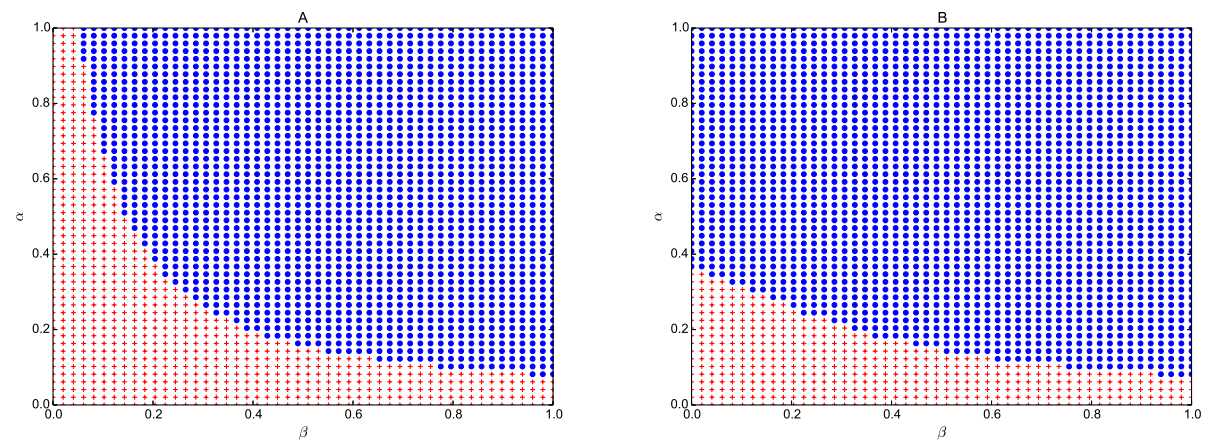

Figure 3.4: Comparison between viral diffusion and media influence over the WS network (Parameter values: $\theta=0.0001, \delta=0.05, \gamma=0.5$, and $\mu=0.008$ ). (A) $k=40(\mathrm{~B}) k=4$.

over three ensembles of random regular networks with node degrees of $k=12,16$ and 20 for 2000 steps. In Figure 3.3, we plot the analytical approximations and the simulation results of final fractions of negative individuals versus $\mu / \beta$. The plotted simulation results for each node degree are resultant of averaging the simulation results of 10 different realization of random regular networks. Similar to WS networks, the simulation results are in total agreement with the analytical approximations of Theorems 2 and 3. And, when neither of these two theorems hold, the steady-state fractions of negative individuals predicted by (3.25) are in good agreement with the simulation results. Following the same trend as WS networks, this agreement becomes stronger by increasing the node degree. However, for all three values of node degrees, (3.25) predicts more accurately the steady-state fraction of negative individuals for the random regular networks compared to the WS networks. This is because the HOM-MEAN system assumes that all nodes have identical node degrees, which is true for random regular networks, but not for WS networks. Similar to WS networks, the negative-free threshold decreases by increasing the average node degree.

The HOM-MEAN system can be used to compare the effectiveness of viral diffusion and media influence under the IN-STOCH system over exponential influence 


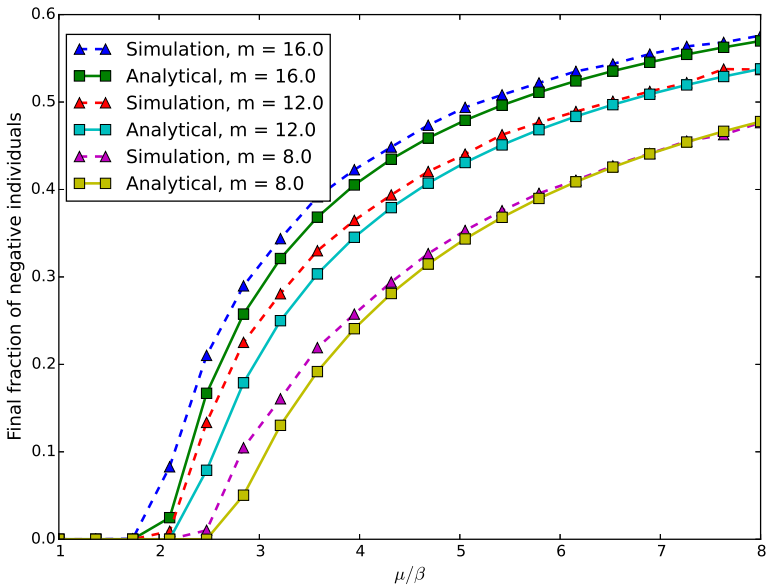

FIGURE 3.5: Final fraction of negative individuals for BA networks as a function of $\mu / \beta$. Solid lines are theoretical predictions. Dashed lines are simulation results. Parameter values: $\theta=0.01, \beta=0.001, \alpha=0.01, \delta=0.4$, and $\gamma=0.01$.

networks. In the IN-STOCH system, $\beta / \alpha$ represents the ratio of investment of intervening agencies on viral diffusion over media influence. Every intervening strategy is modeled as a combination of viral diffusion and media influence, and is represented by a pair $(\beta, \alpha)$. Therefore, the set of $\{(\beta, \alpha) \in[0,1] \times[0,1]\}$ spans the space of different intervening strategies for a given scenario. A scenario is fully characterized by $\gamma, \theta, \delta, \mu$ and the influence network. We partition the space of intervening strategies into two subsets $\Omega$ and $\Psi$ based on steady-state fraction of negative individuals using the approximations by the HOM-MEAN system. $\Omega$ is the subset of those intervening strategies whose corresponding steady-state fractions of negative individuals are zero. And, the subset $\Psi$ consists of those intervening strategies with non-zero steady-state fractions of negative individuals.

Figure 3.4 depicts subsets $\Omega$ and $\Psi$ intervening strategies for two scenarios differing only in average node degree. Blue circles represent intervening strategies belonging to $\Omega$, and intervening strategies in $\Psi$ are represented by red circles. For scenario in (A), average node degree is 40 , and is 4 for (B). As we expect, subsets $\Omega$ and $\Psi$ 


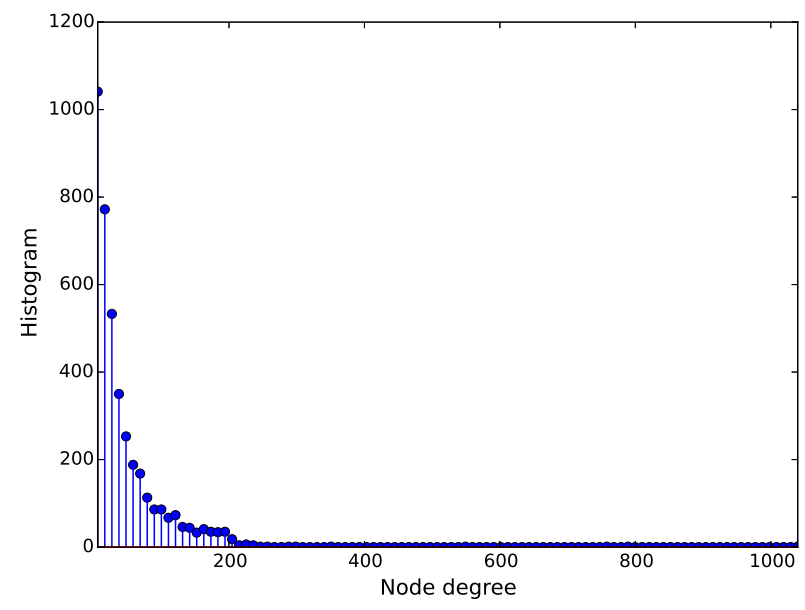

FiguRE 3.6: Histogram of node degrees of the ego-Facebook network.

differ for these two scenarios, and their main difference is the region in the top left corresponding to small values of $\beta$ and large values of $\alpha$. The intervening agencies in this region have vanishing fractions of negative individuals in (B), and non-zero steady-state fractions of negative individuals in (A). It is because for this scenario, increasing average node degree contributes more to the word-of-mouth process by negative individuals rather than positive individuals. So, in (B), for small values of $\beta$, strong media influence, large values of $\alpha$, can overcome the negative impact of the word-of-mouth process by negative individuals. However, in (A), it does not happen because increasing average node degree helps the word-of-mouth process by negative individuals more than positive individuals such that strong media influence cannot overcome the impact of negative individuals anymore. We can conclude that in (A), using any of the two viral diffusion and media influence is not advantageous over the other one. However, in (B), media influence is recommended over viral diffusion. For example, if $\alpha>.4$, then it is guaranteed that intervening strategies will result in zero steady-state fractions of negative individuals in (B).

We validate the approximations by the HET-MEAN system through simulations 
of the IN-STOCH system over the BA networks with $N=1000$ nodes. Three different ensembles of BA networks with the parameters of $m=8,12$ and 16 are randomly generated. For the all generated BA networks, $m_{0}$ is equal to $m$. Simulations start with 60 percent of individuals being in the neutral state, 10 percent in the positive state and 30 percent in the negative state. For all cases, the simulation length is equal to 5000 steps. Figure 3.5 plots the approximations of steady-state fractions of negative individuals by the HET-MEAN system and the simulation results versus $\mu / \beta$. The analytical approximations are computed as follows: If the condition (3.61) in Lemma 4 is satisfied, then the final fraction of the negative individuals is equal to 0, otherwise (3.60) is used to compute the final fraction of the negative individuals. The plotted simulation results for each value of $m$ are resultant of averaging the simulation results of 10 different realization of the BA networks. We observe that the analytical approximations in a good agreement with the simulation results.

Moreover, the IN-STOCH system is simulated over the ego-Facebook network (Leskovec and Mcauley (2012)). This undirected network consists of 4039 nodes and 88234 edges with average node degree 43.7 and standard deviation 52.4; It was generated by using a Facebook app based on friends lists. Histogram of node degrees of the ego-Facebook network which is plotted in Figure 3.6, shows that its node degree distribution follows a power law distribution. The simulation starts with $60 \%$ of nodes being in the neutral state, $10 \%$ positive and $30 \%$ negative, and is performed for 10000 steps by iterating the rules of the IN-STOCH system. Figure 3.7 depicts the analytical approximations and simulation results. The analytical approximations are obtained based on the differential equations describing the HETMEAN system in (3.43) as follows: First, we compute the exact degree distribution of the ego-Facebook network. Then, we simulate the dynamical system in (3.43) till its state evolution reaches the steady-state. Finally, we report the fraction of negative individuals at the steady-state. We observe that the analytical approximations by 


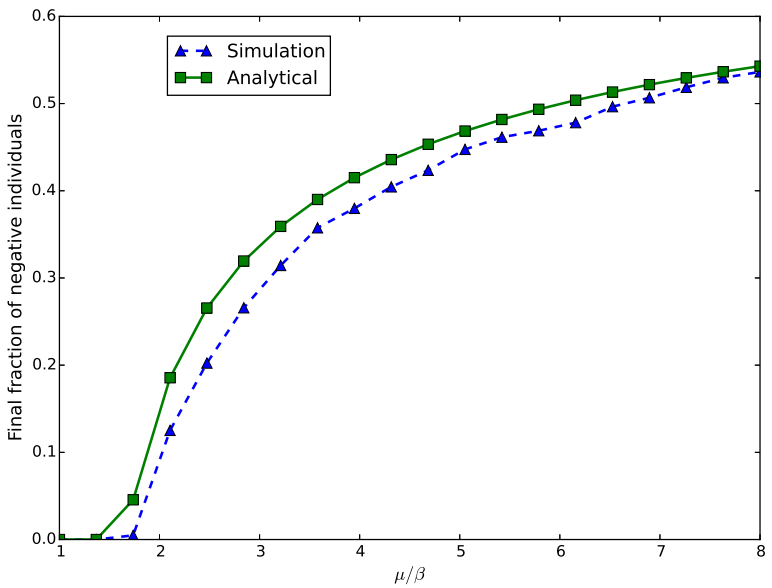

FIGURE 3.7: Final fraction of negative individuals in the ego-Facebook network as a function of $\mu / \beta$. The solid line is the theoretical predictions. The dashed line is the simulation results. Parameter values: $\theta=0.01, \beta=0.001, \alpha=0.01, \delta=0.4$, and $\gamma=0.01$.

the HET-MEAN system for this real-world network are in a good agreement with the simulation results of the IN-STOCH system.

\subsection{Conclusion}

In this chpater, we propose the IN-STOCH system to model the adoption of polarized beliefs in influence networks, governed by both viral diffusion and media influence. We study the intertwined dynamics of these two different forms of influence, and how they interact over influence networks. Using a mean-field approach, we derive the HOM-MEAN system to approximate the IN-STOCH system for exponential networks. Based one the stability of the equilibrium points of the HOM-MEAN system, we derive the conditions to guarantee the convergence of the fraction of negative individuals to 0. Also, we derive the HET-MEAN system to approximate the IN-STOCH system for scale-free networks and show that the HET-MEAN system always has exactly one equilibrium point with no negative individuals. In particular, we apply our results for the HET-MEAN system to the BA networks and compute a simplified 
condition for the stability of the equilibrium point with no negative individuals for the BA networks. Finally, we validate our theoretical approximations by simulation the IN-STOCH system over WS, random regular, BA and the ego-Facebook networks.

Simulation results show that the HEM-MEAN system can accurately predict the convergence to zero fo the fraction of negative individuals for WS and random regular networks. For cases where the final fractions of negative individuals are not 0 , the analytical approximation given by the HEM-MEAN system is in good agreement with simulation results for WS and random regular networks. This agreement also improves when the average node degrees increase. In general, the analytical approximations show a higher accuracy for random regular networks compared to WS networks since random regular networks satisfy the underlying assumption of the HEM-MEAN system, all nodes have the same number of neighbors.

Moreover, using analytical approximations for the steady-state fractions of negative individuals, we partition the space of intervening strategies into two subsets of strategies with a vanishing fraction of negative individuals and non-zero fraction of negative individuals. The space of intervening strategies is spanned by values of $\alpha$ and $\beta$, which represent the investments of intervening agencies in media influence and viral diffusion, respectively. We show that the separating curves of these two subsets for fixed set of parameters changes drastically according to the average node degree.

Finally, the analytical approximations for scale-free networks by the HET-MEAN system are validated by simulations of the IN-STOCH system over the BA networks. It is shown that the analytical results obtained by studying the equilibrium point of the HET-MEAN system are in a good agreement with the simulation results. In particular, the HET-MEAN system can accurately predict the cases where the steadystate fractions of negative individuals are equal to 0 . Also, for the ego-Facebook, the simulation results of the IN-STOCH system are in a good agreement with the ana- 
lytical approximations of the HET-MEAN system using the exact computed degree distribution of the ego-Facebook network. This shows that the HET-MEAN system can be used to approximate the IN-STOCH system for the real-world networks. 
4

\section{Modeling the Dynamics of Polarized Beliefs in Networks Governed by Viral Diffusion and Media Influence Using the Potts Model}

\subsection{Introduction}

In this chapter, we study the propagation of polarized beliefs in social networks using a mathematical model that is different from the one used in Chapter 3. The new model treats individuals as a system of interacting particles, each having three states. This is a special case of the Potts model from statistical physics (Potts (1952)).

We propose an adoption model where at any given time $t$, each node is in one of the three states: neutral $(\mathrm{N})$, positive $(\mathrm{P})$ and negative $(\mathrm{U})$. This model describes $M$ individuals $\left(x_{1}(t), \ldots, x_{M}(t)\right) \equiv \underline{x}(t)$ taking values in a finite alphabet $X=$ $\{N, P, U\}$. The interactions among individuals are determined by their underlying bidirectional influence networks. In particular, the set of undirected edges of a given network is denoted by $A$. If nodes $i$ and $j$ are connected, then $\{i, j\} \in A$.

The viral influence among individuals is modeled by a pairwise energy function 
$E_{\{i, j\}}\left(x_{i}, x_{j}\right)$ for every $\{i, j\} \in A$ as follows:

$$
E_{\{i, j\}}\left(x_{i}, x_{j}\right)=J_{N P} \delta_{\left\{x_{i}, x_{j}\right\}}(N, P)+J_{N U} \delta_{\left\{x_{i}, x_{j}\right\}}(N, U)+J_{P U} \delta_{\left\{x_{i}, x_{j}\right\}}(P, U),
$$

where $\delta_{\left\{x_{i}, x_{j}\right\}}(r, e)$ is an indicator function that is equal to one iff $x_{i}=r$ and $x_{j}=e$, or $x_{j}=r$ and $x_{i}=e$. The media influence is modeled by an individual energy function $E_{i}\left(x_{i}\right)$ for every node as follows:

$$
E_{i}\left(x_{i}\right)=h_{N} \delta_{x_{i}}(N)+h_{P} \delta_{x_{i}}(P)+h_{U} \delta_{x_{i}}(U),
$$

where $\delta_{x_{i}}(r)$ is an indicator function that is equal to one iff $x_{i}=r$. Putting together the pairwise and individual energy functions, one can write the Hamiltonian governing the system as

$$
H(\underline{x})=\sum_{\{i, j\} \in A} E_{\{i, j\}}\left(x_{i}, x_{j}\right)+\sum_{i=1}^{M} E_{i}\left(x_{i}\right) .
$$

Using the Hamiltonian function in (4.3), we can write the Boltzmann distribution as

$$
\mu(\underline{x})=\frac{1}{Z} e^{-\beta H(\underline{x})},
$$

where $Z=\sum_{\underline{x}} e^{-\beta H(\underline{x})}$ denotes the partition function and $\beta$ denotes the reciprocal of the thermodynamic temperature of the system.

The Boltzmann distribution in (4.4) also can be written in terms of factor functions. The following factor functions are defined in terms of energy functions in (4.1) and (4.2) as follows: $\psi_{\{i, j\}}\left(x_{i}, x_{j}\right)=\exp \left(-\beta E_{\{i, j\}}\left(x_{i}, x_{j}\right)\right)$ and $\psi_{i}\left(x_{i}\right)=\exp \left(-\beta E_{i}\left(x_{i}\right)\right)$. Therefore, we can write

$$
\mu(\underline{x})=\frac{1}{Z} \prod_{\{i, j\} \in A} \psi_{\{i, j\}}\left(x_{i}, x_{j}\right) \prod_{i=1}^{M} \psi_{i}\left(x_{i}\right),
$$

where $Z$ is the partition function. Since direct computation of $Z$ requires exponential complexity, a variety of other methods have been proposed for exact computation 
or estimation of the distribution in (4.5). In this project, we employ two different methods, the Metropolis-Hastings algorithm and the belief propagation algorithm, to estimate this distribution.

We use the Metropolis-Hastings (MH) algorithm (Hastings (1970)) based on the Hamiltonian in Eq. (4.3). At time $t$, we select a node $i$ at random. Then, we change its state from $x_{i}(t)$ to $\hat{x}_{i}$ with the acceptance probability

$$
A\left(x_{i}(t) \rightarrow \hat{x}_{i}\right)=\min (1, \exp (-\beta(H(\underline{\hat{x}})-H(\underline{x})))
$$

where $\underline{\hat{x}}=\left(x_{1}(t), \ldots, \hat{x}_{i}, \ldots, x_{M}(t)\right)$. We continue sampling until the estimated nodetype marginal distribution converges to its stationary distribution. This operation defines the Markov chain dynamics that we assume for the stochastic system. The convergence to the node-type marginal stationary distribution is checked by running 3 independent chains of the MH algorithm and examining if the estimated node-type marginal stationary distributions match closely. In the following section, we describe the belief propagation algorithm computed for the distribution in (4.5).

\subsection{Estimation of Node-Type Marginal Distributions using Belief Prop- agation}

In this section, we use belief propagation (BP) algorithm to estimate the node-type marginal distribution. The BP algorithm computes exact node-type marginal distributions for tree-graphical models, models with tree factor graphs, and an estimation for loopy graphical models.

In Figure 4.1, a snippet of the factor graph is shown with depth 1 rooted at $x_{1}$ of factor graph of the factorized distribution in (4.5) for regular networks with node degree 3 is plotted. The factor node corresponding to the media influence, $\psi_{1}$ is only

connected to $x_{1}$ whereas the factor nodes corresponding to the viral influence have degree 2 and connect $x_{1}$ to its neighbours. 


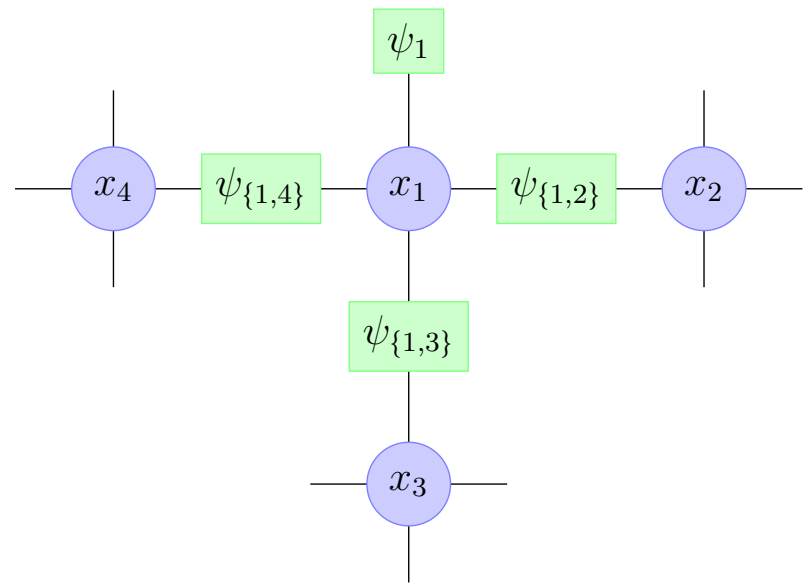

FiguRE 4.1: Subgraph with depth 1 of the factor graph of the proposed model for regular networks with node degree 3.

\subsection{Belief Propagation Equations}

In this section, we derive the $\mathrm{BP}$ equations to estimate the node-type marginal distributions of variables nodes. The BP message updates below follow directly from applying the BP algorithm to the factor graph defined by (4.5) (Mezard and Montanari (2009)). The BP algorithm is based on exchange of messages between variable and factor nodes along the edges of the factor graph. The message from check node $\psi_{\{i, j\}}$ to variable node $i$ is denoted by $\hat{\nu}_{\{i, j\} \rightarrow i}$ and the message from the variable node $i$ to check node $\psi_{\{i, j\}}$ is denoted by $\nu_{i \rightarrow\{i, j\}}$. Also, $\hat{\nu}_{\{i\} \rightarrow i}$ denotes the message from check node $\psi_{\{i\}}$ to variable node $i$ and the message from variable node $i$ to check node $\psi_{\{i\}}$ is denoted by $\nu_{i \rightarrow\{i\}}$.

All the messages have three components denoted by $[\ldots]_{s}$, where $s \in\{N, P, U\}$. The update for $\hat{\nu}_{\{i\} \rightarrow i}^{t}$ is given by

$$
\begin{aligned}
& {\left[\hat{\nu}_{\{i\} \rightarrow i}^{t+1}\right]_{N}=e^{-\beta h_{N}},} \\
& {\left[\hat{\nu}_{\{i\} \rightarrow i}^{t+1}\right]_{P}=e^{-\beta h_{P}},} \\
& {\left[\hat{\nu}_{\{i\} \rightarrow i}^{t+1}\right]_{U}=e^{-\beta h_{U}} .}
\end{aligned}
$$


Let $\partial_{i}$ denote the set of neighbours of node $i$. The update for $\nu_{i \rightarrow\{i\}}$ is given by

$$
\begin{aligned}
& {\left[\nu_{i \rightarrow\{i\}}^{t+1}\right]_{N}=\prod_{k \in \partial_{i}}\left[\hat{\nu}_{\{k, i\} \rightarrow i}^{t}\right]_{N},} \\
& {\left[\nu_{i \rightarrow\{i\}}^{t+1}\right]_{P}=\prod_{k \in \partial_{i}}\left[\hat{\nu}_{\{k, i\} \rightarrow i}^{t}\right]_{p},} \\
& {\left[\nu_{i \rightarrow\{i\}}^{t+1}\right]_{U}=\prod_{k \in \partial_{i}}\left[\hat{\nu}_{\{k, i\} \rightarrow i}^{t}\right]_{U} .}
\end{aligned}
$$

The update for $\hat{\nu}_{\{i, j\} \rightarrow i}^{t}$ is given by

$$
\begin{aligned}
& {\left[\hat{\nu}_{\{i, j\} \rightarrow i}^{t+1}\right]_{N}=\left[\nu_{j \rightarrow\{i, j\}}^{t}\right]_{N}+e^{-\beta J_{N P}}\left[\nu_{j \rightarrow\{i, j\}}^{t}\right]_{P}+e^{-\beta J_{N U}}\left[\nu_{j \rightarrow\{i, j\}}^{t}\right]_{U},} \\
& {\left[\hat{\nu}_{\{i, j\} \rightarrow i}^{t+1}\right]_{P}=\left[\nu_{j \rightarrow\{i, j\}}^{t}\right]_{P}+e^{-\beta J_{N P}}\left[\nu_{j \rightarrow\{i, j\}}^{t}\right]_{N}+e^{-\beta J_{P U}}\left[\nu_{j \rightarrow\{i, j\}}^{t}\right]_{U},} \\
& {\left[\hat{\nu}_{\{i, j\} \rightarrow i}^{t+1}\right]_{U}=\left[\nu_{j \rightarrow\{i, j\}}^{t}\right]_{U}+e^{-\beta J_{P U}}\left[\nu_{j \rightarrow\{i, j\}}^{t}\right]_{P}+e^{-\beta J_{N U}}\left[\nu_{j \rightarrow\{i, j\}}^{t}\right]_{N} .}
\end{aligned}
$$

The update for $\nu_{i \rightarrow\{i, j\}}$ is given by

$$
\begin{aligned}
& {\left[\nu_{i \rightarrow\{i, j\}}^{t+1}\right]_{N}=e^{-\beta h_{N}} \prod_{k \in \partial_{i} \backslash j}\left[\hat{\nu}_{\{k, i\} \rightarrow i}^{t}\right]_{N},} \\
& {\left[\nu_{i \rightarrow\{i, j\}}^{t+1}\right]_{P}=e^{-\beta h_{P}} \prod_{k \in \partial_{i} \backslash j}\left[\hat{\nu}_{\{k, i\} \rightarrow i}^{t}\right]_{p},} \\
& {\left[\nu_{i \rightarrow\{i, j\}}^{t+1}\right]_{U}=e^{-\beta h_{U}} \prod_{k \in \partial_{i} \backslash j}\left[\hat{\nu}_{\{k, i\} \rightarrow i}^{t}\right]_{U} .}
\end{aligned}
$$

If the BP update equations converge, then $\hat{\nu}_{\{i, j\} \rightarrow i}^{t} \rightarrow \hat{\nu}_{\{i, j\} \rightarrow i}^{*}$ and $\nu_{i \rightarrow\{i, j\}}^{t} \rightarrow \nu_{i \rightarrow\{i, j\}}^{*}$. In that case, one can estimate the marginals for variables nodes via

$$
\begin{aligned}
& {\left[\mu_{i}\right]_{N} \approx e^{-\beta h_{N}} \prod_{k \in \partial_{i}}\left[\hat{\nu}_{\{k, i\} \rightarrow i}^{*}\right]_{N},} \\
& {\left[\mu_{i}\right]_{P} \approx e^{-\beta h_{P}} \prod_{k \in \partial_{i}}\left[\hat{\nu}_{\{k, i\} \rightarrow i}^{*}\right]_{p},} \\
& {\left[\mu_{i}\right]_{U} \approx e^{-\beta h_{U}} \prod_{k \in \partial_{i}}\left[\hat{\nu}_{\{k, i\} \rightarrow i}^{*}\right]_{U} .}
\end{aligned}
$$

For a regular network, one can show that all factor-to-variable messages are identical and all variable-to-factor messages are identical when all messages are initialized 
to the same distribution (e.g., the uniform distribution). Assuming the messages are initialized to a same distribution, the BP equations for a regular network with node degree $k$ are reduced to

$$
\begin{aligned}
& {\left[\hat{\nu}^{t+1}\right]_{N}=\left[\nu^{t}\right]_{N}+e^{-\beta J_{N P}}\left[\nu^{t}\right]_{P}+e^{-\beta J_{N U}}\left[\nu^{t}\right]_{U},} \\
& {\left[\hat{\nu}^{t+1}\right]_{P}=\left[\nu^{t}\right]_{P}+e^{-\beta J_{N P}}\left[\nu^{t}\right]_{N}+e^{-\beta J_{P U}}\left[\nu^{t}\right]_{U},} \\
& {\left[\hat{\nu}^{t+1}\right]_{U}=\left[\nu^{t}\right]_{U}+e^{-\beta J_{P U}}\left[\nu^{t}\right]_{P}+e^{-\beta J_{N U}}\left[\nu^{t}\right]_{N},} \\
& {\left[\nu^{t+1}\right]_{N}=e^{-\beta h_{N}}\left([\hat{\nu}]_{N}^{t}\right)^{k-1},} \\
& {\left[\nu^{t+1}\right]_{P}=e^{-\beta h_{P}}\left([\hat{\nu}]_{p}^{t}\right)^{k-1},} \\
& {\left[\nu^{t+1}\right]_{U}=e^{-\beta h_{U}}\left([\hat{\nu}]_{U}^{t}\right)^{k-1},}
\end{aligned}
$$

where $\nu$ denotes the message from check nodes to variable nodes and $\hat{\nu}$ denotes the message from variable nodes to check nodes.

\subsection{Fixed-Point Belief-Propagation Equations}

In this section, we derive the fixed point BP equations for regular networks. Also, we compute the free entropy for regular networks in terms of the fixed points of BP equations.

Using (4.12), the fixed-point BP equations can be written as

$$
\begin{aligned}
& {[\nu]_{N}=e^{-\beta h_{N}}\left([\nu]_{N}+e^{-\beta J_{N P}}[\nu]_{P}+e^{-\beta J_{N U}}[\nu]_{U}\right)^{k-1}} \\
& {[\nu]_{P}=e^{-\beta h_{P}}\left([\nu]_{P}+e^{-\beta J_{N P}}[\nu]_{N}+e^{-\beta J_{P U}}[\nu]_{U}\right)^{k-1}} \\
& {[\nu]_{U}=e^{-\beta h_{U}}\left([\nu]_{U}+e^{-\beta J_{P U}}[\nu]_{P}+e^{-\beta J_{N U}}[\nu]_{N}\right)^{k-1}}
\end{aligned}
$$

One can estimate the free entropy in terms of the fixed points of the BP messages by computing the Bethe free entropy (Mezard and Montanari, 2009, Section 14.2.4). 
Therefore, we can write

$$
\begin{aligned}
& \Phi(\underline{\nu})=\sum_{\{i, j\} \in A} \log \hat{F}_{\{i, j\}}+\sum_{i=1}^{M} \log \hat{F}_{\{i\}}+\sum_{i=1}^{M} \log F_{i}- \\
& \left(\sum_{\{i, j\} \in A} \log F_{\{i, j\}}+\sum_{i=1}^{M} \log \hat{F}_{i}\right),
\end{aligned}
$$

where $\underline{\nu}$ denotes the set of all fixed point messages in both directions between variable and factor nodes, and

$$
\begin{aligned}
& \hat{F}_{\{i, j\}}=e^{-\beta J_{N P}}\left(\left[\nu_{i \rightarrow\{i, j\}}\right]_{N}\left[\nu_{j \rightarrow\{i, j\}}\right]_{P}+\left[\nu_{i \rightarrow\{i, j\}}\right]_{N}\left[\nu_{j \rightarrow\{i, j\}}\right]_{P}\right)+ \\
& e^{-\beta J_{N U}}\left(\left[\nu_{i \rightarrow\{i, j\}}\right]_{N}\left[\nu_{j \rightarrow\{i, j\}}\right]_{U}+\left[\nu_{i \rightarrow\{i, j\}}\right]_{N}\left[\nu_{j \rightarrow\{i, j\}}\right]_{U}\right)+ \\
& e^{-\beta J_{P U}}\left(\left[\nu_{i \rightarrow\{i, j\}}\right]_{P}\left[\nu_{j \rightarrow\{i, j\}}\right]_{U}+\left[\nu_{i \rightarrow\{i, j\}}\right]_{P}\left[\nu_{j \rightarrow\{i, j\}}\right]_{U}\right)+ \\
& {\left[\nu_{i \rightarrow\{i, j\}}\right]_{N}\left[\nu_{j \rightarrow\{i, j\}}\right]_{N}+\left[\nu_{i \rightarrow\{i, j\}}\right]_{P}\left[\nu_{j \rightarrow\{i, j\}}\right]_{P}+\left[\nu_{i \rightarrow\{i, j\}}\right]_{U}\left[\nu_{j \rightarrow\{i, j\}}\right]_{U},} \\
& \hat{F}_{\{i\}}=e^{-\beta h_{N}}\left[\nu_{i \rightarrow\{i\}}\right]_{N}+e^{-\beta h_{P}}\left[\nu_{i \rightarrow\{i\}}\right]_{P}+e^{-\beta h_{U}}\left[\nu_{i \rightarrow\{i\}}\right]_{U}, \\
& F_{i}=e^{-\beta h_{N}} \prod_{j \in \partial_{i}}\left[\hat{\nu}_{\{i, j\} \rightarrow i}\right]_{N}+e^{-\beta h_{P}} \prod_{j \in \partial_{i}}\left[\hat{\nu}_{\{i, j\} \rightarrow i}\right]_{p}+e^{-\beta h_{U}} \prod_{k \in \partial_{i}}\left[\hat{\nu}_{\{i, j\} \rightarrow i}\right]_{U}, \\
& F_{\{i, j\}}=\left[\nu_{i \rightarrow\{i, j\}}\right]_{N}\left[\hat{\nu}_{\{i, j\} \rightarrow i}\right]_{N}+\left[\nu_{i \rightarrow\{i, j\}}\right]_{P}\left[\hat{\nu}_{\{i, j\} \rightarrow i}\right]_{P}+\left[\nu_{i \rightarrow\{i, j\}}\right]_{U}\left[\hat{\nu}_{\{i, j\} \rightarrow i}\right]_{U} \\
& +\left[\nu_{j \rightarrow\{i, j\}}\right]_{N}\left[\hat{\nu}_{\{i, j\} \rightarrow j}\right]_{N}+\left[\nu_{j \rightarrow\{i, j\}}\right]_{P}\left[\hat{\nu}_{\{i, j\} \rightarrow j}\right]_{P}+\left[\nu_{j \rightarrow\{i, j\}}\right]_{U}\left[\hat{\nu}_{\{i, j\} \rightarrow j}\right]_{U}, \\
& \hat{F}_{i}=e^{-\beta h_{N}}\left[\nu_{i \rightarrow\{i\}}\right]_{N}+e^{-\beta h_{P}}\left[\nu_{i \rightarrow\{i\}}\right]_{P}+e^{-\beta h_{U}}\left[\nu_{i \rightarrow\{i\}}\right]_{U},
\end{aligned}
$$

For a regular network with node degree $k$, assuming all variable to check node messages are initialized to uniform distribution, the equation (4.14) is reduced to

$$
\Phi(\nu, \hat{\nu})=\frac{M K}{2} \log \hat{F}(\nu)+M \log F(\hat{\nu})-\frac{M K}{2} \log \bar{F}(\nu, \hat{\nu}),
$$

where

$$
\begin{aligned}
& \hat{F}(\nu)=[\nu]_{N}^{2}+[\nu]_{P}^{2}+[\nu]_{U}^{2}+ \\
& 2\left([\nu]_{N}[\nu]_{P} e^{-\beta J_{N P}}+[\nu]_{N}[\nu]_{U} e^{-\beta J_{N U}}+[\nu]_{U}[\nu]_{P} e^{-\beta J_{P U}}\right),
\end{aligned}
$$




$$
\begin{gathered}
F(\hat{\nu})=e^{-\beta h_{N}}\left([\hat{\nu}]_{N}\right)^{k}+e^{-\beta h_{P}}\left([\hat{\nu}]_{p}\right)^{k}+e^{-\beta h_{U}}\left([\hat{\nu}]_{U}\right)^{k} \\
\bar{F}(\nu, \hat{\nu})=2\left([\nu]_{N}[\hat{\nu}]_{N}+[\nu]_{P}[\hat{\nu}]_{P}+[\nu]_{U}[\hat{\nu}]_{U}\right)
\end{gathered}
$$

\subsection{Density Evolution for Erdős-Reńyi Random Graphs}

In this section, we analyze the BP algorithm presented in Section 4.3 for random factor graphs determined by Erdős-Reńyi influence graphs. For random factor graphs, messages become random variables. The density evolution approach characterizes the distributions of messages in large random factor graphs (Mezard and Montanari (2009)). To generate a realization of the Erdős-Reńyi graph with $M$ nodes and the parameter $p=c / M$, we start with $M$ disconnected nodes. Then, every two nodes become connected with the probability $p$ (Erdős and Rényi (1959)). It can be shown that for a large Erdős-Reńyi graph, the node degree distribution converges to the Poisson distribution with mean $\lambda=M p=c$.

The density evolution method is based on the assumption that distinct factor nodes in the graph are independent. In our case, one can show that this assumption holds for the random factor graphs corresponding to the Erdős-Reńyi influence networks. In particular, for the factorized representation in Equation (4.5), the factors $\psi_{\{i, j\}}$ and $\psi_{i}$ are independent random variables. Also, for the BP equations in Section 4.3, the density evolution method assumes that the update rule for a variable to a factor node on a given edge depends on that edge only through the node degree of the variable node. Also, it assumes that the updated rule for a factor to variable node on a given edge is independent from that edge.

Let $(i,\{i, j\})$ be a uniformly random edge connecting variable node $i$ to check node $\psi_{\{i, j\}}$ in a given factor graph. The density evolution aims at computing the

distributions of $\nu_{i \rightarrow\{i, j\}}$ and $\hat{\nu}_{\{i, j\} \rightarrow i}$ which contain considerable amount of information regarding the $\mathrm{BP}$ algorithm and its resultant estimated posteriors for the variable 
nodes in the network. Let $B_{i \rightarrow\{i, j\}, t}$ denote the directed neighbourhood of radius $t$ of the directed edge $i \rightarrow\{i, j\}$. Then, Theorem 9.6 in (Mezard and Montanari (2009)) states that $B_{i \rightarrow\{i, j\}, t}$ converges to the random tree $T_{t}$ defined in (Mezard and Montanari, 2009, Section 9.5.1) as $M \rightarrow \infty$. Since $B_{i \rightarrow\{i, j\}, t+1}$ converges in distribution to the tree $T_{t+1}, \nu_{i \rightarrow\{i, j\}}^{t}$ converges in distribution to $\nu^{t}$ as $M \rightarrow \infty$, where $\nu^{t}$ denotes the message passed through the root edge of $T_{t+1}$ after $t$ iterations (Mezard and Montanari, 2009, Proposition 14.9). Therefore, we can write the density evolution equations for the distributions of $\nu^{t}$ and $\hat{\nu}^{t}$ as follows:

$$
\begin{aligned}
& \hat{\nu}^{t+1} \stackrel{d}{=} T\left(\nu^{t}\right), \\
& \nu^{t+1} \stackrel{d}{=} \hat{T}\left(\left\{\hat{\nu}_{k}^{t}\right\}_{1}^{K-1}\right),
\end{aligned}
$$

where $\left\{\hat{\nu}_{k}^{t}\right\}_{1}^{K-1}$ are independent copies of $\hat{\nu}^{t}$ and $K$ is Poisson with mean $\lambda=M p=c$, and

$$
\begin{aligned}
& {\left[T\left(\nu^{t}\right)\right]_{N}=\left[\nu^{t}\right]_{N}+e^{-\beta J_{N P}}\left[\nu^{t}\right]_{P}+e^{-\beta J_{N U}}\left[\nu^{t}\right]_{U},} \\
& {\left[T\left(\nu^{t}\right)\right]_{P}=\left[\nu^{t}\right]_{P}+e^{-\beta J_{N P}}\left[\nu^{t}\right]_{N}+e^{-\beta J_{P U}}\left[\nu^{t}\right]_{U},} \\
& {\left[T\left(\nu^{t}\right)\right]_{U}=\left[\nu^{t}\right]_{U}+e^{-\beta J_{P U}}\left[\nu^{t}\right]_{P}+e^{-\beta J_{N U}}\left[\nu^{t}\right]_{N},} \\
& {\left[\hat{T}\left(\left\{\hat{\nu}_{k}^{t}\right\}_{1}^{K-1}\right)\right]_{N}=e^{-\beta h_{N}} \prod_{k=1}^{K-1}\left[\hat{\nu}_{k}^{t}\right]_{N}} \\
& {\left[\hat{T}\left(\left\{\hat{\nu}_{k}^{t}\right\}_{1}^{K-1}\right)\right]_{P}=e^{-\beta h_{P}} \prod_{k=1}^{K-1}\left[\hat{\nu}_{k}^{t}\right]_{p},} \\
& {\left[\hat{T}\left(\left\{\hat{\nu}_{k}^{t}\right\}_{1}^{K-1}\right)\right]_{U}=e^{-\beta h_{U}} \prod_{k=1}^{K-1}\left[\hat{\nu}_{k}^{t}\right]_{U},}
\end{aligned}
$$

Since the density evolution equations in (4.25) cannot be solved in closed form, we use a numerical method known as population dynamics to estimate the distributions of messages. Population dynamics is a stochastic approach based on the idea of approximating the distributions of $\nu$ and $\hat{\nu}$ using a sample of $L$ i.i.d. copies of $\nu$ : 
Let $\{\nu\}=\left\{\nu_{1}, \nu_{2}, \ldots, \nu_{L}\right\}$ and a sample of $L$ i.i.d copies of $\hat{\nu},\{\hat{\nu}\}=\left\{\hat{\nu}_{1}, \hat{\nu}_{2}, \ldots, \hat{\nu}_{L}\right\}$, respectively, called populations of size $L$ (Mezard and Montanari (2009)). We initialize all the samples in $\{\nu\}$ to $[\nu]_{s}=1 / 3$ for all $s \in\{N, P, U\}$. In each iteration, we first update the samples in $\{\hat{\nu}\}$. Since every factor node $\psi_{\{i, j\}}$ has degree 2 , to update every sample in $\{\hat{\nu}\}$, we randomly choose one sample from $\{\nu\}$. Then, we update the sample in $\{\nu\}$. For every sample in $\{\nu\}$, first, we draw the node degree $K$ from the Poisson distribution with mean $\lambda=M p$. Then, we randomly choose $k-1$ samples from $\{\nu\}$ and update the sample from $\{\nu\}$. This process is repeated for $T$ iterations. Finally, we estimate the node-type marginal distribution using the population $\{\hat{\nu}\}$ and node degrees sampled from the Poisson distribution with mean $\lambda=M p$.

\subsection{Results}

In this section, for regular networks, we first examine whether the fixed point to which the $\mathrm{BP}$ algorithm converges agrees with the stationary distribution computed using the MH algorithm, as described in Section 4.1. Second, we check whether the fixed point, to which the BP algorithm converges, is the solution of the BP fixed point equations in (4.13) and whether maximizes the free entropy in (4.20) among all the solutions of (4.13).

Also, for the Erdős-Reńyi random graphs, first we investigate whether for a given realization of this graph, the average node-type marginal distribution reached by the $\mathrm{BP}$ algorithm agrees with the average node-type marginal of stationary distribution reached by the $\mathrm{MH}$ algorithm. Moreover, we compare the average node-type marginal of distribution estimated using the population dynamics method with the average node-type marginal distribution reached by the BP algorithm over several realizations of the Erdős-Reńyi random graphs.

The MH algorithm is performed on 10 different realizations of random regular networks with $N=1000$ nodes and node degree $k=4$ for 50000 iteration. For each 
graph, we keep running the MH algorithm until the convergence criterion mentioned in Section 4.1 is satisfied. Random regular networks are similar to regular networks in the sense that all nodes have identical node degrees. However, neighbors of each nodes is chosen randomly. The initial percentage of neutral, positive and negative nodes are chosen to be 60, 10 and 30, respectively. In Figure 4.2, we plot the converged node-type marginal stationary distribution in the network versus $J_{N U} / J_{N P}$. To show the mixing behavior of the $\mathrm{MH}$ algorithm, we plot the autocorrelation of samples generated by the $\mathrm{MH}$ algorithm for three different values of $J_{N U} / J_{N P}$ in Figure 4.3. It is observed that the autocorrelation approaches 0 after about 5000 steps. Therefore, running the $\mathrm{MH}$ algorithm for 50000 ensures the mixing of the generated samples. Figure 4.4 shows the estimate of the node-type marginal stationary dis-

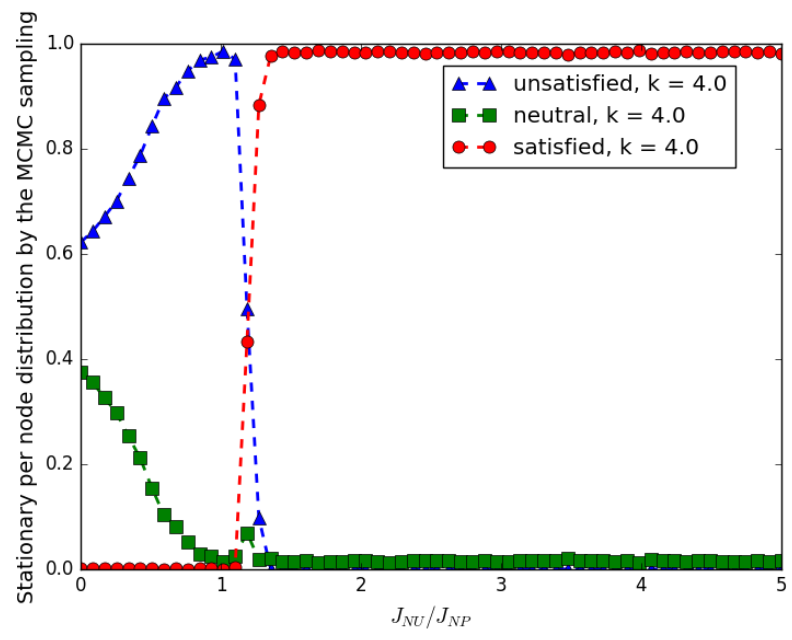

Figure 4.2: Node-type marginal of stationary distribution using the MH algorithm for regular networks with node degree $k=4$ as a function of $J_{N U} / J_{N P}$. Parameter values: $J_{N P}=1.0, J_{P U}=2.0, h_{N}=1.5, h_{P}=1.0, h_{U}=1.0$, and $\beta=1.0$.

tribution given by the $\mathrm{BP}$ algorithm versus $J_{N U} / J_{N P}$ is plotted. We observe a good agreement between the $\mathrm{MH}$ and $\mathrm{BP}$ algorithms.

To investigate whether the estimated node-type marginal distribution using the $\mathrm{BP}$ algorithm is the solution of the fixed point BP equation in (4.13), we perform 


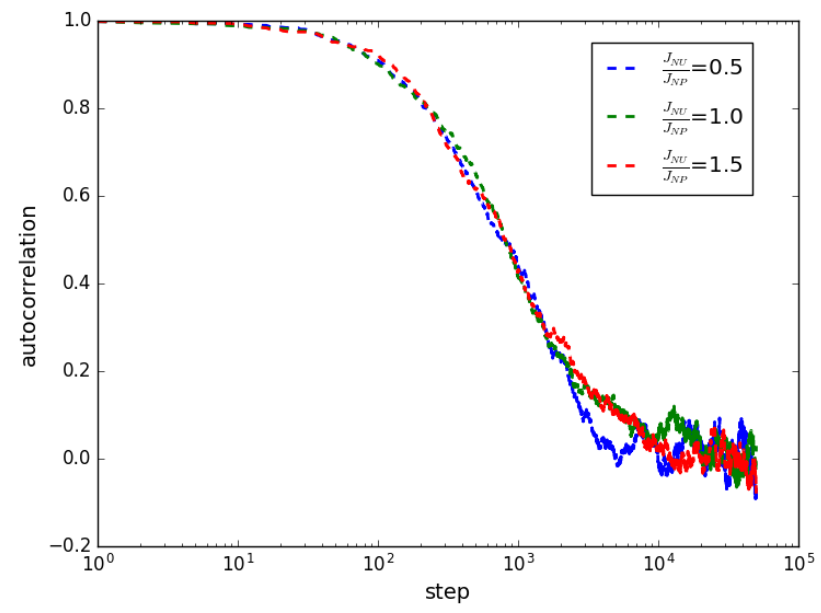

FiguRE 4.3: The sample autocorrelation for the $\mathrm{MH}$ algorithm for regular networks with node degree $k=4$. Parameter values: $J_{N P}=1.0, J_{P U}=2.0, h_{N}=1.5$, $h_{P}=1.0, h_{U}=1.0$, and $\beta=1.0$.

an experiment on regular networks with node degree 4 and the following parameter values: $J_{N P}=1.0, J_{P U}=2.0, J_{N U}=1.0, h_{N}=1.5, h_{P}=1.0, h_{U}=1.0$, and $\beta=1.0$. Running the $\mathrm{BP}$ algorithm for 100 iterations, we observe that all messages converge and in particular the check to variable node messages converge to

$$
[\hat{\nu}]_{N}=0.259 \quad[\hat{\nu}]_{P}=0.097 \quad[\hat{\nu}]_{U}=0.644 .
$$

Then, we numerically solve the fixed point BP equations in (4.13) and find that the message in (4.26) is among the found solutions and it results in the free entropy $\Phi$ of -986.485 . The other solutions are

$$
\begin{aligned}
& {[\hat{\nu}]_{N}=0.436 \quad[\hat{\nu}]_{P}=0.282 \quad[\hat{\nu}]_{U}=0.282,} \\
& \Phi=-1375.429 \text {, } \\
& \begin{array}{c}
{[\hat{\nu}]_{N}=0.0 .318 \begin{array}{c}
{[\hat{\nu}]_{P}=0.341} \\
\Phi=-1375.429
\end{array} \quad[\hat{\nu}]_{U}=0.341,}
\end{array}
\end{aligned}
$$

We find that the fixed point in (4.26), to which the BP algorithm converges, maximizes the Bethe free entropy among the fixed points listed above. 


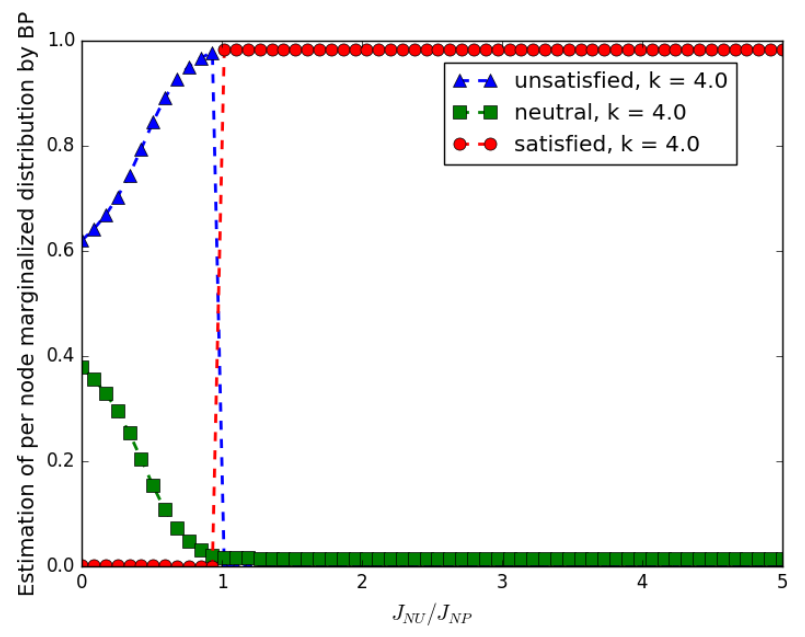

FiguRE 4.4: Estimation of node-type marginal distribution using the BP algorithm for regular networks with node degree $k=4$ as a function of $J_{N U} / J_{N P}$. Parameter values: $J_{N P}=1.0, J_{P U}=2.0, h_{N}=1.5, h_{P}=1.0, h_{U}=1.0$, and $\beta=1.0$.

Next, we validate our results for the Erdős-Reńyi random graphs. First, using the $\mathrm{MH}$ algorithm, we compute the average node-type marginal stationary distribution over 5 realizations of the Erods-Renyi random graphs with $M=1000$ and $p=0.004$. Figure 4.5 (left) plots the computed the average stationary distribution. Then, we compute the average node-type marginal distribution using the BP algorithm for the same realizations of the Erods-Renyi generated to be used for the MH algorithm. Figure 4.5 (right) plots the results for the BP algorithm. The two distributions plotted in Figure 4.5 agree. Finally, we compute the average node-type marginal distribution using the population dynamics method. This distribution is plotted in Figure 4.6 and agrees with the distribution computed using the BP algorithm in Figure 4.5 (right).

\subsection{A Comparison Between Different Scenarios}

In this section, we study different scenarios and examine how modifying the properties of influence networks and the parameters of the Hamiltonian in (4.3) impacts 

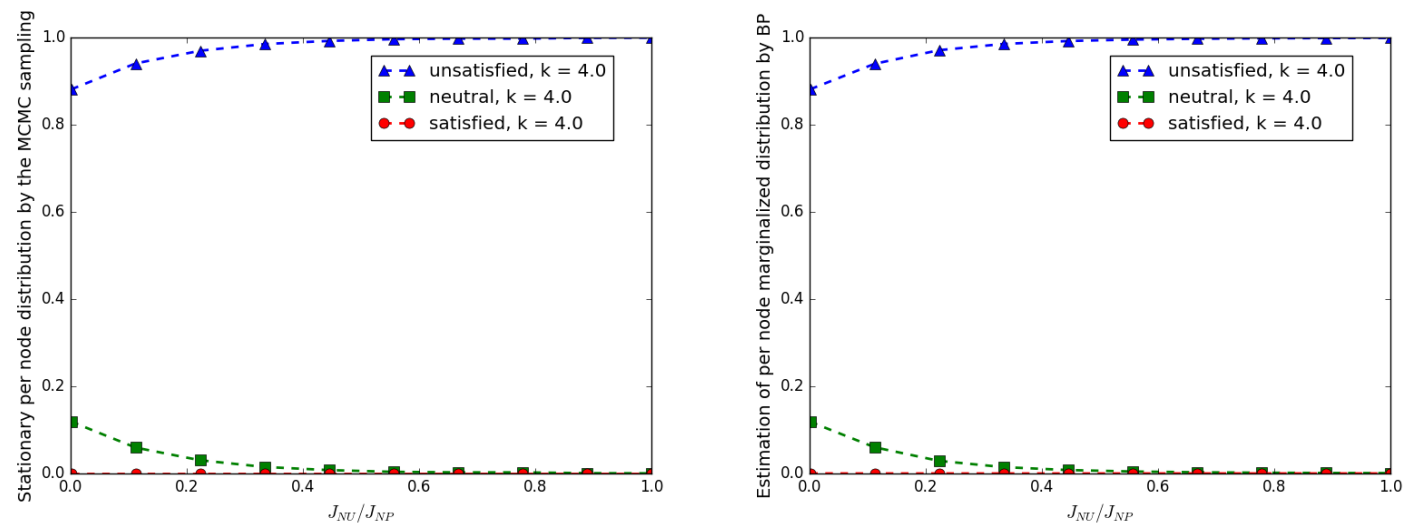

Figure 4.5: (left) Node-type marginal of stationary distribution using the MH algorithm and (right) node-type marginal of stationary distribution using the BP algorithm for Erdős-Reńyi random networks with 1000 nodes and $p=0.004$ as a function of $J_{N U} / J_{N P}$. Parameter values: $J_{N P}=1.0, J_{P U}=3.0, h_{N}=4.0, h_{P}=3.0$, $h_{U}=2.0$, and $\beta=1.0$.

the node-type marginal distribution.

In the first experiment, we examine the impact of increasing the node degree $k$ of regular networks while keeping the parameters of the Hamiltonian in (4.3) fixed. Figures 4.7 and 4.8 depict the node-type marginal distribution using the BP algorithm for regular networks with node degrees 4, 12 and 36. The parameters of the Hamiltonian are: $J_{N P}=1.0, J_{P U}=2.0, h_{N}=1.5, h_{P}=1.0, h_{U}=1.0$, and $\beta=1.0$. In all three cases, we increase $J_{N U}$ while fixing the other parameters. It causes the concentration of the node-type marginal distribution on the positive state. Also, we observe that increasing the node degree delays the transition of the node-type marginal distribution to concentrate on the positive state. This transition happens around $J_{N U} / J_{N P}=1.0,1.5$ and 2.0, respectively, for $k=4,12$ and 36. The significance of $h_{N}, h_{P}$ and $h_{U}$ do not change by changing the node degree since they are independent from the number of neighbours of each nodes and their states. On the other hand, $J_{N P}, J_{N P}$ and $J_{P U}$ model interactions among nodes and their impact on evolution of states of nodes vary by the node degree. 


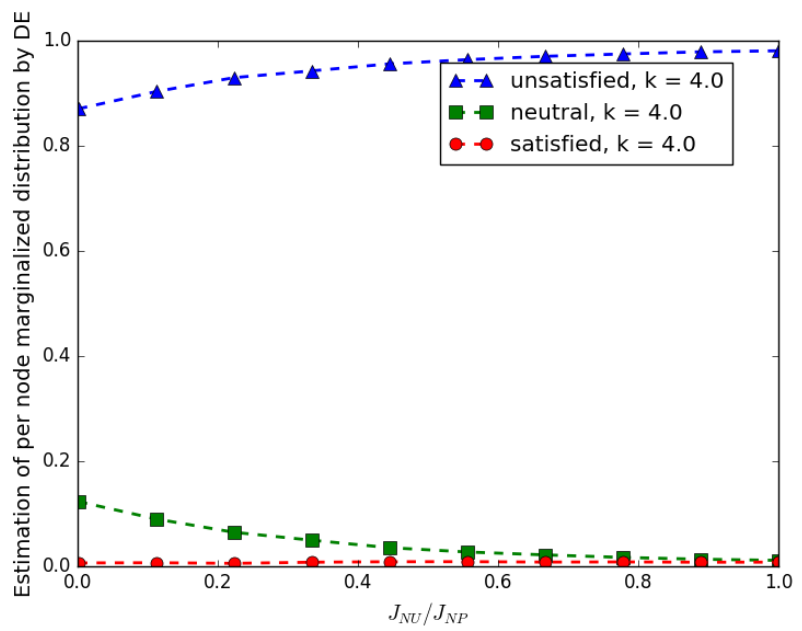

FIGURE 4.6: Estimation of Node-type marginal of stationary distribution using the DE analysis of BP algorithm for Erdős-Reńyi random networks with 1000 nodes and $p=0.004$ as a function of $J_{N U} / J_{N P}$. Parameter values: $J_{N P}=1.0, J_{P U}=3.0$, $h_{N}=4.0, h_{P}=3.0, h_{U}=2.0$, and $\beta=1.0$.

The values of parameters in Figures 4.9 and 4.10 are identical to the above experiment except $J_{P U}=1.0$. In these figures, increasing the node degree does not change the value of $J_{N U} / J_{N P}$ at which the transition of the marginal distribution to concentrate on the positive state happens.

\subsection{Conclusion}

In this chapter, we use a special case of the Potts model to study the propagation of polarized beliefs in social networks. In this adoption model, at any given time $t$, each node is in one of the three states: neutral $(\mathrm{N})$, positive $(\mathrm{P})$ and negative $(\mathrm{U})$. The interactions among individuals are determined by their underlying bidirectional influence networks. We first define the pairwise and individual energy functions to derive the Hamiltonian governing the system.

We use the Metropolis-Hastings algorithm based on the Hamiltonian in Eq. (4.3) to estimate the node-type marginal stationary distribution. Then, we use the BP algorithm to estimate the node-type marginal distribution. For regular networks, we 

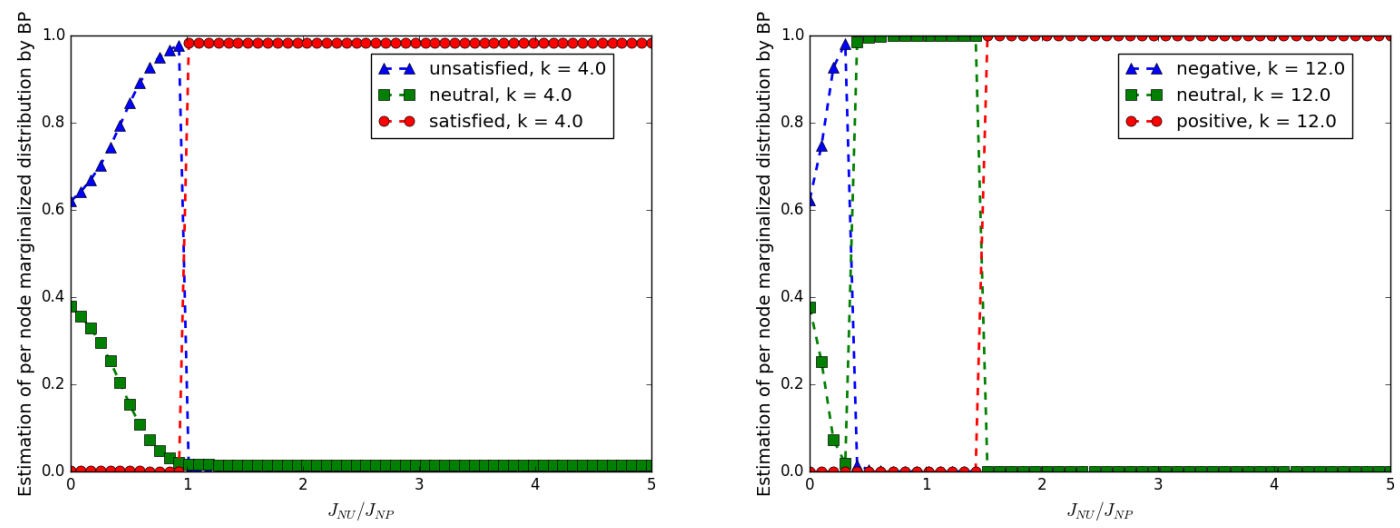

FiguRE 4.7: Estimation of node-type marginal distribution using the BP algorithm for regular networks with node degree $k=4$ (left) and $k=12$ (right) as a function of $J_{N U} / J_{N P}$. Parameter values: $J_{N P}=1.0, J_{P U}=2.0, h_{N}=1.5, h_{P}=1.0, h_{U}=1.0$, and $\beta=1.0$.

simplify the BP equations and derive the fixed-point BP equations. Using the density evolution approach, we analyze the BP algorithm presented in Section 4.3 for random factor graphs determined by Erdős-Reńyi influence graphs. In particular, we use a numerical method known as population dynamics to estimate the distributions of messages. In all cases, we observe that the estimated node-type marginal distribution by the $\mathrm{MH}$ and $\mathrm{BP}$ algorithms agree. 


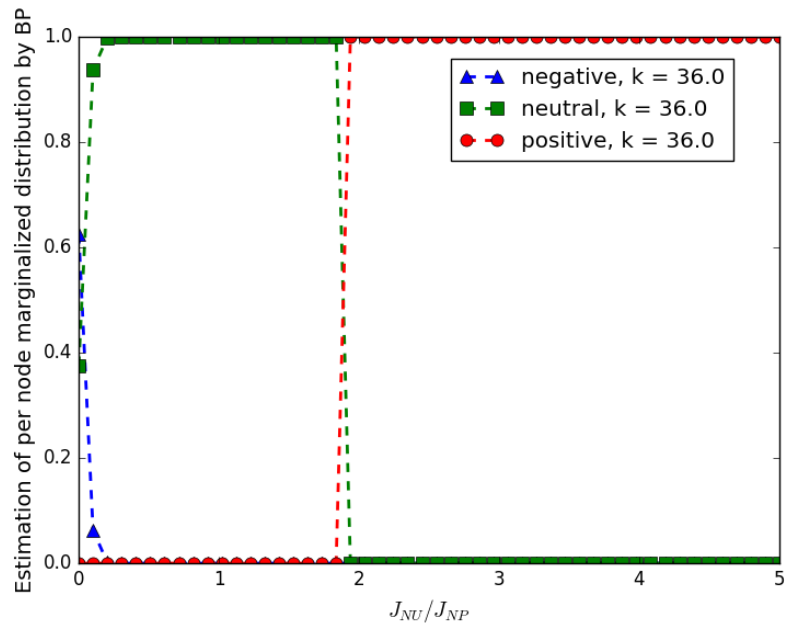

FIGURE 4.8: Estimation of node-type marginal distribution using the BP algorithm for regular networks with node degree $k=36$ as a function of $J_{N U} / J_{N P}$. Parameter values: $J_{N P}=1.0, J_{P U}=2.0, h_{N}=1.5, h_{P}=1.0, h_{U}=1.0$, and $\beta=1.0$.
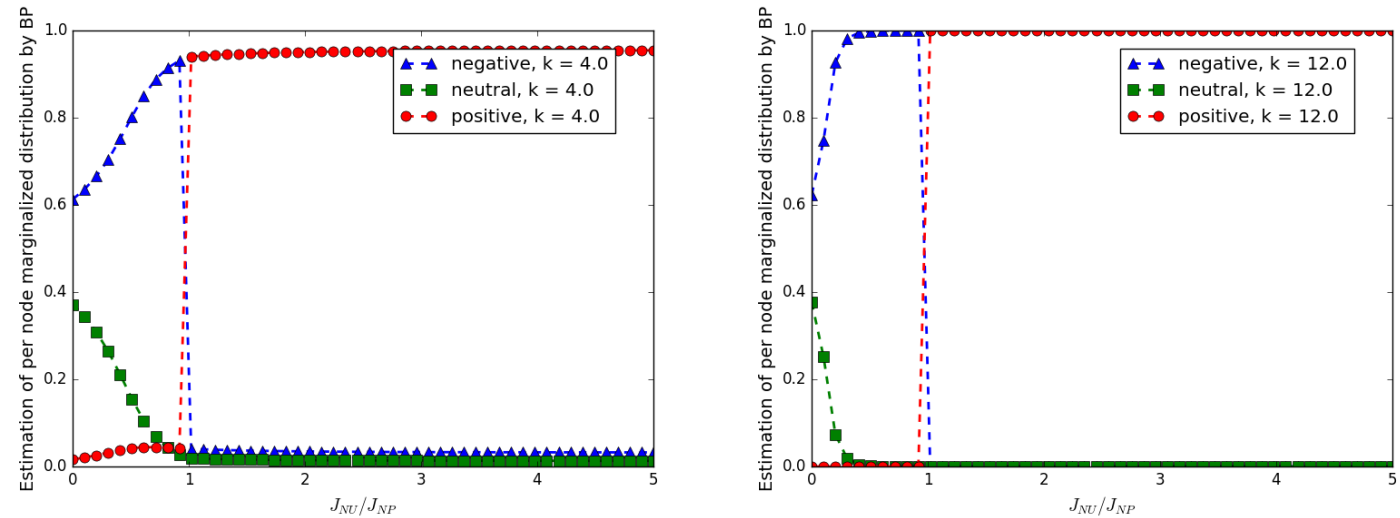

FIGURE 4.9: Estimation of node-type marginal distribution using the BP algorithm for regular networks with node degree $k=4$ (left) and $k=12$ (right) as a function of $J_{N U} / J_{N P}$. Parameter values: $J_{N P}=1.0, J_{P U}=1.0, h_{N}=1.5, h_{P}=1.0, h_{U}=1.0$, and $\beta=1.0$. 


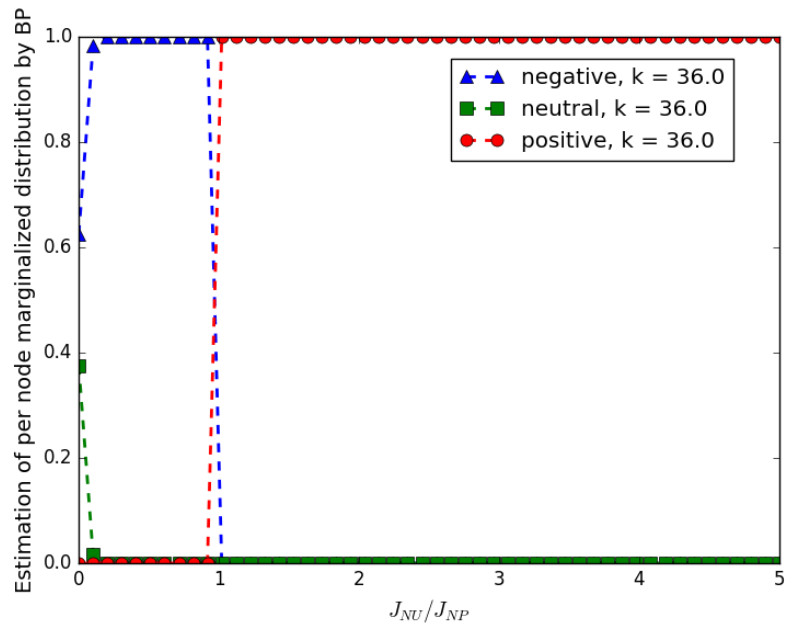

FIGURE 4.10: Estimation of node-type marginal distribution using the BP algorithm for regular networks with node degree $k=36$ as a function of $J_{N U} / J_{N P}$. Parameter values: $J_{N P}=1.0, J_{P U}=1.0, h_{N}=1.5, h_{P}=1.0, h_{U}=1.0$, and $\beta=1.0$. 


\section{Bibliography}

Aral, S. and Walker, D. (2011), "Creating Social Contagion Through Viral Product Design: A Randomized Trial of Peer Influence in Networks," Manage. Sci., 57, 1623-1639.

Aral, S. and Walker, D. (2012), "Identifying Influential and Susceptible Members of Social Networks," Science, 337, 337-341.

Bakshy, E., Hofman, J. M., Mason, W. A., and Watts, D. J. (2011), "Everyone's an Influencer: Quantifying Influence on Twitter," in Proceedings of the Fourth ACM International Conference on Web Search and Data Mining, WSDM '11, pp. 65-74, New York, NY, USA, ACM.

Barabasi, A.-L. and Albert, R. (1999), "Emergence of Scaling in Random Networks," Science, 286, 509-512.

Bogu, M., Pastor-Satorras, R., and Vespignani, A. (2003), "Epidemic Spreading in Complex Networks with Degree Correlations," in Statistical Mechanics of Complex Networks, eds. R. Pastor-Satorras, M. Rubi, and A. Diaz-Guilera, vol. 625 of Lecture Notes in Physics, pp. 127-147, Springer Berlin Heidelberg.

Brock, W. A. and Durlauf, S. N. (2001), "Discrete Choice with Social Interactions," The Review of Economic Studies, 68, pp. 235-260.

Cha, M., Haddadi, H., Benevenuto, F., and Gummadi, K. P. (2010), "Measuring user influence in Twitter: The million follower fallacy," in in ICWSM 10: Proceedings of international AAAI Conference on Weblogs and Social.

Cheng, J., Adamic, L., Dow, P. A., Kleinberg, J. M., and Leskovec, J. (2014), "Can Cascades Be Predicted?" in Proceedings of the 23rd International Conference on World Wide Web, WWW '14, pp. 925-936, New York, NY, USA, ACM.

De Bruyn, A. and Lilien, G. L. (2008), "A multi-stage model of word-of-mouth influence through viral marketing," International Journal of Research in Marketing, $25,151-163$.

Dearing, J. W. (2008), "Evolution of diffusion and dissemination theory." Journal of public health management and practice: JPHMP, 14, 99-108. 
Du, N., Song, L., Gomez-Rodriguez, M., and Zha, H. (2013), "Scalable Influence Estimation in Continuous-Time Diffusion Networks," in Advances in Neural Information Processing Systems 26, eds. C. Burges, L. Bottou, M. Welling, Z. Ghahramani, and K. Weinberger, pp. 3147-3155, Curran Associates, Inc.

Erdős, P. and Rényi, A. (1959), "On Random Graphs I." Publicationes Mathematicae (Debrecen), 6, 290-297.

Farajtabar, M., Du, N., Gomez Rodriguez, M., Valera, I., Zha, H., and Song, L. (2014), "Shaping Social Activity by Incentivizing Users," in Advances in Neural Information Processing Systems 27, Curran Associates, Inc.

Gallager, R. (1963), Low-density Parity-check Codes, M.I.T. Press research monographs, M.I.T. Press.

Goel, S., Watts, D. J., and Goldstein, D. G. (2012), "The Structure of Online Diffusion Networks," in Proceedings of the 13th ACM Conference on Electronic Commerce, EC '12, pp. 623-638, New York, NY, USA, ACM.

Goel, S., Anderson, A., Hofman, J., and Watts, D. (2016), "The structural virality of online diffusion," .

Gomez Rodriguez, M. and Schölkopf, B. (2012), "Influence Maximization in Continuous Time Diffusion Networks," in Proceedings of the 29th International Conference on Machine Learning (ICML 2012), pp. 313-320, New York, NY, USA, Omnipress.

Gomez Rodriguez, M., Leskovec, J., and Krause, A. (2010), "Inferring Networks of Diffusion and Influence," in Proceedings of the 16th ACM SIGKDD International Conference on Knowledge Discovery and Data Mining, KDD '10, pp. 1019-1028, New York, NY, USA, ACM.

Guerra, P. H. C., Jr., W. M., Cardie, C., and Kleinberg, R. (2013), "A Measure of Polarization on Social Media Networks Based on Community Boundaries." in ICWSM, eds. E. Kiciman, N. B. Ellison, B. Hogan, P. Resnick, and I. Soboroff, The AAAI Press.

Hastings, W. K. (1970), "Monte Carlo sampling methods using Markov chains and their applications," Biometrika, 57, 97-109.

Jiménez Felström, A. and Zigangirov, K. S. (1999), "Time-varying periodic convolutional codes with low-density parity-check matrices," IEEE Transactions on Information Theory, 45, 2181-2191.

Jr., D. J. C., Dolecek, L., Fuja, T. E., Kliewer, J., Mitchell, D. G. M., and Smarandache, R. (2013), "Spatially Coupled Sparse Codes on Graphs - Theory and Practice." CoRR, abs/1310.3724. 
Kempe, D., Kleinberg, J., and Tardos, E. (2003), "Maximizing the Spread of Influence Through a Social Network," in Proceedings of the Ninth ACM SIGKDD International Conference on Knowledge Discovery and Data Mining, KDD '03, pp. 137-146, New York, NY, USA, ACM.

Khalil, H. K. (2000), Nonlinear Systems, NJ: Prentice-Hall, third edn.

Kleineberg, K.-K. and Boguñá, M. (2014), "Evolution of the Digital Society Reveals Balance between Viral and Mass Media Influence," Phys. Rev. X, 4, 031046.

Kudekar, S., Richardson, T., and Urbanke, R. (2011), "Threshold Saturation via Spatial Coupling: Why Convolutional LDPC Ensembles Perform So Well over the BEC," IEEE Transactions on Information Theory, 57, 803-834.

Kudekar, S., Richardson, T., and Urbanke, R. (2012), "Spatially coupled ensembles universally achieve capacity under belief propagation," in IEEE International Symposium on Information Theory Proceedings, pp. 453-457.

Kwak, H., Lee, C., Park, H., and Moon, S. (2010), "What is Twitter, a Social Network or a News Media?" in Proceedings of the 19th International Conference on World Wide Web, WWW'10, pp. 591-600, New York, NY, USA, ACM.

Leskovec, J. and Mcauley, J. J. (2012), "Learning to Discover Social Circles in Ego Networks," in Advances in Neural Information Processing Systems 25, eds. F. Pereira, C. Burges, L. Bottou, and K. Weinberger, pp. 539-547, Curran Associates, Inc.

Leskovec, J., Singh, A., and Kleinberg, J. (2006), "Patterns of Influence in a Recommendation Network," in Proceedings of the 10th Pacific-Asia Conference on Advances in Knowledge Discovery and Data Mining, PAKDD'06, pp. 380-389, Berlin, Heidelberg, Springer-Verlag.

Leskovec, J., Adamic, L. A., and Huberman, B. A. (2007), "The Dynamics of Viral Marketing," ACM Trans. Web, 1.

Luby, M. G., Mitzenmacher, M., Shokrollahi, M. A., Spielman, D. A., and Stemann, V. (1997), "Practical Loss-resilient Codes," in Proceedings of the Twenty-ninth Annual ACM Symposium on Theory of Computing, STOC '97, pp. 150-159, New York, NY, USA, ACM.

MacKay, D. J. and Neal, R. M. (1996), "Near Shannon Limit Performance of Low Density Parity Check Codes," Electronics Letters, 32, 1645-1646.

Mezard, M. and Montanari, A. (2009), Information, Physics, and Computation, Oxford University Press, Inc., New York, NY, USA. 
Mieghem, P. V., Omic, J., and Kooij, R. (2009), "Virus spread in networks." IEEE/ACM Trans. Netw., 17, 1-14.

Myers, S., Zhu, C., and Leskovec, J. (2012), "Information Diffusion and External Influence in Networks," .

Pastor-Satorras, R. and Vespignani, A. (2001), "Epidemic dynamics and endemic states in complex networks," Phys. Rev. E, 63, 066117.

Potts, R. B. (1952), "Some generalized order-disorder transformations," Mathematical Proceedings of the Cambridge Philosophical Society, 48, 106-109.

Ribeiro, B. (2014), "Modeling and Predicting the Growth and Death of Membershipbased Websites," in Proceedings of the 23rd International Conference on World Wide Web, WWW'14, pp. 653-664, Republic and Canton of Geneva, Switzerland, International World Wide Web Conferences Steering Committee.

Richardson, T. and Urbanke, R. (2008), Modern Coding Theory, Cambridge University Press, New York, NY, USA.

Richardson, T. J. and Urbanke, R. L. (2002), "The capacity of low-density paritycheck codes under message-passing decoding," IEEE Transactions on Information Theory, 47, 599-618.

Sahneh, F. D., Scoglio, C. M., and Mieghem, P. V. (2013), "Generalized Epidemic Mean-Field Model for Spreading Processes Over Multilayer Complex Networks." IEEE/ACM Trans. Netw., 21, 1609-1620.

Sanatkar, M. R. and Mohammadi, A. (2010), "Scalability Analysis of Wireless Sensor Networks Using Analytical Techniques," in Communication Networks and Services Research Conference (CNSR), 2010 Eighth Annual, pp. 298-303.

Sanatkar, M. R. and Natarajan, B. (2012), "Power selection for maximizing SINR in femtocells with sectorized antennas," in 2012 IEEE Consumer Communications and Networking Conference (CCNC), pp. 690-692.

Sanatkar, M. R. and Pfister, H. D. (2016), "Increasing the rate of spatially-coupled codes via optimized irregular termination," in 2016 9th International Symposium on Turbo Codes and Iterative Information Processing (ISTC), pp. 31-35.

Sanatkar, M. R., Natarajan, B., and Mohammadi, A. (2012), "Route BER estimation in wireless ad hoc networks exploiting minimum distance routing," in 2012 IEEE Consumer Communications and Networking Conference (CCNC), pp. 926-930.

Sanatkar, M. R., Scoglio, C., Natarajan, B., Isard, S. A., and Garrett, K. A. (2015), "History, Epidemic Evolution, and Model Burn-In for a Network of Annual Invasion: Soybean Rust," Phytopathology, 105, 947-955. 
Sanatkar, M. R., White, W. N., Natarajan, B., Scoglio, C. M., and Garrett, K. A. (2016), "Epidemic Threshold of an SIS Model in Dynamic Switching Networks," IEEE Transactions on Systems, Man, and Cybernetics: Systems, 46, 345-355.

Sipser, M. and Spielman, D. A. (1996), "Expander Codes," IEEE Transactions on Information Theory, 42, 1710-1722.

Sun, E., Rosenn, I., Marlow, C., and Lento, T. (2009), "Gesundheit! Modeling Contagion through Facebook News Feed," in International AAAI Conference on Weblogs and Social Media.

Tazoe, K., Kasai, K., and Sakaniwa, K. (2012), "Efficient termination of spatiallycoupled codes," in Information Theory Workshop, pp. 30-34.

Watts, D. J. and Dodds, P. S. (2007), "Influentials, Networks, and Public Opinion Formation," Journal of Consumer Research, 34, pp. 441-458.

Watts, D. J. and Peretti, J. (2007), "Viral Marketing for the Real World," Harvard Business Review.

Watts, D. J. and Strogatz, S. H. (1998), "Collective dynamics of'smallworld'networks." Nature, 393, 409-10.

Yardi, S. and Boyd, D. (2010), "Dynamic Debates: An Analysis of Group Polarization Over Time on Twitter," Bulletin of Science, Technology and Society, 30, $316-327$. 


\section{Biography}

Mohammad Reza Sanatkar was born in 1983 in Mashhad, Iran. He received his B.S. in Electrical Engineering from Ferdowsi University of Mashhad in 2006. He worked as a researcher and software developer from 2006 to 2010 in Nedapardaz Informatic Inc, Tehran, Iran. In Summer 2012, He received his M.S. in Electrical Engineering from Kansas State University. He received his $\mathrm{PhD}$ degree in Computer and Electrical Engineering from Duke University in November 2016.

Publications:

1. Increasing the rate of spatially-coupled codes via optimized irregular termination (Sanatkar and Pfister (2016)).

2. Epidemic threshold of an SIS model in dynamic switching networks (Sanatkar et al. (2016)).

3. History, epidemic evolution, and model burn-in for a network of annual invasion: Soybean rust (Sanatkar et al. (2015)).

4. Route BER estimation in wireless ad hoc networks exploiting minimum distance routing (Sanatkar et al. (2012)).

5. Power selection for maximizing SINR in femtocells with sectorized antennas (Sanatkar and Natarajan (2012)).

6. Scalability Analysis of Wireless Sensor Networks Using Analytical Techniques (Sanatkar and Mohammadi (2010)). 